\title{
Cumulative Bibliography of African Studies: a guide
}

\author{
David Hall
}

The Cumulative Bibliography of African Studies was published in 5 quarto volumes by G.K. Hall of Boston for the International African Institute in 1973.

It attempted to list all the acquisitions of the IAI Library together with volumes in the Library of the School of Oriental and African Studies (SOAS) for the period 1929 to 1970 . It is especially rich in anthropology and linguistics. Much of the material that is ostensibly on other subjects has in fact strong relevance to anthropology.

The Bibliography began as a cumulative card-index of bibliographical entries listed in the International African Institute's journal Africa from 1928 to 1972. Over the years some retrospective items were added. A much longer account of the history is given by Ruth Jones at the beginning of vol. 1 .

3 volumes contain the bibliographical entries arranged according to the major geographical divisions of Africa, subdivided by country and in some cases by region within a country (as is the case with Sudan, Nigeria, Zaire, South Africa). 2 further volumes contain the same entries re-arranged in alphabetical order of author to form an author-catalogue.

Each major geographical division, country and subnational region is allocated a Code-number. So: West-Central Africa is 5, Afrique Equatoriale Française is 51 , Tchad is 511 , République Centrafricaine is 512 , and so on. Within each geopolitical area, entries are arranged by subject (in principle, at least). An elementary index of ethnic groups and languages is given at the end of vol. 3 of the Classified catalogue, referring to code-numbers rather than volume and page-numbers.

Despite the great wealth of its material the Cumulative Bibliography is dauntingly difficult to consult. This is partly because the geographical area of each entry is identified only by its code-number; and even more because the arrangement of subjects is not immediately apparent. Wherever, for any particular area, there is only a small number of entries, they have been subsumed into a larger area. For example, Education in Nigeria or Cameroun is to be found listed under those countries. But Education in The Gambia is subsumed into West Africa along with Guiné Portuguesa and Cape Verde, whereas Education in the Francophone countries of West Africa is subsumed into Afrique Occidentale Française, with any entries for Higher Education being subsumed into West Africa itself.

What follows is an attempt to make this valuable collection more user-friendly. It consists of (1) a synopsis of the Classified Catalogue divided into Africa in general followed by the 7 major regions, with subdivisions into countries; (2) an index of Ethnic Groups; (3) an index of Languages.

The geographical arrangement of the Classified Catalogue is as follows: 
Vol. 1:

1 Africa - General

2 North Africa

3 North-East Africa

Vol. 2:

4 West Africa

5 West Central Africa

Vol. 3:

6 East Africa

7 South-East Central Africa

8 Southern Africa

The 2 volumes of the Author-Catalogue present no problems and are not dealt with here. 


\section{CLASSIFIED CATALOGUE VOLUME 1}

\section{SECTION 1: AFRICA - GENERAL}

Subject-headings followed by an asterisk ${ }^{\star}$ occur only in this section. For other subject-headings (in so far as they occur) see under the geographical area concerned, paying particular attention to the "See also" references at the end of each section

\begin{tabular}{|c|c|c|c|}
\hline \multirow{2}{*}{$\underline{\mathrm{Pag}}$} & \multicolumn{3}{|c|}{ Page } \\
\hline & General & 64 & Broadcasting ${ }^{\star}$ \\
\hline 2 & Administration, government, & 65 & Cannibalism ${ }^{\star}$ \\
\hline & politics & 66 & Ceramics ${ }^{\star}$ \\
\hline 16 & Administration - International & 71 & Chiefs, Kings \\
\hline 18 & Administration - Military (rôle & 73 & Children $^{\star}$ \\
\hline & in politics) ${ }^{\star}$ & 77 & China (and Africa) ${ }^{\star}$ \\
\hline 19 & Adoption ${ }^{\star}$ & & Cinema ${ }^{\star}$ \\
\hline 20 & Aged ${ }^{\star}$ & 78 & Clothing, Adornment ${ }^{\star}$ \\
\hline & Age-grouping ${ }^{\star}$ & 82 & Colours (Significance of) \\
\hline 22 & Agriculture & & Conservation ${ }^{\star}$ \\
\hline 26 & America (and Africa) ${ }^{\star}$ & 84 & Co-operatives ${ }^{\star}$ \\
\hline 27 & Amulets $\star$ & 87 & Cosmology \\
\hline 28 & Ancestors $\star$ & 93 & Cowries $\star$ \\
\hline 29 & Animal-lore ${ }^{\star}$ & & Crafts ${ }^{\star}$ \\
\hline 32 & Anthropology, Applied ${ }^{\star}$ & 95 & Crime $^{\star}$ \\
\hline 34 & Archaeology & 96 & Culture contact ${ }^{\star}$ \\
\hline 36 & Architecture ${ }^{\star}$ & 99 & Currencies (traditional) ${ }^{\star}$ \\
\hline 37 & Art & 101 & Dancing \\
\hline 44 & Asia and Africa ${ }^{\star}$ & 102 & Death \\
\hline & Associations $\star$ & 103 & Demography \\
\hline 45 & Beads, Beadwork ${ }^{\star}$ & 106 & Divination ${ }^{\star}$ \\
\hline 47 & Bee-keeping * & 109 & Divorce* \\
\hline & Belgium & 110 & Dolls* \\
\hline & Bibliographies` & 111 & Drama ${ }^{\star}$ \\
\hline 53 & Birth $^{\star}$ & 114 & Dreams $\star$ \\
\hline 55 & Blood-pacts $\star$ & & Drugs, Alcohol(ism) ${ }^{\star}$ \\
\hline 56 & Botany (Ethno-) ${ }^{\star}$ & 116 & Drums, Drumming $\star$ \\
\hline 59 & Brazil (and Africa) & 119 & Dyeing* \\
\hline 60 & Bride-wealth ${ }^{\star}$ & 120 & Economics, Development \\
\hline 63 & Britain (and Africa) $\star$ & 130 & Education (general) \\
\hline
\end{tabular}


Page

$\begin{array}{ll}137 & \text { Education - Adult } \\ 142 & \text { Education - Agriculture } \\ 143 & \text { Education - Aids } \\ & \text { Education - Arithmetic } \\ & \text { Education - Art, Crafts }\end{array}$

144 Education British policy*

Education - English language*

145 Education - Female ${ }^{\star}$

147 Education - French language*

Education - French policy ${ }^{\star}$

148 Education - Geography ${ }^{\star}$

Education - German policy *

Education - Health

150 Education - Higher

152 Education - History (and Civics)*

153 Education - Indigenous*

155 Education - Italian policy *

Education - Language*

157 Education - Music ${ }^{\star}$

Education - Portuguese policy ${ }^{\star}$

158 Education Science*

Education - Teacher training

159 Education - Vocational ${ }^{\star}$

162 Elites *

164 Ethnicity*

166 Ethnography, Anthropology (Cultural and social)

175 Ethnography - Ethnic groups (in Africa)

Ethnography - Ethnic groups Arabs [see also in vol. 2: Sudan Arab p.568, Ngwana p.602]

176 Ethnography - Ethnic groups Bantu

Ethnography - Ethnic groups Hamitic

177 Ethnography - Ethnic groups Indian

Ethnography - Ethnic groups Jews

Ethnography - Ethnic groups Pygmy (Twide) (see also in vol.

2: Babinga p.484, Pygmy

p.543, Twa p.543, Mbuti p.600, Bayele p.652)

179 Ethnography - Method *

181 Etiquette $^{\star}$

Fertility rites *
Page

182 Fire-making*

183 Fishing

Folklore, Oral literature

186 France (and Africa) ${ }^{\star}$

188 Games *

191 Geography*

192 Germany (and Africa) *

193 History (Oral traditions)

200 Housing

201 Hunting *

204 Indonesia (and Africa)

205 Inheritance ${ }^{\star}$

207 Islam

208 Israel (and Africa) *

Italy (and Africa) ${ }^{\star}$

209 Jewelry*

210 Juvenile delinquency ${ }^{\star}$

211 Kinship, Social structure

213 Labour

218 Labour - Migrant*

222 Land

225 Law

232 Leather-work *

Libraries *

236 Lingua franca

237 Linguistics, Languages

246 Linguistics - Arabic [see also in vol. 1: Sudan Arabic p.572]

Linguistics - Bantu

253 Linguistics - Creole (including

Gulla, Pidgin. See also in vol.

2: Krio p.74; Cape Verde

Islands: Linguistics p.437; São

Tomé and Príncipe: Linguistics p.656)

254 Linguistics, Languages Hamito-Semitic (Afro-Asiatic)

255 Linguistics - Pygmy (see also vol. 2: Twa p.567)

Literature $^{\star}$

262 Livestock $^{\star}$

265 Maps, Marketing *

271 Marriage, Sex

274 Masks *

277 Medicine

281 Metal-working*

287 Migration, Refugees ${ }^{\star}$

290 Missions (Christian)

296 Museums* 


\section{Page}

297 Music

302 Musical Instruments *

307 Mutilation*

308 Names

312 Negro studies $\star$

314 Nomadism $^{\star}$

316 Nutrition

318 Ordeals $^{\star}$

319 Orthography ${ }^{\star}$

322 Periodicals, Press *

324 Personality, Individualism * Poetry ${ }^{\star}$

331 Poisoning ${ }^{\star}$

332 Political systems (Traditional) ${ }^{\star}$

340 Polygamy ${ }^{\star}$

342 Portugal (and Africa) ${ }^{\star}$

343 Property*

344 Psychology

349 Puberty, Initiation *

357 Publishing, Literature provision ${ }^{\star}$

359 Race relations

363 Rain-making *

364 Reckoning, Weights and measures *

369 Religion, magic, philosophy

375 Research (general, inter-disciplinary) ${ }^{\star}$

380 Rock art
Page

381 Rural studies*

382 Russia (and Africa)*

384 Sacrifice $^{\star}$

385 Secret societies

390 Separatist movements

391 Slavery, slave trade*

397 Social change

402 Social stratification *

405 Social welfare

406 Songs, Singing ${ }^{\star}$

412 Spain (and Africa)

413 Suicide*

Switzerland (and Africa) *

Taboo*

414 Tattooing, Body-painting, etc. *

416 Textiles, Weaving, etc. ${ }^{\star}$

419 Tone (in African languages) ${ }^{\star}$

423 Toponyms*

428 Totemism*

429 Transport (Traditional) ${ }^{\star}$

431 Twins*

432 Urban studies

435 Warfare. (Traditional) *

436 Weapons

439 West Indies (and Africa) ${ }^{\star}$

440 Witchcraft ${ }^{\star}$

446 Women

448 Writing (Scripts) *

449 Youth* 


\section{SECTION 2: NORTH AFRICA}

The arrangement of North Africa is as follows:

NORTH AFRICA

MOROCCO

IFNI

THE RIF

CANARIES

ALGERIA

TUNISIA

LIBYA

EGYPT

\section{NORTH AFRICA}

\section{Code-number 2}

\section{NORTH AFRICA}

451 Unclassified

452 Administration (including

Egypt. See also in vol. 1:

Morocco p.484, Algeria p.497,

Tunisia p.511 and Libya p.517)

Agriculture (including Libya and Egypt. See also: Morocco p.485, Algeria p.498 and

Tunisia p.511)

453 Archaeology (including all countries of North Africa)

455 Art (including all countries of North Africa)

456 Death (Including all countries of North Africa)

Demography (Including

Morocco, Libya and Egypt. See also Algeria p.498 and Tunisia p.512)

457 Economics (including Libya and Egypt. See also Morocco p.486, Algeria p.499 and Tunisia p.513)

459 Education (including all countries of North Africa)

460 Ethnography (See also Ethnography in vol. 1 as follows: Arabs p.175, Morocco p.486, Ifni p.494, The Rif p.495, Canaries p.496, Algeria

$\frac{\text { Page }}{451}$
483
494
494
496
497
510
516
521

p.500, Tunisia p.514, Libya

p.518, Egypt p.521)

- Unclassified ethnography

461 - Berber (See also in vol. 1:

Berber p.488, p.502, p.519)

Family. See below: Marriage,

Family

462 Folklore (including all countries of North Africa)

464 History (including Algeria, Tunisia, Libya and Egypt. See also Morocco p.492)

466 Housing (including all countries of North Africa)

467 Islam (including all countries of North Africa)

468 Kinship (including all countries of North Africa)

Labour (including all countries of North Africa)

469 Land (including all countries of North Africa)

470 Law (including all countries of North Africa)

472 Linguistics (See also in vol. 1:

Arabic p.246. See also

Linguistics in vol. 1 as follows:

Morocco p.492, The Rif p.495,

Canaries p.496, Algeria p.508, Tunisia p.515, Libya p.520,

Egypt p.523)

- Unclassified linguistics

- Berber (See also in vol. 1:

Berber p.493, p.496, p.508, p.515, p.520) 
475 Marriage \& Family (including all countries of North Africa)

476 Medicine (including all countries of North Africa)

477 Missions (Christian) (including all countries of North Africa) Music (including all countries of North Africa)

Nutrition (including all countries of North Africa)

478 Psychology (including all countries of North Africa)

Religion (including all countries of North Africa)

479 Rock art (including all countries of North Africa)

482 Urban studies (including all countries of North Africa) Women (including all countries of North Africa)

For other subjects, see the asterisked headings in Vol. 1, Section 1

\section{MOROCCO}

\section{Code Number 21}

\section{MOROCCO}

\section{Unclassified}

484 Administration (including Ifni and the Rif)

485 Agriculture

486 Economics

Ethnography

- Unclassified ethnography

488 - Berber (See also in vol. 1:

Berber p.461, p.495)

492 History

Linguistics

- Unclassified linguistics

493 - Berber (See also in vol. 1:

Berber p.472, p.496)

Any relevant entries on the following subjects will be found subsumed into NORTH AFRICA in volume 1: Unclassified p.451, Archaeology p.453, Art p.455, Death p.456,
Demography p.456, Education p.459, Folklore p.462, Housing p.466, Islam p.467, Kinship p.468, Labour p.468, Land p.469, Law p.470, Marriage \& Family p.475, Medicine p.476, Missions (Christian) p.477, Music p.477, Nutrition p.477, Psychology p.478, Religion p.478, Rock art p.479, Urban studies p.482, Women p.482

For other possible subjects, see the asterisked headings in Vol. 1, Section 1

See also in vol. 1: Spanish Morocco (Rif) p.494 seq.; in vol. 2: Spanish Sahara (Rio de Oro) p.438

\section{IFNI}

\section{Code-Number 211}

494 IFNI

Unclassified

Ethnography

- Unclassified ethnography

- Ait Baamran

For other possible subjects, see the asterisked headings in Vol. 1, Section 1

\section{RIF}

\section{Code-Number 212}

494 RIF

Unclassified

495 Ethnography

- Unclassified ethnography

- Berber

Linguistics

- Unclassified linguistics

496 - Berber

For other possible subjects, see the asterisked headings in Vol. 1, Section 1 


\section{CANARIES}

\section{Code-Number 213}

496 CANARIES

Unclassified

Ethnography

- Unclassified ethnography

Linguistics

Unclassified Linguistics

For other possible subjects, see the asterisked headings in Vol. 1, Section 1

\section{ALGERIA}

\section{Code-Number 22}

497 ALGERIA

Unclassified

Administration

498 Agriculture

Demography

499 Economics

500 Ethnography

- Unclassified ethnography

$502-$ Berber (See also in vol. 1:

Berber p.461)

503 - Kabyle

504 - Mzab

505 - Shawiya;. Tuareg

508 Linguistics

- Unclassified linguistics

- Berber (See also in vol. 1:

Berber p.472)

509 - Kabyle; Shawiya

510 -Tuareg

Any relevant entries on the following subjects will be found subsumed into NORTH AFRICA in volume 1: Unclassified p.451, Archaeology p.453, Art p.455 Death p.456, Education p.459, Folklore p.462, History p.464, Housing p.466, Islam p.467, Kinship p.468, Labour p.468, Land p.469, Law p.470, Marriage \& Family p.475, Medicine p.476, Missions (Christian) p.477, Music p.477, Nutrition p.477,
Psychology p.478, Religion p.478, Rock art p.479, Urban studies p.482, Women p.482

For other possible subjects, see the asterisked headings in Vol. 1, Section 1

\section{TUNISIA}

\section{Code-Number 23}

510 TUNISIA

Unclassified

511 Administration

Agriculture

512 Demography

513 Economics

514 Ethnography

- Unclassified ethnography

515 Linguistics

- Unclassified linguistics

- Berber (See also in vol. 1:

Berber p.472)

Any relevant entries on the following subjects will be found subsumed into NORTH AFRICA in volume 1: Unclassified p.451, Archaeology p.453, Art p.455, Death p.456, Education p.459, Folklore p.462, Housing p.466, Islam p.467, Kinship p.468, Labour p.468, Land p.469, Law p.470, Marriage \& Family p.475, Medicine p.476, Missions (Christian) p.477, Music p.477, Nutrition p.477, Psychology p.478, Religion p.478, Rock art p.479, Urban studies p.482, Women p.482

For other possible subjects, see the asterisked headings in Vol. 1, Section 1

\section{LIBYA}

\section{Code-Number 24}

\section{LIBYA}

Unclassified 
517 Administration

518 Ethnography (See also in vol. 2: Teda p.494)

- Unclassified ethnography

519 - Berber (See also in vol. 1: Berber p.461)

520 - Garamantes

Linguistics

- Unclassified linguistics

- Berber (See also in vol. 1:

Berber p.472)

Any relevant entries on the following subjects will be found subsumed into NORTH AFRICA in volume 1: Unclassified p.451, Agriculture p.452, Archaeology p.453, Art p.455, Death p.456, Demography p.456, Economics p.457, Education p.459, Folklore p.462, History p.464, Housing p.466, Islam p.461, Kinship p.468, Labour p.468, Land p.469, Law p.470, Marriage \& Family p.475, Medicine p.476, Missions (Christian) p.417, Music p.477, Nutrition p.477, Psychology p.478, Religion p.478, Rock art p.4.79, Urban studies p.482, Women p.482

For other possible subjects, see the asterisked headings in Vol. 1, Section 1

\section{EGYPT}

\section{Code-Number 25}

521 EGYPT

Unclassified

Ethnography

- Unclassified ethnography

522 - Siwa

523 Linguistics

- Unclassified linguistics

- Siwi

Any relevant entries on the following subjects will be found subsumed into NORTH AFRICA in volume 1: Unclassified p.451, Administration p.452, Agriculture p.452, Archaeology p.453, Art p.455, Death p.456, Demography p.456, Economics p.457, Education p.459, Folklore p.462, History p.464, Housing p.466, Islam p.467, Kinship p.468, Labour p.468, Land p.469, Law p.470, Marriage \& Family p.475, Medicine p.476, Missions (Christian) p.477, Music p.477, Nutrition p.477, Psychology p.478, Religion p.478, Rock art p,479, Urban studies p.482, Women p.482

For other possible subjects, see the asterisked headings in Vol. 1, Section 1 


\section{SECTION 3: NORTH-EAST AFRICA}

\section{Code Number 3}

The arrangement of North-East Africa is as follows:

NORTH-EAST AFRICA

SUDAN

ETHIOPIA

ERITREA

SOMALIA

\section{Page \\ 524 \\ 548 \\ 593 \\ 631 \\ 635}

\section{NORTH-EAST AFRICA}

\section{NORTH-EAST AFRICA \\ Unclassified \\ Administration (including}

Eritrea. See also in vol. 1:

Sudan p.549, Ethiopia p.596,

Somalia p.635)

Agriculture (including

Ethiopia, Eritrea and Somalia.

See also in vol. 1: Sudan p.551)

525 Archaeology (including

Ethiopia, Eritrea and Somalia.

See also in vol. 1: Sudan p.552)

527 Art (including Sudan, Ethiopia,

Eritrea and Somalia)

529 Chiefs (including Sudan,

Ethiopia, Eritrea and Somalia)

530 Dancing (including Sudan,

Ethiopia, Eritrea and Somalia)

Death (including Sudan,

Ethiopia, Eritrea and Somalia)

531 Demography (including Sudan, Ethiopia, Eritrea and Somalia)

532 Economics (including Ethiopia, Eritrea and Somalia. See also in vol. 1: Sudan p.554)

534 Education, (including Ethiopia, Eritrea and Somalia. See also in vol. 1: Sudan p.556)

535 Ethnography (see also in vol. 1: Sudan p.557, Ethiopia p.598, Eritrea p.631 and Somalia p.631)

- Unclassified ethnography Folklore (including Sudan, Eritrea and Somalia. See also in vol. 1: Ethiopia p.610)
536 History (including Eritrea and Somalia. See also in vol. 1 : Sudan p.558, Ethiopia p.611)

537 Housing (including Sudan, Ethiopia, Eritrea and Somalia) Islam (including Sudan, Ethiopia, Eritrea and Somalia)

538 Kinship (including Sudan, Ethiopia, Eritrea and Somalia)

539 Labour (including Sudan, Ethiopia, Eritrea and Somalia) Land (including Sudan, Ethiopia, Eritrea and Somalia)

540 Law (including Ethiopia, Eritrea and Somalia. See also in vol. 1: Sudan p.560)

543 Linguistics (see also in vol. 1: Sudan p.560, Ethiopia p.616, Eritrea p.633 and Somalia p.638)

- Unclassified linguistics Marriage (including Sudan, Ethiopia, Eritrea and Somalia)

544 Medicine (including Sudan, Ethiopia, Eritrea and Somalia)

545 Missions, Christian (including Sudan, Ethiopia, Eritrea and Somalia)

546 Music (including Sudan, Ethiopia, Eritrea and Somalia) Nutrition (including Sudan, Ethiopia, Eritrea and Somalia)

547 Religion (including Eritrea and Somalia. See also in vol. 1: Sudan p.561, Ethiopia p.628) Rock art including Sudan, Ethiopia, Eritrea and Somalia) 548 Urban studies (including 
Sudan, Ethiopia, Eritrea and Somalia)

Women (including Sudan, Ethiopia, Eritrea and Somalia)

For other possible subjects, see the asterisked headings in Vol. 1, Section 1

\section{SUDAN}

\section{Code-Number 31}

\section{SUDAN, GENERAL}

548 SUDAN, GENERAL (See also in vol. 1: Sudan, Northern p.562; Sudan, Southern p.572) Unclassified

549 Administration (including Northern and Southern Sudan)

551 Agriculture (including Northern and Southern Sudan)

552 Archaeology (including Northern and Southern Sudan)

554 Economics (including Northern and Southern Sudan)

556 Education (including Northern and Southern Sudan)

557 Ethnography (See also in vol. 1: Sudan, Northern p.563; Sudan, Southern p.573)

- Unclassified ethnography

558 History. (including Northern and Southern Sudan)

560 Law (including Northern and Southern Sudan)

Linguistics (See also in vol. 1:

Sudan, Northern p.570; Sudan, Southern $\mathrm{p}, 589$ )

- Unclassified linguistics

561 Religion (including Northern and Southern Sudan)

Any relevant entries on the following subjects will be found subsumed into NORTH EAST AFRICA in volume 1: Unclassified p.524, Art p.527, Chiefs p.529, Dancing p.530, Death p.530, Demography p.5.31, Folklore p.535, Housing p.537, Islam p.537, Kinship p.538, Labour p.539, Land p.539, Marriage p.543, Medicine p.514, Missions, Christian p.545, Music p.546, Nutrition p.546, Rock art p.547, Urban studies p.548, Women p.548

For other possible subjects, see the asterisked headings in Vol. 1, Section 1

\section{SUDAN, NORTHERN}

\section{Code-number 311}

\section{SUDAN, NORTHERN}

Unclassified

563 Ethnography - (see also in vol. 1: Fung p.578, Ingassana p.578; see also in vol. 2:

Zaghawa p.495)

- Unclassified ethnography

- Beja (including Ababda, Amarar, Ashraf, Beni Amer, Bisharin, Hadendowa, Halenga

565 - Berti; Dago (Dagu); Fur; Meroitic; Nubian (including Barabra, Hawawir, Mahas, Midob)

568 - Sudan Arab (including Abdullab, Abudab, Baggara, Batahin, Gamuia, Halawin, Hamran, Homr or Humr, Kababish, Khawalda, Mamluk, Messiria, Rubatab, Shaikiya, Shukriya)

570 - Takruri (Tekruri) Linguistics (Ingassana, see also in vol. 1: Ingassana p.591; see also in vol. 2: Tama p.497)

- Unclassified linguistics

- Bedawiye (Bedauye, Beja)

(including Bisharin, Hadendowa); Berti; Fur (Konjara)

571 - Kara; Meroitic; Nubian (including Dongolawi, Birged, 
Kenuz or Kunuz, Mahas, Midob; see also in vol. 1: Nubian p.592)

572 - Sudan Arabic; Toposa

For other possible subjects, see the asterisked headings in Vol. 1, Section 1

\section{SUDAN, SOUTHERN}

\section{Code-number 312}

\section{SUDAN, SOUTHERN}

Unclassified

573 Ethnography See also in vol. 1:

Berta p.601, Dago p.565, Fur p.565, Koma p.608, Surma

p.610; see also in vol. 2: Banda

p.498, Logo-Avokaya p.598; see also in vol. 3: Acoli p.134,

Nilo-Hamitic p.22)

- Unclassified ethnography

574 - Anuak (Yambo) (including

Pari or Beri or Föri or Lokoro)

575 - Bandala; Bari (including Nyangbara); Bongo-Baka (group) (including Morokodu, Yulu, Kara)

576 - Burun (including Maban);

Didinga; Dinka

578 - Feroge (group) (including Indri, Mangaya, Togoyo); Fung; Gob; Ingassana (Tabi);

Kakwa; Kresh (Gbaya) (including Aja, Dongo, Woro)

579 - Kuku; Lango (including Dongotono); Longarim;

Lotuko (Latuka, Lotuxo); Lwo (group) (including Belanda or Bor or Mberidi, Jur or Shilluk Luo, Manangeer)

580 - Mondari (Mandari); Moru-Madi (group) (including Metu, Nyonose)

581 - Murle (Beir); Ndogo-Sere (group) (including Bai, Belanda or Bviri or Mbegumba); Ngala
(Ngara); Nilotic (group)

582 - Nuba (including Afitti or

Dair, Dilling, Dubab, 'Hill'

Nubian, Nyamang, Nyaro,

Tegalawi, Tulishi

583 - Nuer

585 - Shilluk

586 - Suri; Toposa; Zande

(Azande, Avungara,

Nyam-Nyam) (See also in vol.

2: Zande p.602)

589 Linguistics (see also in vol. 1:

Fur p.570, Koma p.627; see

also in vol. 2: Logo-Avokaya

p.605; see also in vol. 3 :

Nilo-Hamitic p.40; Acoli

p.155)

589 - Unclassified linguistics

590 - Anuak; Bari (including

Kakwa, Mundari, Nyangbara);

Bongo-Baka (including Yulu);

Didinga; Dinka (Denka, Jieng)

(including Bon, Rek)

591 - Feroge (including Indri,

Mangaya, Togoyo); Golo;

Ingassana (Tabi); Koalib-Tagoi

(group) (including Heiban,

Tumale); Kresh (Gbaya)

(including Binga); Lotuko

(Latuka, Lotuxo) (including

Oxoriok); Lwo (Jur);

Moru-Madi (group)

592 - Murle (Beir); Ndogo

(including Birri or Bviri); Ngala

(Ngara); Nilotic (group);

Nubian ('Hill' Nubian)

(including Dair, Delen or

Dilling); Nuer

593 - Shilluk; Zande (Azande, Nyam-Nyam) (see also in vol.

2: Zande p.605)

For other possible subjects, see the asterisked headings in Vol. 1, Section 1 


\section{ETHIOPIA}

Code-number 32. (Includes works jointly on Ethiopia and Eritrea. For works on Eritrea alone see vol. 1: Eritrea, p.631 seq.)

\section{ETHIOPIA \\ Unclassified}

596 Administration

598 Ethnography (See also in vol. 1: Afar p.632, Anuak p.574, Fung p.578, Saho (including Irob)

p.633, Somali p.637, Suri

p.586)

- Unclassified ethnography

601 - Berta (Beni Shangul); Burji-Konso (group) (including Amar, Arbore, Geleba)

602 - Dime; Dukunz; Ethiopians

(Abyssinians, Amhara)

603 - Falasha

604 - Gafat; Galla (Oromo) (including Arussi, Boran)

607 - Gimira-Maji (group)

(Ghimirra, Magi); Gumuz

(Gumus, Gunza); Gurage

(including Ennemor, Chaha, Galila)

608 - Hadiya; Harari (inhabitants of

Harar city); Janjero; Kafa

(Kaffa, Kaffitsho, Gonga);

Kemant (Qemant); Koma

(including Uduk)

609 - Mao; Masongo (Majangir,

Ujang); Shinasha; Sidama

(including Darassa, Ometo,

Sidamo, Wolamo, Zala)

610 - Surma (group) (including

Tid, Tirma, Zelmamu)

Folklore

\section{History}

616 Linguistics. (see also in vol. 1:

Afar p.633, Anuak p.590,

Somali p.639)

- Unclassified linguistics

619 - Agau (group) (including

Awiya, Kemant, Khamir,

Kwara or Quara; see also in vol.
1: Bilin p.633); Amharic

(Amarinya)

622 - Argobba

623 - Baiso; Berta (including

Fazoglo, Gamila); Gaflit; Galla (Oromo) (including Boran)

624 - Ge'ez (Ghez, classical

Ethiopic)

625 - Gimira-Maji (group)

(including Nao); Gurage

(including Chaha)

626 - Hadiya; Harari; Janjero

627 - Kafa (Kaffa, Caffino)

(including Mocha); Koma

(including Gumuz);

Konso-Geleba (group)

(including Arbore, Bambala or

Burji, Gidole, Gowaze);

Masongo; Mekan (including

Tirma); Ometo (group)

(including Haruro, Wolamo);

Sidamo (E. Sidama), group

(including Alaba, Darasa,

Kambatta)

628 - Tigrinya (Tigrai)

Religion

Any relevant entries on the following subjects will be found subsumed into NORTH EAST AFRICA in volume 1: Unclassified p.524, Agriculture p.524, Archaeology p.525, Art p.527, Chiefs p.529, Dancing p.530, Death p.530, Demography p.531, Economics p.532, Education p.534, Housing p.537, Islam p.537, Kinship p.538, Labour p.539, Land p.539, Law p.540, Marriage p.543, Medicine p.544, Missions, Christian p.545, Music p.546, Nutrition p.546, Rock art p.547, Urban studies p.548, Women p.548

For other possible subjects, see the asterisked headings in Vol. 1, Section 1 


\section{ERITREA}

Code-number 33. (See also in vol. 1: Ethiopia p.593 seq. for works jointly on Ethiopia and Eritrea)

\section{ERITREA}

Unclassified

Ethnography (see also in vol. 1:

Beja p.563, Takruri p.570)

- Unclassified ethnography

632 - Afar (Danakil); Bulin

(Bogos); Habab; Kunama (and Baria)

633 - Mensa; Saho (including Assaorta)

Linguistic

- Unclassified linguistics

- Afar (Danakil); Baria (Barea);

Bilin; Kunama

634 - Saho (including Irob); Tigre

Any relevant entries on the following subjects will be found subsumed into NORTH EAST AFRICA in volume 1: Unclassified p.524, Administration p.524, Agriculture p.524, Archaeology p.525, Art p.527, Chiefs p.529, Dancing p.530, Death p.530, Demography p.53 1, Economics p.532, Education p.534, Folklore p.535, History p.536, Housing p.537, Islam p.537, Kinship p.538, Labour p.539, Land p.539, Law p.540, Marriage p.543, Medicine p.544, Missions, Christian p.545, Music p.546, Nutrition p.546, Religion p.547, Rock art p.547, Urban studies p.548, Women p.548

For other possible subjects, see the asterisked headings in Vol. 1, Section 1

\section{SOMALIA}

635 SOMALIA (and Territoire Français des Afars et des Issas) Code-number 34
635 SOMALIA (See also in vol. 1: Somaliland p.641, Territoire Français des Afars et des Issas p.642, Somalia (ex-Italian) p.643. For works jointly on Ethiopia and the Horn, see in vol. 1: Ethiopia p.593 seq.) Unclassified Administration (including ex-British Somaliland, Territoire Français des Afars et des Issas, ex-Italian Somalia)

637 Ethnography

- Unclassified ethnography

- Somali

638 Linguistics

- Unclassified linguistics

639 - Somali

Any relevant entries on the following subjects will be found subsumed into NORTH EAST AFRICA in volume 1: Unclassified p.524, Agriculture p.524, Archaeology p.525, Art p.527, Chiefs p.529, Dancing p.530, Death p.530, Demography p.53 1, Economics p.532, Education p.534, Folklore p.535, History p.536, Housing p.537, Islam p.537, Kinship p.538, Labour p.539, Land p.539, Law p.540, Marriage p.543, Medicine p.544, Missions, Christian p.545, Music p.546, Nutrition p.546, Religion p.547, Rock art p.547, Urban studies p.548, Women p. 548

For other possible subjects, see the asterisked headings in Vol. 1, Section 1

\section{SOMALILAND (ex-British)}

\section{Code-number 341}

\section{SOMALILAND (ex-British)}

642 Ethnography (see also in vol. 1: Somali p.637)

For other possible subjects, see the 
asterisked headings in Vol. 1, Section 1

\section{TERRITOIRE FRANCAIS DES AFARS ET DES ISSAS, EX-COTE FRANCAISE DES SOMALIS}

\section{Code-number 342}

642 TERRITOIRE FRANÇAIS DES AFARS ET DES ISSAS, EX-COTE FRANÇAISE DES SOMALIS

Unclassified

Ethnography (See also in vol. 1:

Somali p.637, Afar p.632.)

- Unclassified ethnography

For other possible subjects, see the asterisked headings in Vol. 1, Section 1

\section{SOMALIA (ex-Italian)}

\section{Code-number 343}

643 SOMALIA (ex-Italian). (See also in vol. 1: Somalia p.635 seq.) Unclassified
Ethnography (See also in vol. 1: Somali p.637)

- Unclassified ethnography

644 - Bajuni; Boni (Waboni)

Linguistics (See also in vol. 1:

Linguistics p.638 seq.)

- Unclassified linguistics

- Boni; Tikuu (language of

Bajuni, dialect of Swahili)

Any relevant entries on the following subjects will be found subsumed into NORTH EAST AFRICA in volume 1: Unclassified p.524, Administration p.524, Agriculture p.524, Archaeology p.525, Art p.527, Chiefs p.529, Dancing p.530, Death p.530, Demography p.531, Economic; p.532, Education p.534, Folklore p.535, History p.536, Housing p.537, Islam p.537, Kinship p.538, Labour p.539, Land p.539, Law p.510, Marriage p.543, Medicine p.544, Missions, Christian p.515, Music p.546, Nutrition p.546, Religion p.547, Rock art p.547, Urban studies p.548, Women p.548

For other possible subjects, see the asterisked headings in Vol. 1, Section 1 


\section{CLASSIFIED CATALOGUE VOLUME 2}

\section{WEST AFRICA}

\section{Code-Number 4}

The arrangement of West Africa is as follows:

WEST AFRICA

Page

GAMBIA

SIERRA LEONE

62

GHANA

64

NIGERIA

(EX) AFRIQUE OCCIDENTALE

FRANCAISE

- MAURITANIE

- SENEGAL

- MALI

309

318

- NIGER

- GUINÉE

- COTE D'IVOIRE

- DAHOMEY (Benin Republic)

353

- HAUTE-VOLTA (Burkina Faso)

TOGO

371

CAMEROUN

LIBERIA

GUINÉ PORTUGUESA (Guiné-Bissau), CAPE VERDE, RIO DE ORO 


\section{WEST AFRICA}

\section{WEST AFRICA}

Unclassified

2 Administration (See also in vol.

2: The Gambia p.63, Sierra

Leone p.65, Ghana p.79, Nigeria p.126, Northern Nigeria p.189, Southern Nigeria p.217, (Ex) Afrique Occidentale Française p.249, Togo p.372, Cameroun p.386, Liberia p.422, Guiné Portuguesa p.432)

4 Agriculture (including Togo, Liberia, Guiné Portuguesa, Cape Verde, Rio de Oro. See also in vol. 2: The Gambia p.63, Sierra Leone p.66, Ghana p.84, Nigeria p.133, (ex) Afrique Occidentale Française p.255, Cameroun p.388)

6 Archaeology (including The Gambia, Sierra Leone, Togo, Cameroun, Liberia, Guiné Portuguesa, Cape Verde, Rio de Oro. See also in vol. 2: Ghana p.85, Nigeria p.136, (ex) Afrique Occidentale Française p.259)

7 Art (including The Gambia, Sierra Leone, Togo, Liberia, Guiné Portuguesa. Cape Verde, Rio de Oro. See also in vol. 2: Ghana p.86, Nigeria p.138, Afrique Occidentale Française p.263, Cameroun p.388)

9 Chiefs (including all countries and territories of West Africa)

13 Dancing (including all countries and territories of West Africa)

15 Death (including all countries and territories of West Africa)

18 Demography (including The Gambia, Sierra Leone, Togo, Liberia. See also in vol. 2: Ghana p.87, Nigeria p.145, Afrique Occidentale Française p.266, Cameroun p.389, Guiné Portuguesa / Cape Verde / Rio de Oro p.431 [out of order])
19 Economics (See also in vol. 2: The Gambia p.63, Sierra Leone p.66, Ghana p.89, Nigeria p.147, Afrique Occidentale Française p.268, Togo p.373, Cameroun p.390, Liberia p.423, Guiné Portuguesa p.432)

21 Education (including The Gambia, Guiné Portuguesa, Cape Verde, Rio de Oro. See also in vol. 2: Sierra Leone p.67, Ghana p.93, Nigeria p.156, Afrique Occidentale Française p.275, Togo p.373, Cameroun p.391, Liberia p.424)

Education, Higher (including all countries and territories of West Africa)

24 Ethnography (including Afrique Occidentale Française. See also in vol. 2: The Gambia p.63, Sierra Leone p.67, Ghana p.94, Nigeria p.160, Northern Nigeria p.191, Southern Nigeria p.220, Mauritania p.306, Senegal p.311, Mali p.319, Niger p.330, Guinée p.333, Côte d'Ivoire p.344, Dahomey p.354, Haute-Volta p.361, Togo p.373, Cameroun p.392, Liberia p.424, Guiné Portuguesa p.433, Cape. Verde p.437, Rio de Oro p.438)

- Unclassified ethnography

26 - Fulbe (Fula, Fulani, Peul) (see also in vol.2: Fulbe p.193, p.325, p.334)

27 - Mande (including Wangara) (see also in vol. 2: Malinke p.338)

Fishing (including The Gambia, Sierra Leone, Togo, Cameroun, Liberia, Guinté Portuguesa, Cape Verde, Rio de Oro; see also in vol. 2: Ghana p.11)8, Nigeria p.160, Afrique Occidentale Française p.277)

28 Folklore (including The Gambia, Liberia, Guiné Portuguesa, Cape Verde, Rio de Oro. See also in vol. 2: Sierra Leone p.72, Ghana 
p.108, Nigeria p.161, (ex)

Afrique Occidentale Française p.278, Togo p.380, Cameroun p.407)

29 History (See also in vol. 2: The Gambia p.63, Sierra Leone p.73, Ghana p.109, Nigeria p.163, (ex) Afrique Occidentale Française p.281, Togo p.380, Cameroun p.409, Liberia p.427, Guiné Portuguesa p.436)

32 Housing (including The Gambia, Sierra Leone, Ghana, Togo, Liberia, Guiné Portuguesa, Cape Verde, Rio de Oro. See also in vol. 2: Nigeria p.168, Afrique Occidentale Française p.287, Cameroun p.410)

33 Islam (including The Gambia, Sierra Leone, Togo, Cameroun, Liberia, Guiné Portuguesa, Cape Verde, Rio de Quo. See also in vol.2: Ghana p.111, Nigeria p.169, Afrique Occidentale Française p.288)

35 Kinship (including all countries and territories of West Africa)

37 Labour (including The Gambia, Sierra Leone, Togo, Liberia, Guiné Portuguesa, Cape Verde, Rio de Oro. See also in vol. 2: Ghana p.112, Nigeria p.170, Afrique Occidentale Française p.290, Cameroun p.410)

38 Land (including The Gambia, Sierra Leone, Togo, Liberia, Guiné Portuguesa, Cape Verde, Rio de Oro. See also in vol. 2: Ghana p.112, Nigeria p.172, (ex) Afrique Occidentale Française p.291)

39 Law (including The Gambia, Sierra Leone, Togo, Guiné Portuguesa, Cape Verde, Rio de Oro. See also in vol. 2: Ghana p.113, Nigeria p.174, (ex) Afrique Occidentale Française p.293, Cameroun p.410, Liberia p.428)
40 Linguistics (including The Gambia, Afrique Occidentale Française. See also in vol. 2: Sierra Leone p.73, Ghana p.115, Nigeria p.176, Northern Nigeria p.205, Southern Nigeria p.243, Mauritania p.308, Senegal p.317, Mali p.328, Niger p.332, Guinée p.341, Côte d'Ivoire p.352, Dahomey p.360, Haute-Volta p.369, Togo p.380, Cameroun p.411, Liberia p.428, Guiné Portuguesa p.416, Cape Verde Islands p.417)

- Unclassified linguistics

42 - (Ful, Fulani, Peul. See also in vol. 2: Fula p.206, p.341)

$43-$ Gur

44 - Kwa; Mande (Mandingo) (See also in vol. 2: Bambara p.328, Malinke p.342)

Marriage (including The Gambia, Sierra Leone, Togo, Liberia, Guiné Portuguesa, Cape Verde, Rio de Oro. See also in vol. 2: Ghana p.119, Nigeria p.177, Afrique Occidentale Française p.294, Cameroun p.417)

45 Medicine (including The Gambia, Sierra Leone, Togo, Liberia, Guiné Portuguesa, Cape Verde, Rio de Oro. See also in vol. 2: Ghana p.120, Nigeria p.178, Afrique Occidentale Française p.296, Cameroun p.418)

46 Missions (Christian) (including The Gambia, Togo, Liberia, Guiné Portuguesa, Cape Verde, Rio de Oro. See also in vol. 2: Ghana p.121, Nigeria p.179, Afrique Occidentale Française p.297)

47 Music (including The Gambia, Sierra Leone, Togo, Cameroun (see also p.419, 4th. item), Liberia, Guiné Portuguesa, Cape Verde, Rio de Oro. See also in vol.2: Ghana p.121, Nigeria 
p.181, Afrique Occidentale Française p.298)

48 Nutrition (including The Gambia, Sierra Leone, Ghana, Togo, Cameroun, Liberia, Guiné Portuguesa, Cape Verde, Rio de Oro. See also in vol. 2: Afrique Occidentale Française p.299)

49 Psychology (including all countries and territories of West Africa)

51 Race relations (including all countries and territories of West Africa)

Religion (including The Gambia, Guiné Portuguesa, Cape Verde, Rio de Oro. See also Sierra Leone p.77, Ghana p.122, Nigeria p.182, Afrique Occidentale Française p.300, Togo p.383, Cameroun p.419, Liberia p.431)

53 Rock art (including all countries and territories of West Africa)

54 Separatist movements (including all countries and territories of West Africa)

55 Social welfare (including all countries and territories of West Africa)

56 Urban studies (including all countries and territories of West Africa)

60 Women (including all countries and territories of West Africa)

For other possible subjects, see the asterisked headings in Vol. 1, Section 1

\section{GAMBIA}

\section{Code-number 41}

62 GAMBIA (For Senegambia, see under Senegal p.309 seq.)

Unclassified

63 Administration
Agriculture

Economics

Ethnography (See also in vol.2:

Malinke p.338)

- Unclassified ethnography History

Any relevant entries on the following subjects will be found subsumed into WEST AFRICA in volume 2: Unclassified p.1, Archaeology p.6, Art p.7, Chiefs p.9, Dancing p.13, Death p.15, Demography p.18, Economics p.19, Education p.21, Education, Higher p.21, Folklore p.28, Housing p.32, Islam p.33, Kinship p.35, Labour p.37, Land p.38, Law p.39, Linguistics p.40, Marriage p.44, Medicine p.45, Missions (Christian) p.46, Music p.47, Nutrition p.48, Psychology p.49, Race relations p.51, Religion p.51, Rock art p.53, Separatist movements p.54, Social welfare p.55, Urban studies p.56, Women p. 60

For other possible subjects, see the asterisked headings in Vol. 1, Section 1

\section{SIERRA LEONE}

\section{Code-number 42}

64 SIERRA LEONE

Unclassified

65 Administration

66 Agriculture

Economics

67 Education

Ethnography (See also in vol. 2:

Dyalonke p.334, Gola p.425,

Kru p.426, Susu p.340, Vai

p.427)

- Unclassified ethnography

68 - Bulom; Kono (see also in vol.

2: p.338); Koranko (Kuranko)

69 - Krio; Limba

70 - Mende (including Loko) 
71 - Temne (Timne) (including Banta)

72 Folklore

73 History

Linguistics

- Unclassified linguistics (see

also vol. 2: Susu p.343, Vai

p.430)

74 - Bulom (Sherbro) (including Krim); Koranko (Kuranko);

Krio; Limba

75 - Mende (including Loko)

76 - Temne (Timne)

Missions

77 Religion

Any relevant entries on the following subjects will be found subsumed into WEST AFRICA in volume 2:

Unclassified p.1, Administration p.2, Archaeology p.6, Art p.7, Chiefs p.9, Dancing p.13, Death p.15, Demography p.18, Education, Higher p.21, Fishing p.27, Housing p.32, Islam p.33, Kinship p.35, Labour p.37, Land p.38, Law p.39, Marriage p.44, Medicine p.45, Music p.47, Nutrition p.48, Psychology p.49, Race relations p.51, Rock art p.53, Separatist movements p.54, Social welfare p.55, Urban studies p. 56 , Women p. 60

For other possible subjects, see the asterisked headings in Vol. 1, Section 1

\section{GHANA (FORMERLY GOLD COAST)}

\section{Code-number 43}

$\begin{array}{ll}77 & \text { GHANA } \\ & \text { Unclassified } \\ 79 & \text { Administration } \\ 84 & \text { Agriculture } \\ 85 & \text { Archaeology } \\ 86 & \text { Art } \\ 87 & \text { Demography } \\ 89 & \text { Economics }\end{array}$

93 Education

94 Ethnography (see also in vol. 2: Ewe p.375, Kasena p.364, Lobi p.365, Wala p.369)

- Unclassified ethnography

96 - Adangme (including Krobo, Ningo, Shai)

97 - Akan (see also Akim, Ashanti, etc.)

98 - Akim (including Akim Abuakwa and Akim Kotoku); Ashanti

101 - Bedu; Brong (Bono) (including Nkwanta)

102 - Bulsa (Builsa); Dagari (Dagara, LoDagaba) (including Wiili, Wule or Ule); Dagomba (Dagbamba etc.)

103 - Denkyira; Fante (see also Twi)

104 - Fetu (Awutu, Effutu); Ga (including Teshi)

105 - Guang (Gonja) (including Krachi, Kyerepong, Larteh, Nkonya)

106 - Konkomba; Kusasi; Kwahu; Nafana (Mfantra); Nzima

107 - Osu; Sefwi; Talensi (Tallensi)

108 - Twi (including Akwapim, Akwamu)

Fishing

Folklore

109 History

111 Islam

112 Labour

Land

113 Law

115 Linguistics (See also in vol.2:

Anufo p.381, Ewe p.381, Grusi

p.370, Kulango p.352)

- Unclassified linguistics

115 - Adangme (including Krobo)

116 - Akan (see also Fante, Twi);

Dagari (Dagara); Dagomba

(Dagbane); Fante (see also

Twi)

117 - Ga; Guang (Gonja) (including Awutu, Lete, Nkunya)

118 - Gurenne; Isala (Sisala); 
Konkomba; Kusasi; Nafana; Nzima (Nzema) (including Ahanta); Twi (including Akwapem, Ashanti. See also Akan, Fante)

119 Marriage

120 Medicine

121 Missions Music

\section{Religion}

Any relevant entries on the following subjects will be found subsumed into WEST AFRICA in volume 2: Unclassified p.1, Chiefs p.9, Dancing p.113, Death p.15, Education, Higher p.21, Housing p.32, Kinship p.35, Nutrition p.48, Psychology p.49, Race relations p.51, Rock art p.53, Social welfare p.55, Urban studies p.56, Women p. 60

For other possible subjects, see the asterisked headings in Vol. 1, Section 1

\section{NIGERIA}

\section{Code-number 44}

124 NIGERIA (See also in vol. 2: Northern Nigeria p.187 seq., Southern Nigeria p.216 seq.) Unclassified

126 Administration (See also in vol. 2: Northern Nigeria p 189, Southern Nigeria p.217)

133 Agriculture (including Northern and Southern Nigeria)

136 Archaeology (including Northern and Southern Nigeria)

138 Art (including Northern and Southern Nigeria)

145 Demography (including Northern and Southern Nigeria)

147 Economics (including Northern and Southern Nigeria)
156 Education (including Northern and Southern Nigeria)

160 Ethnography (See also in vol. 2: Northern Nigeria p.191 seq., Southern Nigeria p.220 seq.) - Unclassified ethnography Fishing (including Northern and Southern Nigeria)

161 Folklore (including Northern and Southern Nigeria)

163 History (including Northern and Southern Nigeria)

168 Housing (including Northern and Southern Nigeria)

169 Islam (including Northern and Southern Nigeria)

170 Labour (including Northern and Southern Nigeria)

172 Land (including Northern and Southern Nigeria)

174 Law (including Northern and Southern Nigeria)

176 Linguistics (See also in vol. 2: Northern Nigeria p.205 seq., Southern Nigeria p.243 seq.) - Unclassified linguistics

177 Marriage (including Northern and Southern Nigeria)

178 Medicine (including Northern and Southern Nigeria)

179 Missions (including Northern and Southern Nigeria)

181 Music (including Northern and Southern Nigeria)

182 Nutrition (including Northern and Southern Nigeria) Religion (including Northern and Southern Nigeria)

Any relevant entries on the following subjects will be found subsumed into WEST AFRICA in volume 2: Unclassified p.1, Chiefs p.9, Dancing p.13, Death p.15, Education, Higher p.21, Kinship p.35, Psychology p.49, Race relations p.51, Rock art p.53, Separatist movements p.54, Social welfare p.55, Urban studies p.56, Women p.60 
For other possible subjects, see the asterisked headings in Vol. 1, Section 1

\section{NIGERIA, NORTHERN}

\section{Code-number 441}

\section{NIGERIA, NORTHERN} Unclassified

189 Administration

191 Ethnography (see also in vol. 2: Bariba p. 355, Dendi p.356, Dyerma p.331, Higi p.402, Songhai p.326

- Unclassified ethnography

192 - Angas; Bachama;

Basa-Komo; Bille; Birom;

Bolewa; Bura; Busa (Bussa) (including Kyenga);

Dakakari

193 - Fulbe (Fulani, Bororo) (See also in vol. 2: Fulbe p.26, p.331, p.401)

194 - Gade; Ginawuri; Gbari (Gwari); Hausa (including Zamfarawa)

199 - Idoma (including Afo); Igala (Igara)

200 - Igbira; Irigwe; Jaba; Jarawa; Jukun (Kororofawa); Kadara; Kagoro

201 - Kaleri (including Shalla); Kanuri (Bornu)

202 - Katab; Kofyar; Kulu; Kutep (Jompre); Lala; Mada (Nungu);

Margi (Marghi) (including Chibuk, Sukur)

203 - Nupe (including Kede); Rukuba

204 - Tangale; Tiv (Munshi)

205 Linguistics (see also in vol. 2: Bariba p.360, Dyerma p.332, Songhai p.329)

- Unclassified linguistics

206 - Angas (including Sura);

Bachama; Bade (group)

(including Bolanci (Hitkalanci), Karekare,

Ngizim); Birom; Busa (including Boko, Kyenga, Shanga); Etulo; Fula (Fulani, Fulfulde) (See also in vol. 2:

Fula p.42, p.415)

207 - Ganawuri (Aten); Gbari; Hausa

213 - Idoma (including Arago, Eloyi, Igede); Igala (Igara); Igbira; Jarawa; Jukun (Jukon) (including Jibu); Kambari (including Duka); Kanuri (Bornu) (including Badawi, Manga)

214 - Kofyar; Laamang; Maha; Margi (including Cibak or Chibuk); Nupe

215 - Reshe; Ron (including Sha); Sayanci; Tangale (including Kanakuru or Dera); Tera (including Ga'anda); TiV 216 - Tula; Yergum

For other possible subjects, see

NIGERIA, p. 124 seq.

\section{NIGERIA, SOUTHERN}

\section{Code-Number 442}

216 NIGERIA, SOUTHERN Unclassified

217 Administration

220 Ethnography (see also in vol. 2: Tiv p.204)

- Unclassified ethnography

221 - Agbor; Anaguta; Benin (including Bini [Edo]), Ishan, Weppa-Wano)

223 - Efik

224 - Ekoi; Ibibio (including Anang) (see also Efik)

225 - Igbo (Ibo) including Anam, Aro, Edda, Ika, Nri, Umundri)

230 - Ijo (Ijaw) (including Apoi) (see also: Kalabari p.231)

231 - Itsekiri (Jekri); Kalabari

232 - Mbembe; Sobo (including Isoko, Urhobo); Yakö

233 - Yoruba (including Egba, Ife, Ijebu [Yebu], Owo, Oyo. See 


\begin{tabular}{|c|c|}
\hline \\
\hline 43 & Linguistics (see also in vol. 2 : \\
\hline & Tiv p.215) \\
\hline & - Unclassified linguistics \\
\hline & $\begin{array}{l}\text { - Bini (Edo) (including Esako, } \\
\text { Kukuruku) }\end{array}$ \\
\hline 44 & - Ekoi (including Enyong, \\
\hline & Okonyong); Ibibio-Efik \\
\hline & (including Oron); Igbo (Ibo) \\
\hline & - Ijo (Ijaw) (including Nembe); \\
\hline & Izi; Mbembe; Nsibidi (secret \\
\hline & language and script); Obere \\
\hline & $\begin{array}{l}\text { Okaime ('spirit' language and } \\
\text { script); Ogoni; Sobo (Urhobo) }\end{array}$ \\
\hline & $\begin{array}{l}\text { Yoruba (including Yebu) (see } \\
\text { also in vol. 2: Nago p.361) }\end{array}$ \\
\hline
\end{tabular}

For other possible subjects, see NIGERIA, p.124 seq.

\section{(ex) AFRIQUE OCCIDENTALE FRANÇAISE (AOF)}

\section{Code-number 45}

249 (EX) AFRIQUE OCCIDENTALE FRANCCAISE (AOF) Unclassified

Subsumed here are all entries for Afrique Occidentale Française in general and for Mauritania, Senegal, Mali, Niger, Guinée, Côte d'Ivoire, Benin Republic and Burkina Faso on the following subjects:

$\begin{array}{ll}249 & \text { Administration } \\ 255 & \text { Agriculture } \\ 259 & \text { Archaeology } \\ 263 & \text { Art } \\ 266 & \text { Demography } \\ 268 & \text { Economics } \\ 275 & \text { Education } \\ 277 & \text { Fishing } \\ 278 & \text { Folklore } \\ 281 & \text { History } \\ 287 & \text { Housing }\end{array}$

288 Islam

290 Labour

291 Land

293 Law

294 Marriage

296 Medicine

297 Missions

298 Music

299 Nutrition

300 Religion

Any relevant entries on the following subjects will be found subsumed into WEST AFRICA in volume 2: Unclassified p.1, Chiefs p.9, Dancing p.13, Death p.15, Higher Education p.21, Ethnography p.24, Kinship p.35, Linguistics p.40, Psychology p.49, Race relations p.51, Rock art p.53, Separatist movements p.54, Social welfare p.55, Urban studies p.56, Women p. 60

For other possible subjects, see the asterisked headings in Vol. 1, Section 1

\section{MAURITANIE}

\section{Code-number 451}

305 MAURITANIE

Unclassified

306 Ethnology (see also in vol. 2:

Fulhe p.334, Kunta p.326,

Soninke (Sarakole) p.327,

Tuareg p.327, Wolof p.315)

- Unclassified ethnography

308 - Brakna: Hassanya; Imraguen;

Nemadi; Reguibat

Linguistics (see also in vol. 2:

Fula p.341, Soninke p.329,

Wolof p.317)

- Unclassified linguistics

309 - Hassanya; Zenaga

Any relevant entries on the following subjects will be found subsumed into AFRIQUE OCCIDENTALE FRANÇAISE in volume 2 : 
Unclassified p.249, Administration p.249, Agriculture p.255,

Archaeology p.259, Art p.263,

Demography p.266, Economics

p.268, Education p.275, Fishing

p.277, Folklore p.278, History

p.281, Housing p.287, Islam p.288,

Labour p.290, Land p.291, Law

p.293, Marriage p.294, Medicine

p.296, Missions p.297, Music

p.298, Nutrition p.299, Religion p.300

Any relevant entries on the following subjects will be found subsumed into WEST AFRICA in volume 2: Unclassified p.1, Chiefs p.9, Dancing p.13, Death p.15, Education, Higher p.21, Kinship p.35, Psychology p.49, Race relations p.51, Rock art p.53, Separatist movements p.54, Social welfare p.55, Urban Studies p.56, Women p.60

For other possible subjects, see the asterisked headings in Vol. 1. Section 1

\section{SÉNÉGAL}

\section{Code-number 452}

309 SÉNÉGAL

- Unclassified

311 Ethnography (see also in vol. 2:

Mauritanian ethnography p.306 seq., Balanta p.434, Bambara p.321, Fulbe p.334, Malinke

p.338, Soninke p.327)

- Unclassified ethnography

312 - Badyaranke Basari (see also in vol. 2: Konyagi p.338; Bedik;

Dyakanke; Dyola (Diola) (including Felupe)

314 - Lebu; Serer (including

Ndout, Non, Nyominka)

315 - Wolof (Ouolof)

317 Linguistics (see also in vol. 2: Bambara p.328, Fua p.341, Malinke p.342, Mandyak p.436, Soninke p.329)

- Unclassified linguistics

- Cangin; Dyola (Diola); Serer (including Kegem); Wolof (Ouolof)

Any relevant entries on the following subjects will be found subsumed into AFRIQUE OCCIDENTALE FRANÇAISE in volume 2:

Unclassified p.249, Administration p.249, Agriculture p.255, Archaeology p.259, Art p.263, Demography p.266, Economics p.268, Education p.275, Fishing p.277, Folklore p.278, History p.281, Housing p.287, Islam p.288, Labour p.290, Land p.291, Law p.293, Marriage p.294, Medicine p.296, Missions p.297, Music p.298, Nutrition p.299, Religion p. 300

Any relevant entries on the following subjects will be found subsumed into WEST AFRICA in volume 2 : Unclassified p.1, Chiefs p.9, Dancing p.13, Death p.15, Education, Higher p.21, Kinship p.35, Psychology p.49, Race relations p.51, Rock art p.53, Separatist movements p.54, Social welfare p.55, Urban Studies p.56, Women p.60

For other possible subjects, see the asterisked headings in Vol. 1. Section 1

\section{MALI (FORMERLY SOUDAN AND HAUT-SENEGAL- NIGER)}

\section{Code-number 453}

\section{MALI}

Unclassified

319 Ethnography see also in vol. 2: Dyula p.348, Kurumba p.364, Malinke p.338, Mossi p.366, Senufo p.350) 
Unclassified ethnography

321 - Bambara (Banmana) (including Kooroko)

322 - Bozo (Sorko, Sorkawa) (including Somono); Dogon (Habe)

325 - Dyawambe; Dyawara (Diawara); Fulbe (Peul, Tukulor) (see also in vol. 2: Fulbe p.26, p.193, p.331, p.334): Khasonke

326 - Kunta (nomads); Sonhai (Songai, Songhoi, Sonrai) (including Arma) (see also in vol. 2: Dyerma p.331, Dendi p.356)

327 - Soninke (Sanakole, Serahuli) (see also in vol 2: Marka p.366); Tuareg (including Bella and Western Iullemmeden (see also in vol. 1: Tuareg p.505; in vol. 2: Tuareg p.331)

328 Linguistics (see also in vol. 2: Fula p.42, Malinke p.338, Mossi p.370, Senufo p.352) Unclassified linguistics - Azer, Bambara (Banmana) (see also in vol. 2: Mande p.44); Bozo

329 - Dogon; Songhai (Songoi etc.) (see also in Vol. 2: Dyerma p.332, Soninke (Sarakole); Tuareg (see also in vol. 1: Tuareg p.510)

Any relevant entries on the following subjects will be found subsumed into AFRIQUE OCCIDENTALE FRANÇAISE in volume 2: Unclassified p.249, Administration p.249, Agriculture p.255, Archaeology p.259, Art p.263, Demography p.266, Economics p.268, Education p.275, Fishing p.277, Folklore p.278, History p.281, Housing p.287, Islam p.288, Labour p.290, Land p.291, Law p.293, Marriage p.294, Medicine p.296, Missions p.297, Music p.298, Nutrition p.299, Religion p. 300 Any relevant entries on the following subjects will be found subsumed into
WEST AFRICA in volume 2: Unclassified p.1, Chiefs p.9, Dancing p.13, Death p.15, Education, Higher p.21, Kinship p.35, Psychology p.49, Race relations p.51, Rock art p.53, Separatist movements p.54, Social welfare p.55, Urban Studies p.56, Women p.60

For other possible subjects, see the asterisked headings in Vol. 1, Section 1

\section{NIGER}

\section{Code-number 454}

\section{NIGER}

Unclassified

330 Ethnography (see also in vol. 2: Bozo (Sorkawa) p.322, Hausa p.194, Dendi p.356, Kanuri p.201, Teda p.494)

- Unclassified ethnography

331 - Azza; Buzu; Dyerma; Fulbe (including Wodaabe. See also in vol. 2: Fulbe p.26); Kurtey; Mawri; Sudie; Tuareg (including Eastern Iullemmeden) (see also in vol. 1: Tuareg p.505; in vol. 2: 327)

332 - Wogo

Linguistics (see also in vol. 2:

Fula p.206, Hausa p.207, Kanuri p.213, Teda p.497) - Dyerma (Zarma) (see also in vol. 2: Songhai p.329)

Any relevant entries on the following subjects will be found subsumed into AFRIQUE OCCIDENTALE FRANÇAISE in volume 2: Unclassified p.249, Administration p.249, Agriculture p.255, Archaeology p.259, Art p.263, Demography p.266, Economics p.268, Education p.275, Fishing p.277, Folklore p.278, History p.281, Housing p.287, Islam p.288, Labour p.290, Land p.291, Law p.293, Marriage p.294, Medicine p.296, Missions p.297, Music p.298, 
Nutrition p.299, Religion p.300

Any relevant entries on the following subjects will be found subsumed into WEST AFRICA in volume 2: Unclassified p.1, Chiefs p.9, Dancing p.13, Death p.15, Education, Higher p.21, Kinship p.35, Psychology p.49, Race relations p.51, Rock art p.53, Separatist movements p.54, Social welfare p.55, Urban Studies p.56, Women p.60

For other possible subjects, see the asterisked headings in Vol. 1, Section 1

\section{GUINÉE}

\section{Code-number 455}

\section{GUINÉE}

Unclassified

Ethnography (see also in vol. 2:

Koranko p.68, Dyola p.312,

Mano p.427)

- Unclassified ethnography

334 - Baga (Tyapi); Dyalonke (Yalunka); Fulbe (Fula, Peul) (including Fulakunda, Tukulor; see also in vol. 2: Fulbe p.26)

337 - Gerzé (Guerzé) (see also in vol. 2: Kpelle p.426); Kisi

338 - Kono (See also in vol. 2 :

Kono p.68); Konyagi (Coniagui) (including Basari)

(see also in vol. 2: Basari

p.312); Malinke (Mandingo,

Mandinka) (see also in vol. 2:

Mande p.27)

$340-$ Nalu; Susu (Soso)

341 - Tenda

Linguistics (see also in vol. 2:

Loma p.430)

- Baga (including Landuma);

Dyalonke (Yalunka); Fula

(Peul) (including Fulde, Pular,

Tukulor. See also in vol. 2:

Fula p.42)

342 - Gerzé (Guerzé) (see also in vol. 2: Kpelle p.429); Kisi:
Malinke (Mandingo,

Mandinka, Maninka) (see also in vol. 2: Mande p.44)

343 - Susu (Soso)

Any relevant entries on the following subjects will be found subsumed into AFRIQUE OCCIDENTALE FRANCCAISE in volume 2:

Unclassified p.249, Administration p.249, Agriculture p.255,

Archaeology p.259, Art p.263, Demography p.266, Economics

p.268, Education p.275, Fishing

p.277, Folklore p.278, History

p.281, Housing p.287, Islam p.288,

Labour p.290, Land p.291, Law

p.293, Marriage p.294, Medicine

p.296, Missions p.297, Music

p.298, Nutrition p.299, Religion

p. 300

Any relevant entries on the following subjects will be found subsumed into WEST AFRICA in volume 2: Unclassified p.1, Chiefs p.9, Dancing p.13, Death p.15, Education, Higher p.21, Kinship p.35, Psychology p.49, Race relations p.51, Rock art p.53, Separatist movements p.54, Social welfare p.55, Urban Studies p.56, Women p.60

For other possible subjects, see the asterisked headings in Vol. 1, Section 1

\section{CôTE D'IVOIRE}

\section{Code-number 456}

\section{COTE D'IVOIRE}

Unclassified

344 Ethnography (see also in vol. 2: Brong p.101, Dan p.425, Kono p.338, Kru p.426, Malinke

p.338, Nafana p.106, Nzima

p.106)

Unclassified ethnography

345 - Abure; Adyuku; Akye (Atye);

Aladyan (Aladian) 
346 - Anyi (Agni) (including

Abidji): Baule

347 - Bete (including Godye)

348 - Brong (Abron); Dida; Dyula

349 - Ebrie (including Aizi); Gagu;

Gere (see also in vol. 2: Kran

p.426); Guro

350 - Kulango; Tura; Ubi (Oubi);

Wobe; Senufo (Siena)

(including Dieli, Minianka,

Tagwana. Tiefo)

352 Linguistics (see also in vol. 2:

Dan p.429, Kru p.429,

Malinke p.342, Nafana p.118)

- Unclassified linguistics

- Abure; Adyukru; Akye

(Atye); Aladyan (Aladian);

Anyi (Agni); Avikam; Baule;

Bete; Ebrie; Kulango

(Kolango); Neyo; Senufo

(including Minianka,

Nyarafolo, Senadi, Tyembara,

Tagwana)

353 - Tura [Out of Order]

- Tenda (including

Badyaranke, Basari, Bedik)

Any relevant entries on the following subjects will be found subsumed into AFRIQUE OCCIDENTALE FRANCCAISE in volume 2 :

Unclassified p.249, Administration p.249, Agriculture p.255, Archaeology p.259, Art p.263, Demography p.266, Economics p.268, Education p.275, Fishing p.277, Folklore p.278, History p. 281 , Housing p.287, Islam p.288, Labour p.290, Land p.291, Law p.293, Marriage p.294, Medicine p.296, Missions p.297, Music p.298, Nutrition p.299, Religion p. 300

Any relevant entries on the following subjects will be found subsumed into WEST AFRICA in volume 2:

Unclassified p.1, Chiefs p.9, Dancing p.13, Death p.15, Education, Higher p.21, Kinship p.35, Psychology p.49, Race relations p.51, Rock art p.53,
Separatist movements p.54, Social welfare p.55, Urban Studies p.56, Women p.60

For other possible subjects, see the asterisked headings in Vol. 1, Section 1

\section{DAHOMEY (REPUBLIQUE DU BENIN)}

\section{Code-number 457}

\section{DAHOMEY}

Unclassified

354 Ethnography (see also in vol. 2:

Busa p.192, Ewe p.375, Gurma

p.363)

- Unclassified ethnography

355 - Adja; Aïzo (S. Alada); Bariba (Berba, Borgawa)

356 - Baseda (Winjiwinji);

Bazantche (Gbazantche);

Dendi, Fon (Fõ, Dahomean)

(including N. Alada)

357 - Gun

358 - Logba (Dompago); Mahi;

Nago (Yoruba peoples in

Dahomey) (including Dasa, Holli[dje], Ketu, Nyantruku, Shabe); Peda (Hweda)

359 - Pilapila (including Taneka);

Popo; Somba (group)

(including Besorube,

Betammaribe, Biyobe)

360 - Yoabu (Kwayaribe,

Tankamba)

Linguistics (see also in vol. 2:

Busa p.206, Ewe p.381, Fula

p.42, Gurma p.370

Adja; Bariba; Basila; Dendi;

Fon (Fõ, Dahomean); Gun

(Gũ)

- Unclassified linguistics

361 - Nago (Dahomey Yoruba)

Any relevant entries on the following subjects will be found subsumed into AFRIQUE OCCIDENTALE FRANCAISE in volume 2: 
Unclassified p.249, Administration

p.249, Agriculture p.255,

Archaeology p.259, Art p.263,

Demography p.266, Economics

p.268, Education p.275, Fishing

p.277, Folklore p.278, History

p.281, Housing p.287, Islam p.288,

Labour p.290, Land p.291, Law

p.293, Marriage p.294, Medicine

p.296, Missions p.297, Music p.298,

Nutrition p.299, Religion p. 300

Any relevant entries on the following

subjects will be found subsumed

into WEST AFRICA in volume 2:

Unclassified p.1, Chiefs p.9,

Dancing p.13, Death p.15,

Education, Higher p.21, Kinship

p.35, Psychology p.49, Race

relations p.51, Rock art p.53,

Separatist movements p.54, Social

welfare p.55, Urban Studies p.56,

Women p.60

For other possible subjects, see the

asterisked headings in Vol. 1 ,

Section 1

\section{HAUTE-VOLTA (BURKINA FASO)}

\section{Code-number 458}

361 HAUTE-VOLTA

Unclassified

Ethnography (see also in vol. 2:

Dagari p.102, Dyula p.348,

Fulbe p.325, Kusasi p.106,

Soninke p.327)

Unclassified ethnography

362 - Birifor; Bisa (Busanse) (see also in vol. 2: Busa p.192); Bobo (Bobo-Fing, Bobo-Ule) (including Bwa, Bdon, Bwaba. For Bobo-Dyula see in this volume: Dyula p.348)

363 - Doghosie; Dyan; Grusi

(Gurunsi); Gurma

(Gurmantche)

364 - Kasena; Kurumba

365 - Lobi (including Gan); Lyela (L'ela)
366 - Marka; Mossi (Moshi) (including Nyonyose)
368 - Pana
369 - Turuka (including Guin or Gwi); Tusia; Wala (including Natioro); Yarse
Linguistics (see also in vol. 2:
Isala p.188, Kusasi p.118)
- Unclassified linguistics
- Bisa (see also in vol. 2: Busa
p.206)
370 - Ble; Bo (Boka); Bobo
(including Bwamu); Dyan;
Gurma (Gurmantche); Grusi
(Gurunsi) (including Kasem or
Kasena, Nuna); Kurumba;
Lobi; Lyele (L'ele); Mossi
(Molé, Moré)
371 - Pana; Samo (San) (including Don)

Any relevant entries on the following subjects will be found subsumed into AFRIQUE OCCIDENTALE FRANÇAISE in volume 2:

Unclassified p.249, Administration p.249, Agriculture p.255, Archaeology p.259, Art p.263, Demography p.266, Economics p.268, Education p.275, Fishing p.277, Folklore p.278, History p.281, Housing p.287, Islam p.288, Labour p.290, Land p.291, Law p.293, Marriage p.294, Medicine p.296, Missions p.297, Music p.298, Nutrition p.299, Religion p.300

Any relevant entries on the following subjects will be found subsumed into WEST AFRICA in volume 2: Unclassified p.1, Chiefs p.9, Dancing p.13, Death p.15, Education, Higher p.21, Kinship p.35, Psychology p.49, Race relations p.51, Rock art p.53, Separatist movements p.54, Social welfare p.55, Urban Studies p.56, Women p.60

For other possible subjects, see the asterisked headings in Vol. 1, Section 1 
TOGO (INCLUDING EX BRITISH TRUST TERRITORY)

\section{Code-number 46}

\section{TOGO \\ Unclassified \\ 372 Administration \\ 373 Economics \\ Education}

Ethnography (see also in this volume: Adja p.355, Fon

p.356, Gurma p.363,

Konkomba p. 106, Kusasi

p.106, Nago p.358, Somba

p.359)

Unclassified ethnography

374 - Adele; Avatime; Bassari (Chambi)

375 - Bowiri, Ewe (including Anglo, Awuna, Glidyi, Mina) (see also in this volume: Popo p.359)

378 - Kabre (including Losso)

379 - Kebu (Akebu); Kposo (Akposso); Lefana (Lelemi); Moba; Naudeba; Tem (Temba, Kotokoli); Tyokossi

380 Folklore

\section{History}

Linguistics (see also in this volume: Adja p.360, Fon p.360, Gurma p.370, Konkomba p.188, Kusasi p.118)

- Unclassified linguistics

381 - Adele; Anufo (Tyokosi) (including Brissa); Avatime (including Nyangbo, Tafi);

Bassari (including Tobote); Bowiri; Ewe (Evhe) (including Ge, Mina, Popo)

383 - Kabre; Kasele (Tsamba); Kebu; Kposo; Lefana (Lelemi); Logba; Moba; Santrokofi; Tamari (Tamberna); Tem (including Lamba, Animere) Religion

Any relevant entries on the following subjects will be found subsumed into WEST AFRICA in volume 2: Unclassified p.1, Agriculture p.4, Archaeology p.6, Art p.7, Chiefs p.9, Dancing p.13, Death p.15, Demography p.18, Education, Higher p.21, Fishing p.27, Housing p.32, Islam p.33, Kinship p.35, Labour p.37, Land p.38, Law p.39, Marriage p.45, Medicine p.45, Missions (Christian) p.46, Music p.47, Nutrition p.48, Psychology p.49, Race relations p.51, Rock art p.53, Separatist movements p.54, Social welfare p.55, Urban Studies p.56, Women p. 60

For other possible subjects, see the asterisked headings in Vol. 1, Section 1

\section{CAMEROUN}

\section{Code-number 47}

385 CAMEROUN (General. See also in this volume: Ex-British Trust Territory p.420, exFrench Trust Territory p.421) Unclassified

386 Administration (including former Trust Territories)

388 Agriculture (including former Trust Territories Art (including former Trust Territories)

389 Demography (including former Trust Territories)

390 Economics (including former Trust Territories)

391 Education (including former Trust Territories)

392 Ethnography (see also in this volume: Babinga p.484, Baya p.499, Bayele p.652, Fang p.502, Bubi p.655, Kanuri p.201, Mada p.202, Margi p.202, Mundang p.493) - Unclassified ethnography

394 - Bafia (including Balom, Yambasa) 
395 - Bagam (Eghap); Bakoko;

Bali, Bamileke (including

Bangangte, Bangwa or Ngwe,

Banjun)

397 - Bamum

398 - Banen (including Ndiki);

Basa; Batanga (including

Banoho, Bapuku, Banaka);

Beti; Bulu (For Beti and Bulu see Beti)

399 - Duala

400 - Duru; Ekot Ngba; Esimbi;

Ewondo (Yaunde) (including

Bane, Evuzok)

401 - Fali; Fulbe (see also in this volume: Fulbe p.26, p.193)

402 - Gidar; Gisiga; Higi (Kapsiki);

Kaka; Kosi (Bakosi); Kotoko

(including Sao or So)

403 - Kpe (Bakweli, Bakweri,

Bakwiri); Kundu; Maka-Njem

(Dzem) (including Badjue,

Bikele, Ngumba)

404 - Mambila; Mandara; Masa

(including Banana-Kolon,

Marba, Tupuri); Matakam

(including Muktele, Mafa)

405 - Mbum; Mofu; Musgu;

Namchi (including Doayo);

Nyang (Banyang)

406 - Subu (Isubu, Bimbia); Tikar (group) (including Bafut, Eton, Kom or Nkom, Mbem, Nso,

Nsungli, Rom, Wiya)

407 - Tupuri (Tuburi); Vute

(Wute, Bute)

Folklore (including former

Trust Territories)

409 History (including former Trust Territories)

410 Housing (including former

Trust Territories)

Labour (including former Trust

Territories)

Law (including former Trust

Territories)

411 Linguistics (see also in this volume: Baya p.500, Fang

p.505, Kanuri p.213)

- Unclassified linguistics
412 - Bafia; Bagam (Eghap);

Bakoko; Bali; Bamileke

(including Bandem, Bangangte,

Bangwa or Ngwe)

413 - Bamum; Basa (including

Mbang); Bankon (Bo) (out of

order); Batanga (including

Banoho); Benue-Congo

(group); Bulu

414 - Daba; Duala; Ewondo

(Yaunde)

415 - Fali; Fuk; Fula (see also in

this volume: Fula p.42 and

p.206); Gisiga; Hide; Higi

(Kapsiki); Kosi (Nkosi);

Kotoko (including Logone, So)

416 - Kpe (Bakweli, Bakwiri);

Kundu; Lundu (Londo);

Mambai; Mambila; Mandara

(including Glavda, Paduko,

Yaghwatadaxa); Masa

(Masana) (including Musei);

Mbum (including Galke)

417 - Musgu (Musuk); Ngumba;

Nkom (Etung) (including

Bebejato, Gbedegi, Ngemba,

Nsaw, Tikar); Nyang

(including Mbe); Subu (Isubu);

Tunen (language of Banen);

Vute (Wute); Wori

Marriage (including former

Trust Territories)

418 Medicine (including former

Trust Territories)

Missions (including former

Trust Territories)

419 Music [4th. item (out of order).

For other possible entries see

vol. 2, p.47]

419 Religion (including former

Trust Territories)

Any relevant entries on the following subjects will be found subsumed into WEST AFRICA in volume 2:

Unclassified p.1, Archaeology p.6, Chiefs p.9, Dancing p.13, Death

p.15, Education, Higher p.21, Fishing p.27, Islam p.33, Kinship p.35, Music p.47, Nutrition p.48, 
Psychology p.49, Race relations p.51, Rock art p.53, Separatist movements p.54, Social welfare p.55, Urban studies p.56, Women p. 60

For other possible subjects, see the asterisked headings in Vol. 1, Section 1

\section{CAMEROONS}

\section{Code-number 471}

420 CAMEROONS (ex British Trust Territory)

Unclassified

\section{CAMEROONS}

Code-number 472

421 CAMEROONS (ex French Trust Territory)

Unclassified

\section{LIBERIA}

\section{Code-number 48}

\section{LIBERIA}

Unclassified

422 Administration

432 Economics

424 Education

Ethnography

- Unclassified

425 - Bandi (Gbande); Bassa (Mani); Belle; Dan; Gola

426 - Kpelle (see also in this volume: Gerze p.337); Kru (including Grebo, Jabo, Kran, Sabo)

427 - Loma (Toma); Mano; Vai (Gallinas)

428 Law

History

Linguistics

- Unclassified linguistics

429 - Bandi; Bassa; Dan (Gio);
Gola; Kpelle; Kru (including

Grebo, Gweabo, Pla)

430 - Loma (Toma); Mano; Manyua; Vai (including Dama)

431 Religion

Any relevant entries on the following subjects will be found subsumed into WEST AFRICA in volume 2: Unclassified p.1, Agriculture p.4, Archaeology p.6, Art p.7, Chiefs p.9, Dancing p.13, Death p.15, Demography p.18, Education, Higher p.21, Fishing p.27, Folklore p.28, housing p.32, Islam p.33, Kinship p.35, Labour p.37, land p.38, Marriage p.44, Medicine p.45, Missions (Christian) p.46, Music p.47, Nutrition p.48, Psychology p.49, Race relations p.51, Rock art p.53, Separatist movements p.54, Social welfare p.55, Urban studies p.56, Women p. 60

For other possible subjects, see the asterisked headings in Vol. 1, Section 1

\section{GUINÉ PORTUGUESA, CAPE VERDE ISLANDS, RIO DE ORO}

\section{Code-number 49}

[Only Demography is entered under this heading. For the other countries see in vol. 2: Guiné Portuguesa p.432, Cape Verde Islands p.437, Rio de Oro p.438]. Demography [Out of order: on p.431.]

\section{GUINÉ PORTUGUESA (GUINE-BISSAU)}

\section{Code-number 491}

(Includes works jointly on Guiné Portuguesa and Cape Verde 
Islands. For works on Cape Verde Islands alone see vol. 2: Cape Verde Islands, p.437 seq.)

432 GUINÉ PORTUGUESA

(Guiné-Bissau)

Unclassified

432 Administration

432 Economics

433 Ethnography (see also in this volume: Felupe p.312, Dyola p.312, Fulbe p.334, Malinke p.338, Nalu p.340)

434 - Balanta; Banyun (Banhun) (including Kassanga)

435 - Biafada; Bidyogo (Bijago); Brame; Kunante; Mandyak (Manjaco) (including Papel or Pepel)

436 History

436 Linguistics (see also in this volume: Dyola p.312, Fula p.341, Malinke (p.342)

- Unclassified linguistics

437 - Balanta; Biafada (Fada); Mandyak (including Brame, Mankanya, Papel or Pepel)

\section{CAPE VERDE ISLANDS}

\section{CAPE VERDE ISLANDS}

Code-number 492 (See also in vol. 2: Guiné Portuguesa p. 432 seq. for works jointly on Guiné Portuguesa and Cape Verde Islands)

CAPE VERDE ISLANDS

Unclassified

Ethnography

- Unclassified ethnography

Linguistics

- Unclassified linguistics

For other possible subjects, see the asterisked headings in Vol. 1, Section 1

\section{RIO DE ORO (SPANISH SAHARA)}

\section{Code-number 493}

438 RIO DE ORO (Spanish Sahara)

Unclassified

Ethnography (See also under Mauritania p.306)

- Unclassified ethnography

For other possible subjects, see the asterisked headings in Vol. 1, Section 1 


\section{WEST CENTRAL AFRICA: CODE-NUMBER 5}

The arrangement of West Central Africa is as follows:

WEST CENTRAL AFRICA

(EX) AFRIQUE

EQUATORIALE FRANÇAISE

TCHAD

REPUBLIQUE CENTRAFRICAINE

GABON

CONGO (ex-Congo-Brazzaville)

ZAIRE

ANGOLA

GUINEA ECUATORIAL

FERNANDO POO

SAO TOME AND PRINCIPE
Page

439

475

489

497

501

506

511

636

650

654

656

\section{WEST CENTRAL} AFRICA

\section{Code-number 5}

439 Unclassified

Agriculture (including Angola, Guinea Ecuatorial). See also in vol. 2: Afrique Équatoriale Française p.477, Zaïre p.520 Archaeology (including Angola, Guinea Ecuatorial). See also in vol. 2: Afrique Équatoriale Française p.478, Zaïre p.524

440 Art (including Angola, Guinea Ecuatorial). See also in vol. 2: Afrique Équatoriale Française p.479, Zaïre p.525

441 Chiefs (including all regions and countries of West Central Africa except São Tomé e Príncipe)

442 Dancing (including all regions and countries of West Central Africa except São Tomé e Príncipe)

443 Death (including all regions and countries of West Central Africa except São Tomé e Príncipe)

445 Demography (including
Angola, Guinea Ecuatorial). See also in vol. 2: Afrique Équatoriale Française p.480, Zaïre p.529

446 Economics (including Angola, Guinea Ecuatorial). See also in vol. 2: Afrique Équatoriale Française p.482, Zaïre p.533

447 Education (including all regions and countries of West Central Africa except São Tomé e Príncipe; Zaïre is included partly here and partly under ZAIRE itself, p.538).

448 Ethnography (See also in vol. 2: Afrique Equatoriale Française p.483, Zaïre p.541, Angola

p.638, Guinea Ecuatorial p.651)

448 - Unclassified ethnography

449 - Kongo (See also in vol. 2: p.507, p.611)

450 Fishing (including all regions and countries of West Central Africa except São Tomé e Príncipe)

451 Folklore (including Afrique Équatoriale Française, Angola, Guinea Ecuatorial). See also in vol. 2: Zaïre p.545

453 History (including Angola, 
Guinea Ecuatorial). See also in vol. 2: Afrique Équatoriale Française p.485, Zaïre p.550

455 Housing (including all regions and countries of West Central Africa except São Tomé e Príncipe)

456 Islam (including all regions and countries of West Central Africa except São Tomé e Príncipe)

Kinship (including all regions and countries of West Central Africa excepr São Tomé e Príncipe)

458 Labour (including Afrique Equatoriale Française, Angola, Guinea Ecuatorial. See also in vol. 2: Zaïre p.553)

459 Land (including Afrique Equatoriale Française, Angola, Guinea Ecuatorial. See also in vol. 2: Zaïre p.556)

Law (including Afrique Équatoriale Française, Angola, Guinea Ecuatorial. See also in vol. 2: Zaïre p.558)

460 Linguistics (See also in vol. 2: Afrique Équatoriale Française p.487, Zaiire p.564, Angola p.646, Guinea Ecuatorial p.653)

- Unclassified linguistics

- Kongo (See also in vol. 2: p.619)

Marriage (including Afrique Équatoriale Française, Angola, Guinea Ecuatorial. See also in vol. 2: Zaïre p.567)

461 Medicine (including Afrique Équatoriale Française, Angola, Guinea Ecuatorial. See also in vol. 2: Zaïre p.571)

463 Missions (including Guinea Ecuatorial. See also in vol. 2: Afrique Équatoriale Française p.487, Zaïre p.573, Angola p.648)
Music (including all regions and countries of West Central Africa except São Tomé e Príncipe)

465 Nutrition (including all regions and countries of West Central Africa except São Tomé e Príncipe)

467 Psychology (including all regions and countries of West Central Africa except São Tomé e Príncipe)

468 Race relations (including all regions and countries of West Central Africa except São Tomé e Príncipe) Religion (including Guinea Ecuatorial. See also in vol. 2: Afrique Équatoriale Française p.487, Zaire p.576, Angola p.649)

469 Rock art (including all regions and countries of West Central Africa except São Tomé e Príncipe)

470 Separatist movements (including all regions and countries of West Central Africa except São Tomé e Príncipe)

472 Social welfare (including Afrique Équatoriale Française, Angola, Guinea Ecuatorial. See also in vol. 2: Zaïre p.582) Urban studies (including all regions and countries of West Central Africa except São Tomé e Príncipe)

473 Women (including all regions and countries of West Central Africa except São Tomé e Príncipe)

For other possible subjects, see the asterisked headings in Vol. 1, Section 1 


\section{(EX) AFRIQUE EQUATORIALE FRANÇAISE (AEF)}

\section{Code-number 51}

475 Unclassified

476 Administration (including

Tchad, République

Centrafricaine, Gabon, Congo)

477 Agriculture (including Tchad,

République Centrafricaine,

Gabon, Congo)

478 Archaeology (including Tchad, République Centrafricaine, Gabon, Congo)

479 Art (including Tchad, République Centrafricaine, Gabon, Congo)

480 Demography (including Tchad, République Centrafricaine, Gabon, Congo)

482 Economics (including Tchad, République Centrafricaine, Gabon, Congo)

483 Ethnography (See also in vol. 2:

Tchad p.491, République

Centrafricaine p.498, Gabon

p.502, Congo p.506)

- Unclassified ethnography

484 - Babinga (pygmies) (including

Baka, Ngombe)

485 History (including Tchad, République Centrafricaine, Gabon, Congo)

487 Linguistics (See also in vol. 2:

Tchad p.495, République

Centrafricaine p.500, Gabon

p.505, Congo p.510,

Benue-Congo (group) p.413)

- Unclassified lir guistics

Missions (including Tchad,

République Centrafricaine,

Gabon, Congo)

Religion (including Tchad,

République Centrafricaine,

Gabon, Congo)

Any relevant entries on the following subjects will be found subsumed into WEST CENTRAL AFRICA

in volume 2: Chiefs p.441, Dancing

p.442, Death p.443, Education

p.447, Folklore 451, Housing

p.455, Islam p.456, Kinship p.456,

Labour p.458, Land p.459, Law

p.459, Marriage p.460, Medicine

p.461, Music p.463, Nutrition

p.465, Psychology p.467, Race

relations p.468, Rock art p.469,

Separatist movements p.470, Social welfare p.472, Urban studies p.472, Women p.473

For other possible subjects, see the asterisked headings in Vol. 1, Section 1

\section{TCHAD}

\section{Code-number 511}

489 Unclassified

491 Ethnography (See also in vol. 2:

Kotoko p.415, Masa p.416,

Tupuri p.407)

- Unclassified ethnography

492 - Bagirmi (including Bilala);

Bideyat (Baele); Buduma

(Yidena); Dai; Gula; Haddad;

Hadjerai (Saba)

493 - Kanembu; Mahamid;

Mundang; Sara

494 - Teda (including Daza,

Goran, Tebu, Tib(b)u,

Tub(b)u)

495 - Yalna; Zahawa

Linguistics

- Unclassified linguistics

496 - Bagirmi; Bideyat; Buduma;

Chadic (Chado-Hamitic);

Dangaleat; Gula; Jegu; Juman;

Kanembu

497 - Maba (Bura-Mabang); Mabar

(Mawer); Mimi; Nancere

(including Lele, Nantchoa);

Mubi [out of order]; Sara

(including Laka, Mbai); Tama

(including Sungor); Teda

(including Daza, Tubu) 
Any relevant entries on the following subjects will be found subsumed into WEST CENTRAL AFRICA in volume 2: Unclassified p.439, Chiefs p.441, Dancing p.442, Death p.443, Education p.447, Fishing p.450, Housing p.455, Islam p.456, Kinship p.456, Music p.463, Nutrition p.465, Psychology p.467, Race relations p.468, Rock art p.469, Separatist movements p.470, Urban studies p.472, Women p.473

Any relevant entries on the following subjects will be found subsumed into AFRIQUE EQUATORIALE FRANÇAISE in volume 2: Administration p.476, Agriculture p.477, Archaeology p.478, Art p.479, Demography p.480, Economics p.482, History p.485 For other possible subjects, see the asterisked heading sin Vol. 1, Section 1

\section{REPUBLIOUE CENTRAFRICAINE (ex Oubangui-Chari)}

\section{Code-Number 512}

497 Unclassified

498 Ethnography (See also in vol. 2: Babinga p.484; Bandia p.596; Ngbandi p.588; Mbuti p.600; Yakoma p.590; Sara p.493. For Zande, see vol. 1, p.586 and vol. 2 , p.602)

- Unclassified ethnography

- Banda (including Langba)

499 - Baya (Baja, Gbaya, Gbeya) (including Bofi. See also Manja); Gbanziri (Banziri); Manja (Mandj(i)a); Mbati (Isongo); Ngbaka (Lobaye)

500 - Nzakara

Linguistics (See also in vol. 2:

Ngbaka p.593; Ngbandi p.593;

Sara p.497. For Zande see vol.
1, p.593 and vol. 2, p.605)

- Unclassified linguistics

- Banda; Baya (Baja, Gbaya, Gbeya)

501 - Sango

Any relevant entries on the following subjects will be found subsumed into WEST CENTRAL AFRICA in volume 2: Unclassified p.439, Chiefs p.441, Dancing p.442, Death p.443, Education p.447, Fishing p.450, Nutrition p.465, Psychology p.467, Race relations p.468, Rock art p.469, Separatist movements p.470, Urban studies p.472, Women p.473

Any relevant entries on the following subjects will be found subsumed into AFRIQUE EQUATORIALE FRANCCAISE in volume 2:

Unclassified p.475, Administration p.476, Agriculture p.477,

Archaeology p.478, Art p.479, Demography p.480, Economics p.482, History p.485

For other possible subjects, see the asterisked headings in Vol. 1, Section 1

\section{GABON}

\section{Code-number: 513}

501 Unclassified

502 Ethnography (See also in vol. 2:

Babinga p.484; Bubi p.655;

Bula p.398; Maka-Njem p.403;

Teke p.508)

- Unclassified ethnography

- Duma; Fang (including

Ntum. See also in vol. 2: Fang p.652)

504 - Kota; Mbede (including Bamba); Myene; Nzabi, Punu (including Lumbu, Shira)

505 - Tsogo; Wandji

Linguistics (See also in vol. 2:

Bulu p.413; Teke p.510) 
- Unclassified linguistics

- Duma; Fang (See also

p.654); Kele; Mbede (including

Ndumu)

506 - Myene; Nzabi, Punu, Tsogo

Any relevant entries on the following subjects will be found subsumed into WEST CENTRAL AFRICA in volume 2: Unclassified p.439, Chiefs, p.441, Dancing p.442, Death p.443, Education p.447, Fishing p.450, Housing p.455, Islam p.456, Kinship p.456, Music p.463, Nutrition p.465, Psychology p.467, Race relations p.468, Rock art p.469, Separatist movements p.470, Urban studies p.472, Women p.473

Any relevant entries on the following subjects will be found subsumed into AFRIQUE EQUATORIALE FRANÇAISE in volume 2:

Unclassified p.475, Administration p.476, Agriculture p.477, Archaeology p.478, Art p.479, Demography p.480, Economics p.482, History p.485

For other possible subjects, see the asterisked headings in Vol. 1, Section 1

\section{CONGO (ex Congo-Brazzaville)}

\section{Code-number: 514}

506 Unclassified

Ethnography (See also in vol. 2:

Babinga p.484; Kota p.504;

Mbede p.504; Ngala p.586;

Punu p.504; Yombe p.617)

- Unclassified ethnography

507 - Babi; Bembe; Bomitaba;

Bomwali (Sangha-Sangha);

Bondjo; Bondongo; Bwende;

Dondo; Kamba; Kongo (see also in vol. 2: Kongo p.449, p.611)
508 - Lari; Mboshi (group) (including Kuba, Kuyu, Kwala, Makwa); Monjombo; Pomo;

Sundi; Teke (group) (including Boma, Kukuya, Lali, Ngangulu, Tyo)

509 - Vili (Fiote) (including Cabinda, Kakongo, Loango Ngoyo)

510 Linguistics (See also $\mathrm{n}$ vol. 2:

Mbede p.505; Punu p.506;

Ngala p.592; Yombe p.620)

- Unclassified linguistics

- Bembe; Lari (Laadi);

Mboshi; Teke (Tege) (group)

(including Fumu, Lali); Vili

(Fiote) (including Kakongo)

Any relevant entries on the following subjects will be found subsumed into WEST CENTRAL AFRICA in volume 2: Unclassified p.439; Chiefs p.441, Dancing p.442, Death p.443, Education p.447, Fishing p.450, Housing p.455, Islam p.456, Kinship p.456, Music p.463, Nutrition p.465, Psychology p.467, Race relations p.468, Rock art p.469, Separatist movements p.470, Urban studies p.472, Women p.473

Any relevant entries on the following subjects will be found subsumed into AFRIQUE EQUATORIALE FRANCAISE in volume 2:

Unclassified p.475, Administration p.476, Agriculture p.477, Archaeology p.478, Art p.479, Demography p.480, Economics p. 482 , History p. 485

For other possible subjects, see the asterisked heading sin Vol. 1, Section 1

\section{ZAIRE (ex- Congo-Kinshasa)}

Code-Number: 52

511 Unclassified 
512 Administration (including all regions of Zaïre)

520 Agriculture (including all regions of Zaïre)

524 Archaeology (including all regions of Zaïre)

525 Art (including all regions of Zaïre)

529 Demography (including all regions of Zaïre)

533 Economics (including all regions of Zaïre)

538 Education (including all regions of Zaïre; some education has been subsumed into West Central Africa, p.447)

541 Ethnography (See also in vol. 2 all regions of Zaïre: p.584, 594, $606,610,621,630$ )

- Unclassified ethnography

543 - Pygmy (including Baka, Efe).

See also in vol. 1: Pygmy p.177; see also in vol. 2: Mbuti p.600

- Twa (Batwa, Batswa)

(pygmies); see also in vol. 2:

Twa p.646

545 Folklore (including all regions of Zaïre)

550 History (including all regions of Zaïre)

553 Labour (including all regions of Zaire)

556 Land (including all regions of Zaïre)

558 Law (including all regions of Zaïre)

564 Linguistics (See also vol. 2 all regions of Zaïre p.590, p.603, p.608, p.618, p.626, p.635

- Unclassified linguistics

566 - Ngwana (Congo Swahili)

567 - Twa (Batwa, Batswa)

Marriage (including all regions of Zaïre)

571 Medicine (including all regions of Zaïre)

573 Missions (including all regions of Zaïre)

576 Religion (including all regions of Zaïre)
582 Social welfare (including all regions of Zaïre)

Any relevant entries on the following subjects will be found subsumed into WEST CENTRAL AFRICA in volume 2 : Unclassified p.439, Chiefs p.441, Dancing p.442, Death p.443, Education p.447, Fishing p.450, Housing p.455, Islam p.456, Kinship p.456, Music p.463, Nutrition p.465, Psychology p.467, Race relations p.468, Rock art p.469, Separatist movements p.470, Urban studies p.472, Women p.473

For other possible subjects, see the asterisked headings in Vol. 1, Section 1

\section{ZAIRE (cont.): Équateur, Province de 1'}

\section{Code-number: 521}

584 Unclassified

Ethnography (See also in vol. 2:

Gbanziri p.499, Ntomba

p.614)

- Unclassified ethnography

585 - Bangi (Mbangi, Bobangi);

Boloki; Budja; Doko; Furu,

Lalia; Mongo ('Lolo')

(Nkundo-Mongo). (See also

Nkundo)

586 - Mpama (Pama); Ngala

(including Banza, Leku)

587 - Ngbaka (Bwaka) (See also

Ngbaka, vol. 2, p.499)

588 - Ngbandi (inclúding Ngunda);

Ngombe (Gombe); Nkundo

(See also Mongo) (including

Edonda, Ngata)

589 - Nsamba; Nyi; Pagabete

590 - Poto (Foto); Sankani; Songo

(Nsongo?); Yakoma; Yela

Linguistics

- Unclassified linguistics

- Bangi (Bobangi) 
591 - Budja; Doko; Mongo ('Lolo') (Nkundo-Mongo) (See also Nkundo) (including Nkengo)

592 - Ngala (including Mabale, Mbanza, Mondunga (?))

593 - Ngbaka (Bwaka); Ngbandi; Ngombe; Nkundo (See also Mongo)

594 - Pagabete; Yela

Any relevant entries on the following subjects will be found subsumed into ZAIRE in volume 2 :

Unclassified p.511, Administration p.512, Agriculture p.520,

Archaeology p.524, Art p.525, Demography p.529, Economics p.533, Education p.538, Folklore p.545, History p.550, Labour p.553, land p.556, Law p.558, Marriage p.567, Medicine p.571, Missions p.573, Religion p.576, Social welfare p.582

Any relevant entries on the following subjects will be found subsumed into WEST CENTRAL AFRICA in volume 2: Unclassified p.439, Chief p.441, Dancing p.442, Death p.443, Education p.447, Fishing p.450, Housing p.455, Islam p.456, Kinship p.456, Music p.463, Nutrition p.465, Psychology p.467, Race relations p.468, Rock art p.469, Separatist movements p.470, Urban studies p.472, Women p.473

For other possible subjects, see the asterisked headings in Vol. 1, Section 1

\section{ZAIRE (cont.): (Haut-Zaire, ex-Prov. Orientale)}

\section{Code-number: 522}

594 Unclassified

Ethnography (See also in vol. 1: Bongo-Baka p.575, Kakwa p.578. See also in vol. 2 :
Nzakara p.500. See also in vol.

3: Lugbara p. 143)

- Unclassified ethnography

595 - Alur (including Angal, Jukot.

See also in vol. 3 Alur p.135);

Angba (including Beo,

Ngelima)

596 - Balese; Bali, Bandia

(Bandiya); Bangba (including

Angai); Barambo (Balambo)

597 - Bira (including Lengola); Bua (Ababua); Enya; Hima (Hema, Huma); Kango; Kare (Akare);

Kazibati

598 - Kebu (Ndo); Kele (Lokele)

(including Soko); Kumu;

Lendu (Bale); Logo-Avokaya

599 - Mamvu-Mangutu; Mangbetu (including Badjo (?), Matshaga

(?), Medje, Popoi, Rumbi);

Mbesa; Mbole (including

Foma)

600 - Mbuti (Bambuti) (pygmies)

(See also in vol. 2: Pygmy

p.543)

602 - Mituku; Ngwana (Arabisés);

Nyali (including Ndaka, bafueNuma); Poke (Topoke); Zande (Nyam-Nyam) (See also in vol. 1: Zande p.586; see also in vol. 2: Bandia p.596)

603 Linguistics (See also in vol. 3:

Lugbara p.157)

- Unclassified linguistics

604 - Alur (See also vol. 3, p. 155);

Angba (including Beo, Ngelima); Balese; Bira (including Lengola); Bua

(Ababua); Kele (Lokele) (including Olombo (Turumbu); So (Heso, Soko), Yalulema)

605 - Kumu; Lendu;

Logo-Avokaya; Mamvu (including Mabendi);

Mangbetu (including Makere, Medje); Mayogo; Mba; Mbesa; Mituku; Nyali (including bafueNuma); Zande (See also vol. 1, p.593) 
Any relevant entries on the following subjects will be found subsumed into ZAIRE in volume 2 :

unclassified p.511, Administration p.512, Agriculture p.520, Archaeology p.524, Art p.525, Demography p.529, Economics p.533, Education p.538, Folklore p.545, History p.550, Labour p.553, Land p.556, Law p.558, marriage p.567, Medicine p.571, Missions p.573, Religion p.576, Social welfare p.582

Any relevant entries on the following subjects will be found subsumed into WEST CENTRAL AFRICA in volume 2: Unclassified p.439, Chiefs 2:441, Dancing 2:442, Death 2:443, Education 2:447, Fishing 2:450, Housing 2:455, Islam 2:456, Kinship 2:456, Music 2:463, Nutrition 2:465, Psychology 2:467, Race relations $2: 468$, Rock art 2:469, Separatist movements 2:470, Urban studies $2: 472$, Women 2:473

For other possible subjects, see the asterisked headings in Vol. 1, Section 1

\section{ZAIRE (cont.): (Kivu, Province de)}

\section{Code-number: 523}

606 Unclassified

Ethnography (See also in vol. 3:

Amba p.136)

- Unclassified ethnography

- Bangubangu; Bembe; Buye;

Hunde; Lega (Rega)

607 - Nande, Ngengele (Gengele);

Nyanga; Shi (including

Nyabungu)

608 - Songola; Zimba (Binja)

Linguistics

- Unclassified linguistics

609 - Bangubangu; Bembe; Hunde; Lega (Rega); Nande; Nyanga; Ombo; Shi
Any relevant entries on the following subjects will be found subsumed into ZAIRE in volume 2 :

Unclassified p.511, Administration p.512, Agriculture p.520, Archaeology p.524, Art p.525, Demography p.529, Economics p.533, Education p.538, Folklore p. 545, History p.550, Labour p.553, Land p.556, law p.558, Marriage p.567, Medicine p.571, Missions p.573, Religion p.576, Social welfare p.582

Any relevant entries on the following subjects will be found subsumed into WEST CENTRAL AFRICA in volume 2: Unclassified p.439, Chief 2:441, Dancing 2:442, Death 2:443, Education 2:447, Fishing $2: 450$, Housing 2:455, Islam 2:456, Kinship 2:456, Music 2:463, Nutrition 2:465, Psychology 2:467, Race relations 2:468, Rock art 2:469, Separatist movements 2:470, Urban studies $2: 472$, Women 2:473

For other possible subjects, see the asterisked headings in Vol. 1, Section 1

\section{ZAIRE (cont.): (Bandundu et Zaire Inférieure) (ex Prov. de Léopoldville)}

\section{Code-number: 524}

609 Unclassified

610 Ethnography

- Unclassified ethnography

611 - Bomba (Buma); Djia (Badia, Edondja); Dzing; Holo; Humbu; Konda (Ekonda); Kongo (including Mbata, Tsamba. See also in vol. 2: Kongo p.449, Kongo p.507)

613 - Mbala; Mbun (Bunda, Mbunda)

614 - Mfinu (Nfunungu) (including Nfumu); Ntomba (including 
Bolia, Ntombe Njale, Sengele);

Oli; Pende

615 - Sakata (Lesa, Sa); Songo; Suku

616 - Yaka (Jaga, Maiaca) (including Lonso)

617 - Yansi; Yembe (Iyembe); Yombe

618 Linguistics

- Dzing

- Konda (Lokonda)

619 - Kongo (See also in vol. 2: Kongo p.460, Tuba p.620)

620 - Mfinu; Ntomba (including Bolia); Pende; Sakata; Tuba (Kituba); Yaka; Yansi (Bangi) (including Yei); Yombe

Any relevant entries on the following subjects will be found subsumed into ZAIRE in volume 2:

Unclassified p.511, Administration p.512, Agriculture p.520, Archaeology p.524, Art p.525, Demography p.529, Economics p.533, Education p.538, Folklore p.545, History p.550, labour p.553, Land p.556, Law p.558, Marriage p.567, Medicine p.571, Missions p.573, Religion p.576, Social welfare p.582

Any relevant entries on the following subjects will be found subsumed into WEST CENTRAL AFRICA in volume 2: Unclassified p.439, Chiefs 2:441, Dancing 2:442, Death 2:443, Education 2:447, Fishing 2:450, Housing 2:455, Islam 2:456, Kinship 2:456, Music 2:463, Nutrition 2:465, Pyschology 2:467, Race relations $2: 468$, Rock art $2: 469$, Separatist movements 2:470, Urban studies 2:472, Women 2:473

For other possible subjects, see the asterisked headings in Vol. 1, Section 1

\section{ZAIRE (cont.): (Kasai, Prov. du)}

\section{Code-number: 525}

621 Unclassified

Ethnography

- Unclassified ethnography

- Bushongo (Kuba) (including

Nyongo)

622 - Hamba; Kanyoka; Kela; Kete

623 - Lele (Bashi); Luba

(Luba-Lulua) (including

BakwaKalonji) (See also vol. 2:

Luba p.631)

624 - Lulua (Bena Lulua)

(including Bakwa Longo,

Bakwa Nyembi); Luntu (Bakwa

Luntu); Lwalwa; Mbagani

(Binji); Mbole (Bambuli)

625 - Milembwe; Nkutshu

(including Onga); Salampasu;

Songo (Basongo Meno)

(including Atshuru); Songye

(Songe) (including

BakwaLubo, Bena Bayashi,

Bena Ebombo, Bena Ngungi,

Sapo Sapo)

626 - Tetela

Linguistics

- Budya; Bushongo (Kuba)

(including Lele); Kanyoka

627 - Kete; Mbagani (Binji); Luba

(Luba Lulua) (See also in vol.

2: Luba p.635)

628 - Lulua (Bena Lulua)

629 - Milembwe; Nkutshu; Songye (Songe) (including Builande); Tetela

Any relevant entries on the following subjects will be found subsumed into ZAIRE in volume 2:

Unclassified p.511, Administration p.512, Agriculture p.520, Archaeology p.524, Art p.525, Demography p.529, Economics p.533, Education p.538, Folklore p.545, History p.550, Labour p.553, Land p.556, law p.558, 
Marriage p.567, Medicine p.571, Missions p.573, Religion p.576, Social welfare p.582

Any relevant entries on the following subjects will be found subsumed into WEST CENTRAL AFRICA in volume 2: Unclassified p.439, Chiefs 2:441, Dancing 2:442, Death 2:443, Education 2:447, Fishing 2:450, Housing 2:455, Islam 2:456, Kinship 2:456, Music 2:463, Nutrition 2:465, Psychology $2: 467$, Race relations $2: 468$, Rock art 2:469, Separatist movements $2: 470$, Urban studies $2: 472$, Women 2:473

For other possible subjects, see the asterisked headings in Vol. 1, Section 1

\section{ZAIRE (cont.): (Katanga, Prov. du)}

\section{Code-number: 526}

\section{Unclassified}

630 Ethnography (See also in vol. 2: Chokwe p.640. See also in vol. 3: Aushi p.291, Bemba p.291, Kaonde p.294, Lala p.294, Lamba p.295, Ndembu p.300)

- Unclassified ethnography

- Bena Muhona; Hemba (Luba-Hemba) (including Bena Kayumba)

631 - Holoholo (Horohoro); Kunda (Bakunda); Lomotwa; Luba

(See also vol. 2: Luba p.623) (including Samba)

633 - Lunda (including Temba) (See also vol. 3: Lunda p.633)

634 - Mitumba (Bena Mitumba); Ngoma (Bena Ngoma): Nkuvu (Bena Nkuvu); Sanga; Shila;

Tabwa

635 - Yeke; Zela

Linguistics

- Unclassified linguistics

- Bodo; Hemba

(Luba-Hemba); Holoholo;
Luba (including Samba) (See also vol. 2: Luba p.627)

636 - Lunda (See also vol. 3: Lunda p.307); Sanga; Tabwa

Any relevant entries on the following subjects will be found subsumed into ZAIRE in volume 2:

Unclassified p.511, Administration p.512, Agriculture p.520, Archaeology p.524, Art p.525, Demography p.529, Economics p.533, Education p.538, Folklore p.545, History p.550, Labour p.553, Land p.556, Law p.558, Marriage p.567, Medicine p.571, Missions p.573, Religion p.576, Social welfare p.582

Any relevant entries on the following subjects will be found subsumed into WEST CENTRAL AFRICA in volume 2: Unclassified p.439, Chiefs 2:441, Dancing 2:442, Death 2:443, Education 2:447, Fishing 2:450, Housing 2:455, Islam 2:456, Kinship 2:456, Music 2:463, Nutrition 2:465, Psychology 2:467, Race relations $2: 468$, Rock art 2:469, Separatist movements $2: 470$, Urban studies $2: 472$, Women 2:473

For other possible subjects, see the asterisked headings in Vol. 1, Section 1

\section{ANGOLA}

\section{Code-number 53}

636 Unclassified

637 Administration

638 Ethnography (See also in vol. 2: Kongo p.449, p.611, Songo p.615, Vili p.509, Yaka p.616, Yombe p.617. See also in vol. 3: Ambo p.505, Herero p.506, Ndembu p.300, Mbukushu p.518, Ndonga p.509. For Lunda see vol. 2 p.633, vol. 3 p.298) 
- Unclassified ethnography

640 - Bena Mai; Bungo; Chokwe (Cokwe, Kioko, Quioco)

642 - Diriku; Hanya; Hungu;

Konga (Kakonga); Kung (Bushman) (including Kazama

(?), Kwadi (?), Kwankala,

Sekwele. See also in vol. 3:

Kung p.507)

643 - Kwanyama (See also in vol. 3:

Kwanyama, p.508); Luchazi;

Luimbi; Lwena; Mbali;

Mbundu(ki) (including Mbaka

(Ambaquista), Mbangala,

Ngola)

644 - Mbundu(u) (including

Sambu, Wambu)

645 - Ngangela (Ganguela, Mbwela ?); Nyaneka (including Humbe (Kumbi), Mwila)

646 - Sama (Kisama); Solongo

(Sorongo); Sosso; Twa

(including Tyimba)

Linguistics

- Unclassified linguistics

647 - Chokwe; Diriku; Hungu;

Mbali; Mbunda; Mbundu(ki)

(including Mbangala. Mbui)

648 - Mbundu(u) (Bundo);

Ngangela; Nyaneka (including

Kumbi); Solongo (Sorongo)

Missions

\section{Religion}

Any relevant entries on the following subjects will be found subsumed into WEST CENTRAL AFRICA in volume 2: Unclassified p.439, Agriculture p.439, Archaeology p.439, Art p.440, Chiefs p.441, Dancing p.442, Death p.443, Demography p.445, Economics p.446, Education p.447, Fishing p.450, Folklore p.451, History p.453, Housing p.455, Islam p.456, Kinship p.456, Labour p.458, Land p.459, Law p.459, Marriage p.460, Medicine p.461, Music p.463, Nutrition p.465, Psychology p.467, Race relations p.468, Rock art p.469, Separatist movements p.470, Social welfare p.472, Urban studies p.472, Women p.473

For other possible subjects, see the asterisked headings in Vol. 1, Section 1

\section{GUINEA ECUATORIAL}

\section{Code-Number 54}

650 Unclassified

651 Administration (including

Fernando Poo and São Tomé and Príncipe)

Ethnography

- Unclassified ethnography

- Balengi

652 Bayele (Gyeli) (Pygmies);

Benga; Bujeba; Fang (see also in vol. 2: Fang p.502)

653 - Kombe

Linguistics

- Unclassified linguistics

- Basekwe

654 - Benga; Bujeba; Fang (Pamue) (see also in vol. 2: Fang p.505); Kombe

See also in vol. 2: Fernando Poo p. 654

Any relevant entries on the following subjects will be found subsumed into WEST CENTRAL AFRICA in volume 2: Unclassified p.439, Agriculture p.439, Archaeology p.439, Art p.440, Chiefs p.441, Dancing p.442, Death p.443, Demography p.445, Economics p.446, Education p.447, Fishing p.450, Folklore p.4.51, History p.453, Housing p.455, Islam p.456, Kinship p.456, Labour p.458, Land p.459, Law p.459, Marriage p.460, Medicine p.461, Missions p.463, Music p.463, Nutrition p.465, Psychology p.467, Race relations p.468, Religion p.4.68, Rock art p.469, Separatist movements p.470, 
Social welfare p.472, Urban studies p.472, Women p.473

For other possible subjects, see the asterisked headings in Vol. 1, Section 1

\section{FERNANDO POO, ANNOBON, CORISĊO}

\section{Code-Number 541}

\section{Unclassified}

655 Ethnography

- Unclassified ethnography

- Bubi

Linguistics

- Unclassified linguistics

- Bubi

See also in vol. 2: Administration p. 651

Any relevant entries on the following subjects will be found subsumed into WEST CENTRAL AFRICA in volume 2: Unclassified p.439, Chiefs p.441, Dancing p.442, Death p.443, Education p.447,
Fishing p.450, Housing p.455, Islam p.456, Kinship p.456, Music p.463 Nutrition p.465, Psychology p.467, Race relations p.468, Rock art p.460, Separatist movements p.470, Urban studies p.472, Women p.473

For other possible subjects, see the asterisked headings in Vol. 1, Section 1

\section{SAO TOME and PRINCIPE}

\section{Code-Number 542}

656 Ethnology

- Unclassified ethnography

- Angolares

Linguistics

- Unclassified linguistics

See also vol. 2, p.651, for

Administration subsumed into

Guinea Ecuatorial. For other possible subjects, see the asterisked headings in Vol. 1. Section 1 


\section{CLASSIFIED CATALOGUE VOLUME 3}

\section{EAST AFRICA}

\section{Code-Number 6}

The arrangement of East Africa is as follows:

\section{EAST AFRICA}

RWANDA AND BURUNDI

KENYA

UGANDA

TANZANIA

ZANZIBAR, PEMBA, MAFIA
Page

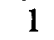

64

77

120

162

225

\section{EAST AFRICA}

\section{EAST AFRICA} Unclassified

2 Administration (including Zanzibar, Pemba, Mafia) (see also in this volume: Rwanda \& Burundi p.65, Kenya p.79, Uganda, p.121, Tanzania, p.164)

5 Agriculture (including Zanzibar, Pemba, Mafia) (see also in this volume: Rwanda \& Burundi p.68, Kenya p.84, Uganda, p.125, Tanzania, p.170)

6 Archaeology (including Rwanda, Burundi, Zanzibar-Pemba-Mafia) (see also in vol. 3: Kenya p.86, Uganda p.126, Tanzania p.173)

7 Art (including all countries of East Africa)

8 Chiefs (including all countries of East Africa)
10 Dancing (including all countries of East Africa)

11 Death (including all countries of East Africa)

12 Demography (including Rwanda, Burundi, Kenya, Zanzibar-Pemba-Mafia) (see also in this volume: Uganda p.128, Tanzania p.174)

14 Economics (including Zanzibar, Pemba, Mafia) (see also in this volume: Rwanda \& Burundi p.68, Kenya p.88, Uganda, p.129, Tanzania, p.175)

18 Education (including Rwanda, Burundi, Zanzibar-Pemba-Mafia) (see also in this volume: Kenya p.90, Uganda p.131, Tanzania p.179)

20 Education, Higher (including all countries of East Africa)

20 Ethnography (See also in this volume: Rwanda-Burundi p.69, 
Kenya p.91, Uganda p.133,

Tanzania p.181,

Zanzibar-Pemba-Mafia p.225)

Unclassified ethnography

22 - Indians; Nilo-Hamitic (including Kalenjin)

23 - Swahili (Shirazi) (including Unguja, Vumba) (see also in vol.

3: Amu p.92)

26 Fishing (including all countries of East Africa)

27 Folklore (including Zanzibar, Pemba, Mafia) (see also in this volume: Rwanda \& Burundi p.73, Kenya p.108, Uganda, p.149, Tanzania, p.206)

28 History (including Rwanda, Burundi, Zanzibar-Pemba-Mafia) (See also in this volume: Kenya p.109, Uganda p.150, Tanzania p.208)

31 Housing (including all countries of East Africa)

32 Islam (including all countries of East Africa)

34 Kinship (including all countries of East Africa)

36 Labour (including Rwanda, Burundi, Zanzibar-Pemba-Mafia) (See also in this volume: Kenya p.110, Uganda p.152, Tanzania p.210)

37 Land (including, Rwanda, Burundi, Zanzibar-Pemba-Mafia) (See also in this volume: Kenya p.110, Uganda p.153, Tanzania p.210)

38 Law (including Zanzibar, Pemba, Mafia) (see also in this volume: Rwanda \& Burundi p.73, Kenya p.112, Uganda p.154, Tanzania p.211)

39 Linguistics

39 - Unclassified linguistics
40 - Nilo-Hamitic (group) (including Kalenjin); Swahili (including Amu, Unguja, Vumba etc. See also in vol. 3: Ngazidja p.381, Nzwani p.381)

50 Marriage (including Kenya, Zanzibar-Pemba-Mafia. See also in this volume: Rwanda \& Burundi p.76, Uganda p.158, Tanzania p.220)

51 Medicine (including Rwanda-Burundi, Zanzibar-Pemba-Mafia. See also in this volume: Kenya p.117, Uganda p.159, Tanzania p.221)

52 Missions (including Zanzibar, Pemba, Mafia. See also in this volume: Rwanda \& Burundi p.77, Kenya p.118, Uganda p.160, Tanzania, p.222)

53 Music (including all countries of East Africa)

54 Nutrition (including all countries of East Africa)

57 Psychology (including all countries of East Africa)

Race relations (including all countries of East Africa)

58 Religion (including Rwanda, Burundi,

Zanzibar-Pemba-Mafia. See also in this volume: Kenya p.118, Uganda p.161, Tanzania p.222)

61 Rock art (including all countries of East Africa)

62 Separatist movements (including all countries of East Africa) Social Welfare (including all countries of East Africa) Urban Studies (including all countries of East Africa)

64 Women (including all countries of East Africa)

For other possible subjects, see the asterisked headings in Vol. 1, Section 1 


\section{RWANDA AND BURUNDI (ex-Ruanda-Urundi)}

Code-number 61

64 RWANDA AND BURUNDI (ex-Ruanda-Urundi) (For works on ex-German East Africa and ex Belgian Congo including

Ruanda-Urundi see in vol. 2 :

Zaïre p.511 séq., and in vol. 3:

Tanzania p. 162 seq.)

Unclassified

65 Administration

68 Agriculture

68 Economies

69 Ethnography (See also in vol. 2:

Twa p.543; in vol. 3: Kiga 141)

70 - Rundi

- Unclassified ethnography

71 - Rwanda (Ruanda, Nyarwanda)

72 - Tutsi (Tussi, Hima)

73 Folklore

Law

74 Linguistics (See also in vol. 2:

Ngwana (Congo Swahili)

p.566; Twa p.567)

- Unclassified linguistics

75 - Rundi; Rwanda (Ruanda, Nyarwanda)

76 Marriage

77 Missions

Any relevant entries on the following subjects will be found subsumed into EAST AFRICA in volume 3:

Unclassified p.1, Archaeology p.6, Art p.7, Chiefs p.8, Dancing p.10, Death p.11, Demography p.12, Education p.18, Education, Higher p.20, Fishing p.26, History p.28, Housing p.31, Islam p.32, Kinship p.34, Labour p.36, Land p.37, Medicine p.51, Music p.53, Nutrition p.54, Psychology p.57, Race relations p.57, Religion p.58, Rock art p.61, Separatist movements p.62, Social welfare p.62, Urban studies p.62, Women p.64
For other possible subjects, see the asterisked headings in Vol. 1. Section 1

\section{KENYA}

\section{Code-number 62}

77 KENYA

Unclassified

79 Administration

84 Agriculture

86 Archaeology

88 Economics

90 Education

91 Ethnography

- Unclassified ethnography

92 - Amu

93 - Dorobo (Okiek); Elmolo; Embu; Gumba (legendary); Gusii (Kisii) (including Logoli)

94 - Kamba (For works on Kamba and Kikuyu, see Kikuyu)

96 - Keyo (Elgeyo); Kikuyu (Gekoyo, Gikuyu)

99 - Kipsigis Lumbwa)

100 - Kony (Elgonyi); Luhya (Luyia, Bantu Kavirondo) (including Gwe, Hanga or Wanga, Samya, Tiriki)

101 - Luo (Nilotic Kavirondo) (see also in vol. 1: Lwo p.579)

102 - Masai (including Njemps) (See also in vol. 3: Masai p.194)

103 - Meru (including Mwimbi)

104 - Nandi; Nyika (Nika) (including Digo, Duruma, Giryama, Jomvu, Mnima, Ribe)

105 - Pokomo; Pokot (Pakot, Suk)

106 - Rendile; Samburu; Sanye (including Langulu); Sirikwa (legendary)

107 - Taita (Teita); Taveta; Tharaka (including Njuwe); Turkana

108 Folklore

109 History 


\section{Labour \\ Land \\ 112 Law \\ 113 Linguistics (See also in vol. 1: Galla p.623, Somali p.639; in vol.3: Swahili p.40) \\ - Unclassified linguistics \\ 114 -- Dorobo (Ndorobo, Okiek) (including Aramanik, Mosiro, Taturu); Gusii (including Logoli); Kamba; Kikuyu (Gekoyo, Gikuyu) \\ 115 - Luhya (Luyia) (including Nyole); Luo (Dho-Luo); Mogogodo; Nandi (including Kony) \\ 116 - Nyika (Nika) (including Digo, Giryama); Pokomo; Pokot (Suk) (including Endo); Rendile; Sanye; Taita (Teita) (including Dabida); Taveta; Thagicu \\ 117 - Tharaka; Turkana Medicine \\ 118 Missions Religion}

Any relevant entries on the following subjects will be found subsumed into EAST AFRICA in volume 3: Unclassified p.1, Art p.7, Chiefs p.8, Dancing p.10, Death p.11, Demography p.12, Education, Higher p.20, Fishing p.26, Housing p.31, Islam p.32, Kinship p.34, Marriage p.50, Music p.53, Nutrition p.54, Psychology p.57, Race relations p.57, Rock art p.61, Separatist movements p.62, Social welfare p.62, Urban studies p.62, Women p.64

For other possible subjects, see the asterisked headings in Vol. 1, Section 1

\section{UGANDA}

\section{Code-number 63}

120 UGANDA
Unclassified

121 Administration

125 Agriculture

126 Archaeology

128 Demography

129 Economics

131 Education

133 Ethnography (See also in vol. 1: Lwo p.579, Moru-Madi p.580, Nilotic p.581; in vol. 2 : Kebu p.598; in vol. 3: East African ethnography p.20, Rwanda (ethnic group) p.71, Luhya p.100, Pokot p.105)

- Unclassified ethnography

134 - Acoli (Acholi)

135 - Adhola (Padhola); Alur (See also in vol. 2: Alur p.595)

136 - Amba (and Bwisi); Ganda 140 - Gisu (Gesu, Geshu, Gishu) (including Bukusu); Hororo

(Mpororo); Karamojong (including Dodos, Ik, Jie, Moropom, Sorat [So], Teuso)

141 - Kenyi (Kene); Kiga (Chiga, Ciga)

142 - Koki; Konjo (Konzo);

Kumam; Labwor (and Nyakwai)

143 - Lango; Lugbara

144 - Nkole (Nyankole, Hima)

146 - Nyoro (Kitara)

147 - Sebei

148 - Sese (Sesse); Soga; Teso (Iteso)

149 - Toro; Vuma

Folklore

150 History

152 Labour

153 Land

154 Law

155 Linguistics (See also in vol. 1:

Lwo p.591, Moru-Madi p.591, Nilotic p.592; in vol. 3: East African linguistics p.39, Pokot p.116)

- Unclassified linguistics

155 - Acoli (Acholi, Acooli, Gang); Adhola (Jopadhola); Alur (see also in vol. 2: Alur p.604) 


\begin{aligned} & 156 - Ganda \\ & 157 - Gisu (Gishu, Masaba); \\ & Karamojong (Karimojong) \\ & (including Ik); Konjo (Konzo); \\ & Lango; Lugbara (Logbara, \\ & Madi); Nkore (Nkole, \\ & Nyankole, Nyankore) \\ & (including Kiga) \\ & 158 - Nyoro; Sabei; Soga; Syan \\ & (Orusyan); Teso (Ateso) \\ & 159 Marriage \\ & 160 Medicine \\ & 161 Missions \\ & \hline\end{aligned}

Any relevant entries on the following subjects will be found subsumed into EAST AFRICA in volume 3: Unclassified p.1, Art p.7, Chiefs p.8, Dancing p.10, Death p.11, Education, Higher p.20, Fishing p.26, Housing p.31, Islam p.32, Kinship p.34, Music p.53, Nutrition p.54, Psychology p.57, Race relations p.57, Rock art p.61, Separatist movements p.62, Social welfare p.62, Urban studies p.62, Women p.64

For other possible subjects, see the asterisked headings in Vol. 1. Section 1

\section{TANZANIA}

\section{Code-number 64}

162 TANZANIA (formerly Tanganyika and Zanzibar. For Zanzibar see vol. 3: p.225) Unclassified

164 Administration

170 Agriculture

173 Archaeology

174 Demography

175 Economics

179 Education

181 Ethnography (See also in vol. 2: Holoholo p.631; in vol. 3: East African ethnography p.20,
Dorobo p.93, Nyika p.104,

Luo p.101, Meru p.103,

Nyanja p.338, Makonde p.348,

Makua p.349, Yao p.353)

- Unclassified ethnography

183 - Asu (Wasu, Pare); Bena (See also Hehe)

184 - Bende; Bondei; Chaga (Chagga)

186 - Fipa

187 - Gogo; Ha

188 - Hadzapi (Hadza, Kangeju,

Kindiga, Tindega); Haya (Ziba) (including Hangiro, Mwani)

189 - Hehe (group) (including Kisi, Pangwa, Sangu) (See also Bena)

191 - Iraqw (including Goroa); Kaguru

193 - Kerewe including Kara); Kuria (including Simbiti); Kutu; Kwaya (Jita, Ruri) (including Ikizu, Sizaki); Langi (Rangi, Irangi); Luguru

194 - Masai (Kwavi) (including Arusha, Baraguyu, Kisonko.

See also in vol. 3: Masai p.102)

195 - Matengo

196 - Matumbi; Mbugu; Mbugwe; Mwanga (Namwanga) (and Iwa); Mwera; Ndamba; Ngasa; Ngindo; Ngoni (and

Ndendeuli) (See also in vol. 3: Ngoni p.337)

197 - Ngulu (Nguu, Nguru);

Nilyamba (Nyiramba,

Iramba, Isanzu); Nyakyusa

(Konde, Ngonde) (including Kinga)

199 - Nyamwezi (including Bungu, Kimbu, Konongo, Lwana, Sumbwa)

200 - Nyiha-Safwa (group)

201 - Pimbwe; Pogoro; Rimi (Nyaturu, Turu)

202 - Sandawe; Segeju; Shambala (Shambaa, Sambaa)

203 - Sonjo; Sukuma

205 - Tatoga (Datoga) (including 
Barabaig, Mangati); Vidunda;

Zanaki; Zaramo (including

Doe, Kwere, Shomvi)

206 - Zigua (Zegua, Zeguha,

Zigula); Zinza

206 Folklore

208 History

210 Labour

Land

211 Law

213 Linguistics (See also in vol. 3:

East African linguistics p.39,

Dorobo p.114, Nyika p.116,

Makua p.354, Yao p.357,

Makonde p.354)

- Unclassified linguistics

- Asu (Chasu, Pare)

214 - Bondei; Chaga (Chagga)

(including Machame, Moshi,

Siha); Fipa; Gogo (group)

(including Kaguru)

215 - Ha; Haya (Ziba); Hehe (group) (including Bena,

Pangwa); Iraqw (group)

(including Alagwa, Burungi or

Mbulunge, Goroa or Fiome)

216 - Kami; Kerewe; Kuria (Kulia);

Kwaya (Jita) (including

Shashi); Langi (Irangi); Luguru

(Ruguru); Masai (Maasai,

Kwafi. Loikop or Oigob);

Matengo

217 - Matumbi; Mbugu (including

Bwyo); Mbugwe (Buwe):

Mwera; Ngoni (Sutu);

Nilyamba (Ilamba, Iramba);

Nyakyusa (Konde, Ngonde)

(including Kinga); Nyamwezi

(including Kimbu, Lwana,

Sumbwa)

218 - Nyiha-Safwa (including

Lambya, Ndali); Pogoro; Rimi

(Limi, Nyaturu); Sandawe;

Shambala

219 - Sukuma; Tatoga (Taturu, Datoga); Zaramo (Dzalanio);

Zigula (Zigua)

220 - Zinza

Marriage

221 Medicine
$222 \underline{\text { Missions }}$

Religion

Any relevant entries on the following subjects will be found subsumed into EAST AFRICA in volume 3: Unclassified p.1, Art p.7, Chiefs p.8, Dancing p.10, Death p.11, Education, Higher p.20, Fishing p.26, Housing p.31, Islam p.32,

Kinship p.34, Music p.53,

Nutrition p.34, Psychology p.57, Race relations p.57, Rock art p.61, Separatist. movements p.62, Social welfare p.62, Urban studies p.62, Women p.64

For other possible subjects, see the asterisked headings in Vol. 1, Section 1

\section{ZANZIBAR, PEMBA, MAFIA}

\section{Code-number 65}

225 ZANZIBAR, PEMBA, MAFIA

(See also in vol. 3: East Africa

p. 1 seq., Tanzania p. 162 seq.)

- Unclassified

Ethnography (See also in vol. 3:

Swahili p.23)

- Unclassified ethnography

- Hadimu; Tumbatu

Linguistics (see also in vol. 3:

Swahili p.40)

- Unclassified linguistics

Any relevant entries on the following subjects will be found subsumed into EAST AFRICA in volume 3: Unclassified p.1, Administration p.2, Agriculture p.5, Archaeology p.6, Art p.7, Chiefs p.8, Dancing p.10, Death p.11, Demography p.12, Economics p.14, Education p.18, Education, Higher p.20, Fishing p.26, Folklore p.27, History p.28, Housing p.31, Islam p.32, Kinship p.34, Labour p.36, Land p.37, Law p.38, Marriage p.50, 
Medicine p.51, Missions p.52, Music p.53, Nutrition p.54, Psychology p.57, Race relations p.57, Religion p.58, Rock art p.61, Separatist movements p.62, Social welfare p.62, Urban studies p.62, Women p.64

For other possible subjects, see the asterisked headings in Vol. 1, Section 1 


\section{SOUTH-EAST CENTRAL AFRICA}

\section{Code-number 7}

The arrangement of the South-East Central Africa is as follows:

$\begin{array}{lc} & \text { Page } \\ \text { SOUTH-EAST CENTRAL AFRICA } & 226 \\ \text { EX RHODESIA, North and South } & 281 \\ \text { ZAMBIA } & 281 \\ \text { RHODESIA (Zimbabwe) } & 309 \\ \text { MALAWI (ex-Nyasaland } & 332 \\ \text { MOÇAMBQUE } & 342 \\ \text { MADAGASCAR } & 357 \\ \text { COMORO ISLANDS } & 381\end{array}$

\section{SOUTH-EAST CENTRAL AFRICA}

\section{SOUTH-EAST CENTRAL}

AFRICA

Unclassified

Administration (See also in this volume: Zambia p.282,

Rhodesia (Zimbabwe) p.310, Malawi p.333, Moçambique p.343, Madagascar p.359)

228 Agriculture (including Malawi, Moçambique. See also in this volume: Zambia p.284, Rhodesia (Zimbabwe) p.311, Madagascar p.361)

229 Archaeology (including Malawi, Moçambique and Madagascar) (See also Zambia p.286, Rhodesia (Zimbabwe) p.312)

231 Art (including all countries of South-East Central Africa)

232 Chiefs (including all countries of South-East Central Africa)

233 Dancing (including all countries of South-East Central Africa)

234 Death (including all countries of South-East Central Africa)

236 Demography (including Zambia, Rhodesia (Zimbabwe), Malawi and Moçambique) (See also in this volume: Madagascar p.362)
238 Economics (including Malawi and Moçambique) (See also in this volume: Zambia p.287, Rhodesia (Zimbabwe) p.315, Madagascar p.363)

240 Education (including all countries of South-East Central Africa)

244 Ethnography (See also all countries of South-East Central Africa)

- Unclassified ethnography

- Maravi (Malawi)

Fishing (including all countries of South-East Central Africa)

245 Folklore (including all countries of South-East Central Africa)

248 History (including Zambia, Rhodesia (Zimbabwe), Malawi and Moçambique) (see also in this volume: Madagascar p.374)

251 Housing (including all countries of South-East Central Africa)

252 Islam (including all countries of South-East Central Africa) Kinship (including all countries of South-East Central Africa)

254 Labour (including all countries of South-East Central Africa)

257 Land (including all countries of South-East Central Africa) 
259 Law (including all countries of South-East Central Africa)

262 Linguistics (see also in this volume: all countries of South-East Central Africa) - Unclassified linguistics Marriage (including all countries of South-East Central Africa)

264 Medicine (including all countries of South-East Central Africa)

266 Missions (including Zambia, Rhodesia (Zimbabwe), Malawi and Moçambique) (see also in this volume: Madagascar p.379)

269 Music (including all countries of South-East Central Africa)

271 Nutrition (including all countries of South-East Central Africa)

272 Psychology (including all countries of South-East Central Africa)

273 Race relations (including all countries of South-East Central Africa)

275 Religion (including Malawi and Moçambique) (see also in this volume: Zambia p.308, Rhodesia (Zimbabwe) p.331, Madagscar p.379)

276 Rock art (including Zambia, Rhodesia (Zimbabwe), Malawi and Moçambique)

277 Separatist movements (including all countries of South-East Central Africa)

278 Social welfare (including all countries of South-East Central Africa)

279 Urban studies (including all countries of South-East Central Africa)

280 Women (including all countries of South-East Central Africa)

For other possible subjects, see the asterisked headings in Vol. 1, Section 1

\section{ex RHODESIA, North and South}

Code-number 71

\section{ZAMBIA}

\section{Code-number 711}

\section{ZAMBIA \\ Unclassified \\ 282 Administration \\ 284 Agriculture \\ 286 Archaeology \\ 287 Economics}

290 Ethnography (See also in vol. 3:

Fipa p.186, Mwanga p.196, Cewa p.336; Kwe p.518)

- Unclassified ethnography

- Ambo

291 - Aushi (Ushi); Bemba (including Chishinga, Ngoma)

293 - Bisa (For Bisa and Bemba, see Bemba)

294 - Ila (Shukulumbwe); Kaonde; Lala

295 - Lamba; Lozi (Rotse, Luyana, Kololo) (including Subiya)

298 - Lunda (of Kazembe) (See also in vol. 2: Lunda p.633; in vol. 3: Luvale p.299)

299 - Luvale (Lovale, Lunda-Lovele, Wiko) (See also in vol. 2: Lwena p.643)

300 - Mambwe: Ndembu

(Ndembo); Ngoni (See also in vol.3: Ngoni p.337)

301 - Nkoya (Mbwela); Nsenga; Sala

302 - Senga; Soli; Tonga (General and Plateau Tonga) (including Toka)

303 - Tonga (Valley Tonga or We) (See also in vol. 3: Tonga p.329)

304 - Twa; Unga

Linguistics

- Unclassified linguistics 


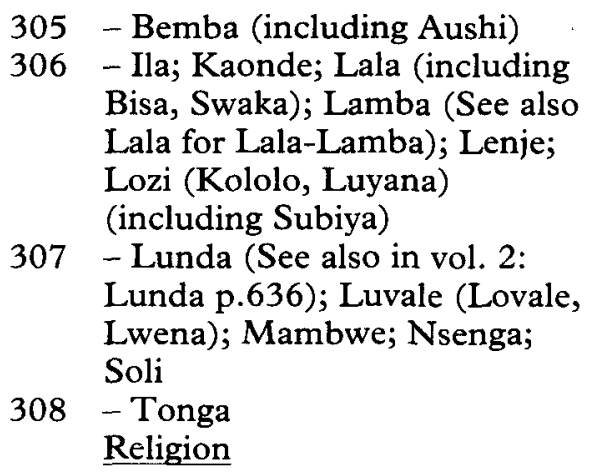

Any relevant entries on the following subjects will be found subsumed into SOUTH-EAST CENTRAL AFRICA in volume 3: Unclassified p.226, Art p.231, Chiefs p.232, Dancing p.233, Death p.234, Demography p.236, Education p.240, Fishing p.244, Folklore p.2.45, History p.248, Housing p.251, Islam p.252, Kinship p.252, Labour p.254, Land p.257, Law p.259, Marriage p.262, Medicine p.264, Missions p.266, Music p.269, Nutrition p.271, Psychology p.272, Race relations p.273, Rock art p.276, Separatist movements p.277, Social welfare p.278, Urban studies p. 279 , Women p. 280

For other possible subjects, see the asterisked headings in Vol. 1, Section 1

\section{RHODESIA (Zimbabwe) 712}

309 RHODESIA (Zimbabwe) Unclassified

310 Administration

311 Agriculture

312 Archaeology

315 Economics

316 Ethnography (See also in this volume: Barwe p.346, Lemba p.477, Ndau p.349, Thonga p. 351 , Venda p.48!, Zulu p.485)
- Unclassified ethnography

318 - Budya (Buja); Duma; Hera (and Njanja); Jawunda;

Kalanga (Kalana)

319 - Karanga (See also Duma, Mari, Rozwi)

320 - Korekore; Manyika (including Ungwe [Hungwe])

321 - Mari; Mbire; Nanzwa; Ndebele (Matabele)

324 - Rembutu (Lembutu); Rozwi [both ancient and existing peoples of that name; including Nohwe. See also in this volume: Karanga p.319)]

325 - Shankwe: Shawasha; Shona (including Dumbuseya, Goba)

329 - Tawara; Tonga (Toka, Tonka) (see also in this volume: Tonga p.302); Zezuru Linguistics

- Unclassified linguistics

- Karanga

330 - Manyika; Ndebele; Shona (including Lilima. See also Zezuru and other dialects)

331 - Zezuru (Swina)

\section{Religion}

Any relevant entries on the following subjects will be found subsumed into SOUTH-EAST CENTRAL AFRICA in volume 3: Unclassified p.226, Art p.231, Chiefs p.232, Dancing p.233, Death p.234, Demography p.236, Education p.240, Fishing p.244, Folklore p.245, History p.248, Housing p.251, Islam p.252, Kinship p.252, Labour p.254, Land p.257, Law p.259, Marriage p.262, Medicine p.264, Missions p.266, Music p.269, Nutrition p.271, Psychology p.272, Race relations p.273, Rock art p.276, Separatist movements p.277, Social welfare p.278, Urban studies p.279, Women p.280

For other possible subjects, see asterisked headings in Vol. 1, Section 1 


\section{MALAWI (ex Nyasaland)}

\section{Code-number 72}

332 MALAWI (ex Nyasaland)

Unclassified

333 Administration

335 Ethnography (See also in this volume: Maravi p.244,

Nyakusa p.197)

- Unclassified ethnography

336 - Cewa (Chewa)

337 - Kunda; Ngoni (See also in this volume: Ngoni p.196 and p.300; Nguni p.418)

338 - Nguru (See also in this volume: Lomwe p.348, Makua p.349); Nyanja (including Ma(n)ganja, Nyasa)

339 - Tonga; Tumbuka (including Henga, Kamanga); Yao (See also in this volume: Yao p.353)

340 Linguistics

- Unclassified linguistics

- Cewa (Chewa); Ngoni;

Nyanja (including Mang'anja, Nyasa. See also Cewa)

342 - Tumbuka (including Henga, Kamanga, Tonga); Yao (See also in this volume: Yao p.357)

Any relevant entries on the following subjects will be found subsumed into SOUTH-EAST CENTRAL AFRICA in volume 3: Unclassified p.226, Agriculture p.228, Archaeology p.229, Art p.231, Chiefs p.232, Dancing p.233, Death p.234, Demography p.236, Economics p.235, Education p.240, Fishing p.2.44, Folklore p.245, History p.248, Housing p.251, Islam p.252, Kinship p.252, Labour p.254, Land p.257, Law p.259, Marriage p.262, Medicine p.264, Missions p.266, Music p.269, Nutrition p.271, Psychology p.272, Race relations p.273, Religion p.278, Rock art p.276, Separatist movements p.277, Social welfare p.278, Urban studies p.279, Women p.280

For other possible subjects, see the asterisked headings in Vol. 1, Section 1

\section{MOÇAMBIQUE}

\section{Code-number 73}

\section{MOÇAMBIQUE}

Unclassified

343 Administration

344 Ethnography (See also in this volume: Swahili p.23, Maravi

p.244, Nsenga p.301, Manyika

p.320, Tawara p.329, Cewa

p.336, Ngoni p.331, Nyanja

p.338, Swazi p.526)

- Unclassified ethnography

346 - Barwe; Chopi (Lenge)

347 - Chwabo (Acwabo)

348 - Lomwe; Makonde

349 - Makua (including Chirima); Ndau

350 - Nyungwe; Ronga

351 - Sena; Teve (including Gorongozi); Thonga (Tonga, Tsonga, Shangaan)

353 - Tswa; Yao (See also in this volume: Yao p.339); Zimba (See also in this volume:

Vazimba p.374)

354 Linguistics (See also in this volume: Nsenga p.307;

Manyika p.330)

- Unclassified linguistics

- Chopi (Lenge); Chwabo;

Lomwe (Lolo); Makonde;

Makua

355 - Maviha; Ndau; Nyungwe

(Tete); Ronga

356 - Sena; Thonga (Tsonga)

(including Gwamba)

357 - Tonga (GiTonga); Tswa; Yao (See also in this volume: Yao p.342) 
Any relevant entries on the following subjects will be found subsumed into SOUTH-EAST CENTRAL AFRICA in volume 3: Unclassified p.226, Agriculture p.228, Archaeology p.229, Art p.231, Chiefs p.232, Dancing p.233, Death p.234, Demography p.236, Economics p.238, Education p.240, Fishing p.244, Folklore p.245, History p.248, Housing p.251, Islam p.252, Kinship p.252, Labour p.254, Land p.257, Law p.259, Marriage p.262, Medicine p.264, Missions p.266, Music p.269, Nutrition p.271, Psychology p.272, Race relations p.273, Religion p.275, Rock art p.276, Separatist movements p.277, Social welfare p.278, Urban studies p.279, Women p. 280

For other possible subjects, see the asterisked headings in Vol. 1, Section 1

\section{MADAGASCAR}

\section{Code-number 74}

\section{MADAGASCAR}

Unclassified

359 Administration (including Comoro Islands)

361 Agriculture (including Comoro Islands)

362 Demography (including Comoro Islands)

363 Economics (including Comoro Islands)

365 Ethnography (See also Comoro Islands)

- Unclassified ethnography

368 - Antambahoaka

(Tambahooka); Antandroy

(Tandroy); Antankarana

(Tankarana); Antanosy (Tanosi)

369 - Antefasi (Antaifasy, Tefasy); Antemoro (Antaimoro,
Temoro); Antesaka (Antaisaka, Tesaka); Bara

370 - Betsileo: Betsimisaraka; Bezanozano

371 - Mahafaly; Marofotsy; Merina (Andriana, Hova)

372 - Sakalava

373 - Sihanaka; Tanala (including Zafimaniry)

374 - Tsimihety (For works on Tsimihety and Sakalava, see Sakalava); Vazimba; Vezo History (including Comoro Islands)

376 Linguistics - Unclassified linguistics

379 Missions (including Comoro Islands)

Religion (including Comoro Islands)

Any relevant entries on the following subjects will be found subsumed into SOUTH-EAST CENTRAL AFRICA in volume 3: Unclassified p.226, Archaeology p.229, Art p.231, Chiefs p.232, Dancing p.233, Death p.234, Education p.240, Fishing p.244, Folklore p.245, Housing p.251, Islam p.252, Kinship p.252, Labour p.254, Land p.257, Law p.259, Marriage p.262, Medicine p.264, Music p.269, Nutrition p.271, Psychology p.272, Race relations p.273, Separatist movements p.271, Social welfare p.278, Urban studies p.279, Women p. 280

For other possible subjects, see the asterisked headings in Vol. 1, Section 1

\section{COMORO ISLANDS}

Code-number 741

COMORO ISLANDS

Unclassified

Ethnology 
- Unclassified ethnography

Linguistics

- Unclassified linguistics

- Ngazidja; Nzwani

Any relevant entries on the following subjects will be found subsumed into SOUTH-EAST CENTRAL AFRICA in volume 3: Unclassified p.226, Archaeology p.229, Art p.231, Chiefs p.232, Dancing p.233, Death p.234, Education p.240, Fishing p.244, Folklore p.245, Housing p.251, Islam p.252, Kinship p.252, Labour p.254, Land p.257, Law p.259, Marriage p.262,
Medicine p.264, Music p.269, Nutrition p.271, Psychology p.272, Race relations p.273, Separatist movements p.271, Social welfare p.278, Urban studies p.279, Women p. 280

See also in vol. 3 the following subjects incorporated into MADAGASCAR: Administration p.359, Agriculture p.361, Demography p.362, Economics p.363, History p.374, Missions p. 379, Religion p.379

For other possible subjects, see the asterisked headings in Vol. I, Section 1 


\title{
382 SOUTHERN AFRICA
}

\author{
Code-number 8
}

The arrangement of Southern Africa is as follows:

SOUTHERN AFRICA

UNION OF SOUTH AFRICA

SOUTH WEST AFRICA

(Namibia)

BOTSWANA

LESOTHO

SWAZILAND

\section{Page \\ 382 \\ 476 \\ 501 \\ 515 \\ 522 \\ 526}

\section{SOUTHERN AFRICA}

\section{SOUTHERN AFRICA}

Unclassified

384 Administration (See also in this volume: Transvaal p.476, Natal p.484, Cape Province p.492, S.W. Africa (Namibia) p.502, Ex-British Protectorates (Botswana, Lesotho, Swaziland) p.512)

388 Agriculture (including all countries of Southern Africa)

390 Archaeology (including all countries of Southern Africa)

394 Art (including all countries of Southern Africa)

Chiefs (including all countries of Southern Africa)

396 Dancing (including all countries of Southern Africa) Death (including all countries of Southern Africa) Demography (including all countries of Southern Africa)

398 Economics (including all countries of Southern Africa)

404 Education (including all countries of Southern Africa)

409 Education, Higher (including all countries of Southern Africa)

410 Ethnography (See also in vol. 3: Transvaal p.477, Natal, p.484, Cape Province p.493,
South West Africa (Namibia) p.504, Botswana p.516, Lesotho p.522, Swaziland p.526))

- Unclassified ethnography

413 - Bushman (See also in this volume: Bushman p.516, Khoisan p.418)

415 - Coloured

417 - Indian

418 - Khoisan (Bushman and Hottentot) (See also in this volume: Bushman p.413, Hottentot p.495); Nguni

419 - Sotho-Tswana (See also in this volume: Sotho p.479 and p.523; Tswana p.519); Tlokwa Folklore (including all countries of Southern Africa)

422 History (inc. all countries of Southern Africa)

426 Housing (including all countries of Southern Africa)

427 Kinship (including all countries of Southern Africa)

428 Labour (including all countries of Southern Africa)

432 Land (including all countries of Southern Africa

434 Law (including all countries of Southern Africa)

437 Linguistics (See also in this volume: Transvaal p.482, Natal p.489, Cape Province p.498, South West Africa (Namibia) 
p.509, Botswana p.521,

Lesotho p.524, Swaziland

p.327)

- Unclassified linguistics

439 - Afrikaans; Bushman

(including $\neq$ Auni,

$\neq$ Khomani, Sarwa)

440 - Fanagalo (Basic Bantu,

Kitchen or Mine Kafir);

Khoisan (Bushman and

Hottentot) (See also in this

volume: Bushman p.439,

Hottentot p.498); Nguni

441 - Sotho-Tswana

Marriage (including all countries of Southern Africa)

443 Medicine (including all countries of Southern Africa)

446 Missions (including all countries of Southern Africa)

450 Music (including all countries of Southern Africa)

451 Nutrition (including all countries of Southern Africa)

452 Psychology (including all countries of Southern Africa)

453 Race relations (including all countries of Southern Africa)

465 Religion (including all countries of Southern Africa)

468 Rock art (including all countries of Southern Africa)

471 Separatist movements (including all countries of Southern Africa)

472 Social welfare (including all countries of Southern Africa)

474 Urban studies (including all countries of Southern Africa)

475 Women (including all countries of Southern Africa)

For other possible subjects, see the asterisked headings in Vol. 1, Section 1

\section{UNION OF SOUTH AFRICA}

\author{
Code-number 81
}

\section{UNION OF SOUTH AFRICA}

See: Transvaal p.476, Orange Free State p.484, Natal p.484, Cape Province p.492

Any relevant entries on the following subjects will be found subsumed into SOUTHERN AFRICA in volume 3: Unclassified p.382, Administration p.384, Agriculture p.388, Archaeology p.390, Art p.394, Chiefs p.394, Dancing p.396, Death p.396, Demography p.396, Economics p.398, Education p.404, Education, Higher p.409, Folklore p.419, History p.422, Housing p.426, Kinship p.427, Labour p.428, Land p.432, Law p.434, Marriage p.441, Medicine p.443, Missions p.446, Music p.450, Nutrition p.451, Psychology p.452, Race relations p.453, Religion p.465, Rock art p.468, Separatist movements p.471, Social welfare p.472, Urban studies p.474, Women p. 475

For other possible subjects, see the asterisked headings in Vol. 1, Section 1

\section{TRANSVAAL}

\section{Code-number 811}

476 TRANSVAAL

Unclassified

Administration

477 Ethnography (see also in this volume: Fokeng p.522, Kgatla

p.517, Kwena p.518, Malete

p.518, Rembutu p.324, Rolong

p.519, Swazi p.526, Thonga

p.351, p.481, Tlokwa p.419,

Tswana p.519)

- Unclassified ethnography 
- Birwa; Hurutse; Lemba (Lepa, Remba)

478 - Lobedu (Lovedu)

479 - Ndzundza Pedi; Sotho (North) (including Khaha, Letsoalo, Malaboch

(Gananwa), Masemola, Ntshabeleng, Phalaborwa, Roka, Tau) (see also in this volume: Pedi p.479)

481 - Thonga (See also in this volume: Thonga p.351); Venda

482 Linguistics

- Lobedu (Lovedu); Ndebele (including Ndzundza) (see also in this volume: Ndebele p.330); Sotho (North) (Pedi)

483 - Thonga (See also in this volume: Thonga p.356); Venda

Any relevant entries on the following subjects will be found subsumed into SOUTHERN AFRICA in volume 3: Unclassified p.382, Agriculture p.388, Archaeology p.390, Art p.394, Chiefs p.394, Dancing p 396, Death p.396, Demography p.396, Economics p.398, Education p.404, Education, Higher p.409, Folklore p.419, History p.422, Housing p.426, Kinship p.427, Labour p.428, Land p.432, Law p.434, Marriage p.441, Medicine p.443, Missions p.446, Music p.450, Nutrition p.451, Psychology p.452, Race relations p.453, Religion p.465, Rock art p.468, Separatist movements p.471, Social welfare p.472, Urban studies p.474, Women p.475

For other possible subjects, see the asterisked headings in Vol. 1, Section 1

\section{ORANGE FREE STATE}

\section{Code-number 812}

ORANGE FREE STATE Unclassified
Any relevant entries on the following subjects will be found subsumed into SOUTHERN AFRICA in volume 3: Unclassified p.382, Agriculture p.388, Archaeology p.390, Art p.394, Chiefs p.394, Dancing p.396, Death p.396, Demography p.396, Economics p.398, Education p.404, Education, Higher p.409, Folklore p.419, History p.422, Housing p.426, Kinship p.427, Labour p.428, Land p.432, Law p.434, Marriage p.44!, Medicine p.443, Missions p.446, Music p.450, Nutrition p.451, Psychology p.452, Race relations p.453, Religion p.465, Rock art p.468, Separatist movements p.471, Social welfare p.472, Urban studies p.414, Women p.475

For other possible subjects, see the asterisked headings in Vol. 1, Section 1

\section{NATAL}

\section{Code-number 813}

484 NATAL

Unclassified

Administration

Ethnography (see in this volume: Baca p.494, Indian p.417, Sotho (South) p.523, Swazi p.526)

- Unclassified

485 - Kanya (Makanya); Ngwane; Zulu

489 Linguistics - Lala; Zulu .

Any relevant entries on the following subjects will be found subsumed into SOUTHERN AFRICA in volume 3: Unclassified p.382, Agriculture p.388, Archaeology p.390, Art p.394, Chiefs p.394, Dancing p.396, Death p.396, Demography p.396, Economics p.398, Education p.404, Education, 
Higher p.409, Folklore p.419, History p.422, Housing p.426, Kinship p.427, Labour p.428, Land p.432, Law p.434, Marriage p.441, Medicine p.443, Missions p.446, Music p.450, Nutrition p.451, Psychology p.452, Race relations p.453, Religion p.465, Rock art p.468, Separatist movements p.471, Social welfare p.472, Urban studies p.474, Women p.475

For other possible subjects, see the asterisked headings in Vol. 1, Section 1

\section{CAPE PROVINCE}

\section{Code-number 814}

492 CAPE PROVINCE

Unclassified

Administration

493 Ethnography (see in this volume: Bushman p.413, p.516, Coloured p.415, Nama p.508)

- Unclassified ethnography

494 - Baca (amaBaca, Bhaca);

Bomvana; Fingo (abaMbo, Mfengu); Hlubi

495 - Hottentot (including Grikwa. See also in this volume:

Khoisan p.418, Korana p.496, Nama p.508)

496 - Korana; Mpondo; Thembu; Tlhaping

497 - Xhosa

498 Linguistics

- Hottentot (including Grikwa.

See also in this volume:

Khoisan p.440, Korana (on this page), Nama p.511); Korana 499 - Mpondo; Xhosa (Kafir)

Any relevant entries on the following subjects will be found subsumed into SOUTHERN AFRICA in volume 3: Unclassified p.382, Agriculture p.388, Archaeology p.390, Art p.394, Chiefs p.394, Dancing p.396, Death p.396,
Demography p.396, Economics p.398, Education p.404, Education, Higher p.409, Folklore p.419, History p.422, Housing p.426, Kinship p.427, Labour p.428, Land p.432, Law p.434, Marriage p.441, Medicine p.443, Missions p.446, Music p.450, Nutrition p.451, Psychology p.452, Race relations p.453, Religion p.465, Rock art p.468, Separatist movements p.471, Social welfare p.472, Urban studies p.474, Women p.475

For other possible subjects, see the asterisked headings in Vol. 1.

Section 1

\section{SOUTH WEST AFRICA (Namibia)}

\section{Code-number 82}

501 SOUTH WEST AFRICA

(Namibia)

Unclassified

502 Administration

504 Ethnography (see in this volume: Bushman p.413, p.516, Coloured p.415, Hottentot p.495, Korana p.496, Mbukushu p.518) - Unclassified ethnography

505 - Ambo (Ovambo, group) (See also in this volume: Kwanyama p.508, Ndonga p.509)

506 - Bergdama; Herero (including Tjimba)

507 - Kung (!Kung) (including Heikum, !Kau, !Ko, !Xũ. See also in volume 2: Kung p.642)

508 - Kwanyama (Kwanyama Ambo) (See also in volume 2: Kwanyama p.643); Nama (Khoi-Khoi, Namakwa, Naman, Nama-Hottentot) (including Gai-Khan (Rooi Natie), Topnaar. See also in this volume: Khoisan p.418, Hottentot p.495) 
509 - Ndonga

Linguistics- Unclassified linguistics

- Ambo (Ovambo, group) (See also in this volume: Kwanyama p.510, Ndonga p.512)

510 - Herero; Kung (!Kung);

Kwangali; Kwanyama

511 - Nama (Khoi-Khoi, Namakwa, Nama-Hottentot). (See also in this volume: Khoisan p.440, Hottentot p.498)

512 - Ndonga including Kwambi)

Any relevant entries on the following subjects will be found subsumed into SOUTHERN AFRICA in volume 3: Unclassified p.382, Agriculture p.388, Archaeology p.390, Art p.394, Chiefs p.394, Dancing p,396, Death p.396, Demography p.396, Economics p.398, Education p.404, Education, Higher p.409, Folklore p.419, History p.422, Housing p.426, Kinship p.427, Labour p.428, Land p.432, Law p.434, Marriage p.441, Medicine p.443, Missions p.446, Music p.450, Nutrition p.451, Psychology p.452, Race relations p.453, Religion p.465, Rock art p.468, Separatist movements p.471, Social welfare p.472, Urban studies p.474, Women p.475

For other possible subjects, see the asterisked headings in Vol. 1, Section 1

\section{EX-BRITISH PROTECTORATES}

\section{Code-number 83}

512 Ex-British protectorates (see also in vol. 3: Botswana p.515 seq., Lesotho p.522 seq., Swaziland p.526 seq.) Unclassified Administration (including Botswana, Lesotho, Swaziland)

\section{BOTSWANA (ex-Bechuanaland)}

\section{Code-number 831}

\section{BOTSWANA}

(ex-Bechuanaland)

Unclassified

516 Ethnography (see also in this volume: Birwa p.477, Bushman p.413, Herero p.506, Hurutshe p.477, Kalanga (Kalana) p.318, Kung p.507, Tlokwa p.419) - Unclassified ethnography - Bushman (including Auen, $\neq$ Auni, G/wi, $\neq$ Khomani, Kxoe, Naron. See also in this volume: Bushman p.413, Kwe p.518, Sarwa p.519)

517 - Kgalagadi; Kgatla (Kxatla)

518 - Kwe (Hukwe Bushmen);

Kwena (Koena); Malete; Mbukushu; Ngwaketse

519 - Ngwato: Rolong (including Kaa); Sarwa (Masarwa, Tati Bushmen); Tawana; Tswana (Bechuana, Chwana) (see also in this volume: Sotho-Tswana p.419)

521 Linguistics (see in this volume: Bushman p.439, Kwangali p.510)

- Unclassified linguistics

- Tswana (Sechuana) (see also in this volume Sotho-Tswana p.441

Any relevant entries on the following subjects will be found subsumed into SOUTHERN AFRICA in volume 3: Unclassified p.382, Agriculture p.388, Archaeology p.390, Art p.394, Chiefs p.394, Dancing p.396, Death p.396, Demography p.396, Economics P.398, Education p.404, Education, Higher p.409, Folklore p.419, History p 422, Housing p.426, Kinship p.427, Labour p.478, Land p.472, Law p.434, 
Marriage p.441, Medicine p.443, Missions p.446, Music p.450, Nutrition p.451, Psychology p.452, Race relations p.453, Religion p.465, Rock art p.468, Separatist movements p.471, Social welfare p.472, Urban studies p.474, Women p.475

For other possible subjects, see the asterisked headings in Vol. 1, Section 1

\section{LESOTHO (ex Basutoland) (832)}

522 LESOTHO (ex Basutoland) Unclassified

Ethnography (see in this volume: Hurutse p.477, Kung p.507, Kwena p.518, Tlokwa p.419)

- Unclassified ethnography

- Fokeng; Khwakhwa

523 - Sotho (Southern) (Basuto)

524 Linguistics

Unclassified linguistics

- Phuthi

525 - Sotho (Southern) (Sesuto)

Any relevant entries on the following subjects will be found subsumed into SOUTHERN AFRICA in volume 3: Unclassified p.382, Agriculture p.388, Archaeology p.390, Art p.394, Chiefs p.394, Dancing p.396, Death p.396, Demography p.396, Economics p.398, Education p.404, Education, Higher p.409, Folklore p.419, History p.422, Housing p.426, Kinship p.427, Labour p.428, Land p.432, Law p.434, Marriage p.441, Medicine p.443, Missions p.446, Music p.450, Nutrition p.451, Psychology p.452, Race relations p.453, Religion p.465, Rock art p.468, Separatist movements p.471, Social welfare p.472, Urban studies p.474, Women p.475
For other possible subjects. see the asterisked headings in Vol. 1, Section 1

\section{SWAZILAND}

\section{Code-number 833}

526 SWAZILAND

Unclassified

Ethnography

- Swazi

527 Linguistics

- Swazi

Any relevant entries on the following subjects will be found subsumed into SOUTHERN AFRICA in volume 3: Unclassified p.382, Agriculture p.388, Archaeology p.390, Art p.394, Chiefs p.394, Dancing p.396, Death p.396, Demography p.396, Economics p.398, Education p.404, Education, Higher p.409, Folklore p.419, History p.422, Housing p.426, Kinship p.427, Labour p.428, Land p.432, Law p.434, Marriage p.441, Medicine p.443, Missions p.446, Music p.450, Nutrition p.451, Psychology p.452, Race relations p.453, Religion p.465, Rock art p.468, Separatist movements p.471, Social welfare p.472, Urban studies p.474, Women p.475

For other possible subjects, see the asterisked headings in Vol. 1, Section 1

\section{INDEX OF ETHNIC GROUPS AND LANGUAGES \\ 563 INDEX TO SUBJECT-HEADINGS \\ 573 ABBREVIATIONS TO TITLES OF PERIODICALS}




\section{INDEX OF ETHNIC GROUPS}

The names of many ethnic groups have been subordinated to the names of larger groupings (indicated by "See under" references).

There are then 5 types of entry in this index:-

(1) a direct indication of the volume and page-number where entries on a group can be found; thus 2:345 refers to Volume 2, page 345

(2) "See references" from alternative spellings or names

(3) "See also references" when entries for an ethnic group can be found in more than one part of the Cumulative Bibliography

(4) "See under references" from the name of an ethnic group to the name of another group to which it has been subordinated; thus entries about the Ababda will he found incorporated into those about the Beja

(5) "Cp. references" when there is doubt whether two names really are alternatives or not.

Ababda. See under Beja

Ahabua. See: Bua

Ahacama. See: Bachama

Abaha. See: $\mathrm{Ha}$

Abakuria. See: Kuria

AbaMbo. See: Fingo

Abandia. See: Bandia

Abango. See: Bangi

Abasimbete. See: Simbiti

Abdullab. See under Sudan Arab

Abidji or Abiji. See under Anyi

Abouré. See: Abure

Abron. See: Brong

Abudab. See under Sudan Arab

Abukeia. See: Logo-Avokaya

Abure 2:345

Abwor. See: Labwor

Abyssinians. See: Ethiopians

Achikunda. See: Kunda

Acoli or Acholi 3:134

Acwabo. See: Chwabo

Ada. See: Edda

Adangme 2:96

Adara. See: Kadara

Adare. See: Harari
Adele 2:374

Adhola 3:135

Adigo. See: Digo

Adija. See: Bubi

Adja 2:355

Adjao. See: Yao

Adyukru 2:345

Aembu. See: Embu

Afar 1:632

Afitti. See under Nuba

Afo or Afu. See under Idoma

Afusare (= Jarawa) See: Jarawa

Agbari. See: Gbari

Agbor 2:221

Aggiek. See: Dorobo.

Agikuyu. See: Kikuyu

Agiriama or Agiryama. See: Giryama

Agni. See: Anyi

Agolik or Agorok. See: Kagoro

Ait Atman. See: Bella

Aït Baamran 1:494

Aït Bella. See: Bella

Aizi. See under Ebrie

Aïzo 2:355

Aja [Sudan]. See under Kresh 
Aja [West Africa]. See: Adja

Ajaua. See: Yao

Ajiba. See: Murle

Ajukru. See: Adyukru

Akamba. See: Kamba [Kenya]

Akan 2:97 See also Akim; Akwapim; Ashanti; Twi

Akanda. See: Shabe

Akare. See: Kare

Akebu. See: Kebu [Togo]

Akie [Côte d'Ivoire]. See: Akye

Akikuyu. See: Kikuyu

Akim (incl. Akim Abuakwa and Akim

Kotoku) 2:98

Akposso. See: Kposo

Akuapem. See: Akwapim

Akurumba. See: Kurumba

Akwahu. See: Kwahu

Akwamu. See under Twi

Akwapim. See under Twi

Akye 2:345

Akyem. See: Akim

Aladyan or Aladian or Alagian 2:345

Alendu. See: Lendu

Alladian. See: Aladyan

Alomwe. See: Lomwe

Alur 2:595 (incl. Angal; Jukot) 3:135

AmaBaca. See: Baca

AmaMfengu. See: Fingo

Amar. See under Burji-Konso

Amarar. See under Beta

Amaxhosa. See: Xhosa

Amba (and Bwisi) 3:136

Ambala. See: Mbala

Ambaquista. See: Mbaka [Angola]

Ambo (group) 3:505, 3:290. See also: Kwanyama; Ndonga

Ambunda. See: Mbun

Amer. See: Beni Amer

Amhara. See: Ethiopians

Amu 3:92

Anago. See: Nago

Anaguta 2:221

Anam. See under Igbo

Anang. See under Ibibio

Andriana. See: Merina

Angai. See under Bangba

Angal. See under Alur

Angas 2:192

Angba 2:595
Angbandi. See: Ngbandi

Anglo. See under Ewe

Angolares 2:653

Anguru. See: Lomwe

Anlo. See: Anglo

Annang. See: Anang

Ano. See: Tyokossi

Anpika. See: Bolewa

Antaifasy. See: Antefasi

Antaimoro. See: Antemoro

Antaisaka. See: Antesaka

Antakarana. See: Antankarana

Antambahoaka 3:368

Antañala. See: Tañala

Antandroy 3:368

Antankarana 3:368

Antankay. See: Bezanozano

Antanosy 3:368

Antefasi 3:369

Antemoro 3:369

Antesaka 3:369

Antsihanaka. See: Sihanaka

Anuak 1:574

Anufo. See: Tyokossi

Anyi 2:346

Anyuak. See: Anuak

Aonin. See: Topnaar

Apfourou. See: Furu

Apindji. See: Pende

Apoi. See under Ijo

Arabs 1:175

Arbore. See under Burji-Konso

Aregwa. See: Irigwe

Ari [Côte d'Ivoire]. See: Abidji

Ariaal Rendille. See: Rendille

Arimi. See: Rimi

Arma. See under Songhai

Aro [Nigeria]. See under Igbo

Arusha. See under Masai

Arussi or Arusi. See under Galla

(Oromo)

Asante. See: Ashanti

Ase. See: Shabe

Ashanti 2:98

Ashraf. See under Beja

Assaorta. See under Saho

Asu (Wasu, Pare) 3:183

Aten. Cp. Ganawuri

Atetela. See: Tetela

Athu. See: Asu 
Atman. See: Bella

Atshuru. See under Songo

Attiye. See: Akye

Atyap. See: Katab

Atye. See: Akye

Auen. See under Bushman

Aulliminden. See: Iullemmeden

Auni. See under Bushman

Aushi (Ushi) 3:291

Avatime 2:374

Avausi. See: Aushi

Avokaya or Avukaya. See: Logo-Avokaya

Avungara. See: Zande

Awellimiden. See: Iullemmeden

Awerri. See: Itsekiri

Awuna. See under Ewe

Awutu. See: Fetu

Ayizo. See: Aïo

Azande. See: Zande

Azza 2:331

Ba-Temi. See: Sonjo

Baamran. See: Ait Baamran

Baatonu. See: Bariba

Babangi. See: Bangi

Babi 2:507

Babila. See: Bira

Babinga (pygmies) 2:484 (incl. Baka; Ngombe)

Baboma. See: Boma [Zaire]

Baca (amaBaca; Bhaca) 3:494

Bachama or Bacama 2:192

Badia. See: Djia

Badjo. See under Mangbetu

Badjue. See under Maka-Njem

Badyaranke 2:312

Baele. See: Bideyat

Bafia 2:394

Bafourou. See: Furu

BafueNuma. See under Nyali

Bafut. See under Tikar

Bafute. See: Vute

Baga 2:334

Bagam 2:395

Baganda. See: Ganda

Baggara. See under Sudan Arab

Bagirmi 2:492

Bagiuni. See: Bajuni

Bagnoun. See: Banyun

Bai. See under Ndogo-Sere
Baila. See: Ila

Bainuk. See: Banyun

Baja. See: Baya

Bajuni 1:644

Baka. See under (1) Babinga; (2) Pygmy

Bakioko. See: Chokwe

Bakoko 2:395

Bakosi. See: Kosi

Bakunda. See: Kunda

Bakwa Kalonji. See under Luba

Bakwa Longo. See under Lulua

Bakwa Lubo. See under Songye

Bakwa Luntu. See: Luntu

Bakwa Nyembi. See under Lulua

Bakweli or Bakweri or Bakwiri. See: Kpe

Balambo. See: Barambo

Balanta 2:434

Bale. See: Lendu

Balengi 2:651

Balese 2:596

Bali [Cameroun] 2:395

Bali [Zaïre] 2:596. Cp. also Bua

Ballante. See: Balanta

Baloki. See: Boloki

Balom. See under Bafia

Bamana. See: Bambara

Bamangwato. See: Ngwato

Bamba. See under Mbede

Bambara 2.321

Bambuli. See: Mbole

Bambuti. See: Mbuti

Bamileke 2:395

Bamum or Bamoum or Bamun 2:397

Banaka. See under Batanga

Banana-Kolon. See under Masa

Banda 2:498

Bandala 1:575

Bandi 2:425

Bandia or Bandiya 2:596

Bandji. See: Namshi

Bandjo. See: Bondjo

Bane. See under Ewondo

Banen 2:398

Bangangte. See under Bamileke

Bangba 2:596

Bangi 2:585

Bangubangu 2:606

Bangwa or Ngwe. See under Bamileke 
Bangwato. See: Ngwato

Banhum or Banhun. See: Banyun

Banjun. See under Bamileke

Banmana. See: Bambara

Banoho. See under Batanga

Banta. See under Temne

Bantu 1:176

Bantu Kavirondo. See: Luhya

Banun. See: Bamum

Banyang. See: Nyang

Banyin. See: Banen

Banyun 2:434

Banza. See under Ngala [Zaïre]

Banziri. See: Gbanziri

Baoulé. See: Baule

Bapuku. See under Batanga

Baqqara. See: Baggara

Bara 3:369

Barabaig. See under Tatoga

Barabra. See under Nubian

Baraguyu. See under Masai

Barambo 2:596

Barba. See: Bariba

Barbaig. See: Barabaig

Barea. See: Baria

Bargou or Bargu. See: Bariba

Bari [Sudan] 1:575

Baria. See under: Kunama

Bariba 2:355

Barotse. See: Lozi

Bartha. See: Berta

Baruba. See: Bariba

Baruun. See: Burun

Baruwu. See: Bariba

Barwe 3:346

Basa [Cameroun] 2:398

Basa [Liberia]. See: Bassa [Liberia]

Basa-Komo 2:192

Basaa. See: Basa [Cameroun]

Basari [Senegal, Guinée,

Guiné-Bissau] 2:312. See also under Konyagi

Basari [Togo/Ghana]. See: Bassari [Togo/Ghana]

Baseda 2:356

Bashi. See: Shi

Basongo Meno. See under: Songo

Bassa [Cameroun]. See: Basa

[Cameroun]

Bassa [Liberia] (Mani) 2:425
Bassari [Senegal etc.]. See: Basari

Bassari [Togo/Ghana] (Chambi) 2:374

Basuto. See: Sotho (Southern)

Batahin. See under Sudan Arab

Batammaliba or Batammariba. See:

Betammaribe

Batanga 2:398 (incl. Banoho; Bapuku; Banaka)

Batlhokwa. See: Tlokwa

Batonu. See: Bariba

Batswa or Batwa. See: Twa

Baula. See: Brame

Baule 2:346

Baushi. See: Aushi

Bavuma. See: Vuma

Bawule. See: Baule

Baya 2:499. See also Manja

Bayele (Pygmies) 2:652

Baynunk. See: Banyun

Bazantche 2:356

Bdon. See under Bobo

Bechuana. See: Tswana

Bedawiye. See: Beja

Bedik 2:312

Bedja. See: Beja

Bedu 2:101

Beena Luluwa. See: Lulua

Bega [Sudan]. See: Beja

Beir. See: Murle

Beja 1:563

Bekoe. See: Bayele

Bekpak. See: Bafia

Bekpokpam. See: Konkomba

Bekuin. See: Bayele

Belanda (Bor or Mberidi). See under Lwo

Belanda (Bviri or Mbegumba). See under Ndogo-Sere

Belen. See: Bilin

Beli. See: Bideyat

Beliyan. See: Basari

Bella. See under Tuareg

Belle 2:425

Bemba 3:291 (incl. Ngoma; but see also: Ngoma)

Bembe 2:507, 2:606

Bena 3:183. See also Hehe

Bena Bayashi. See under Songye

Bena Ebombo. See under Songye

Bena Kanyoka. See: Kanyoka 
Bena Kayumba. See under Hemba

Bena Lulua. See: Lulua

Bena Mai 2:640

Bena Mitumba. See: Mitumba

Bena Muhona 2:630

Bena Ngoma. See: Ngoma

Bena Ngungi. See under Songye

Bena Nkuvu. See: Nkuvu

Bende 3:184

Benga 2:652

Beni Amer. See under Beja

Beni M'zah. See: Mzab

Beni Shangul. See: Berta

Benialenge. See: Bira

Benin 2:221

Beo. See under Angba

Berba. See: Bariba

Berber 1:461, 1:488, 1:495, 1:502,

1:519. See also: Kabyle

Bergdama 3:506

Beri. See under Anuak

Berom. See: Birom

Berri. See: Beri

Berta (Beni Shangut) 1:601

Berti 1:565

Besanin. See: Bisharin

Besorube. See under Somba

Beta Israel. See: Falasha

Betammaribe. See under Somba

Bete 2:347 (incl. Godye)

Beti 2:398

Betsileo 3:370

Betsimisaraka 3:370

Bezanozano 3:370

Bhaca. See: Baca

Bi-Tchambé. See: Bassari

[Togo/Ghana]

Biafada 2:435

Bicharin. See: Bisharin

Bideyat 2:492

Bidyogo 2:435

Biin Kanyôk. See: Kanyoka

Bijago. See: Bidyogo

Bikele. See under Maka-Njem

Bila. See: Bira

Bilala. See under Bagirmi

Bilen. See: Bilin

Bilin 1:632

Bille 2:192

Bimbia. See: Subu
Bindi or Bindji. See: Mbagani

Binga. See: Babinga

Bini (Edo). See under Benin

Binja. See: Zimba [Zaïre]

Binji. See: Mbagani

Bira (incl. Lengola) 2:597

Birifor 2:362

Birom 2:192

Birwa 3:477

Bisa [West Africa] (Busanse) 2:362. See also: Busa

Bisa [Zambia] 3:293 (For Bisa jointly with Bemba, see: Bemba)

Bisago. See: Bidyogo

Bisano. See: Bisa [West Africa]

Bisharin. See under Beja

Bissago. See: Bidyogo

Biyobe (= Besorube). See under Somba

Boa [Zaïre]. See: Bali [Zaïre]

Bobangi. See: Bangi

Bobo (Bobo-Fing and Bobo-Ule) 2:362 (incl. Bwa; Bdon; Bwaba.

For Bobo-Dyula see: Dyula)

Bofi. See under Baya

Bogos. See: Bilin

Bole [Nigeria]. See: Bolewa

Bole [Zaïre]. See: Mbole

Bolewa 2: 192

Bolia. See under Ntomba

Boloki or Boluki 2:585

Boma [Congo Republic]. See under Teke

Boma [Zaïre] (Buma) 2:611

Bomitaba 2:507

Bomvana 3:494

Bomwali (Sangha-Sangha) 2:507

Bondei 3:184

Bondjo 2:507

Bondongo 2:507

Bongo-Baka (group) 1:575

Boni: 1:644

Bono. See: Brong

Boobe. See: Bubi

Booran. See: Boran

Bor. See: Belanda

Boran or Borana. See under Galla (Oromo)

Borgawa. See: Bariba

Bornu. See: Kanuri 
Boro. See: Shinasha

Bororo. See: Fulbe

Bouendi. See: Bwende

Boulou. See: Bulu

Boulsi. See: Bulsa

Boussa. See: Busa

Boussancé. See: Bisa [West Africa]

Bouzou. See: Buzu

Bowiri 2:375

Bozo (Sorko, Sorkawa) 2:322

Brakna 2:308

Brame 2:435

Bron. See: Brong

Brong (Bono) 2:101 (incl. Nkwanta); $2: 348$

Bua 2:597. Cp. also: Bali [Zaïre]

Bubi 2:653

Budja 2:585

Buduma 2:492

Budya 3:318

Builsa. See: Bulsa

Buja. See: Budya

Bujeba 2:652

Bukusu. See under Gisu

Bulanta. See: Balanta

Bullom. See: Bulom

Bulom 2:68

Bulsa 2:102

Bulu 2:398 (For Beti jointly with Bulu see: Beti)

Bum. See: Mbum

Buma. See: Boma [Zaïre]

Bunda. See: Mbun

Bungo 2:640

Bungu. See under Nyamwezi

Bura [Nigeria] 2:192

Burji-Konso (group) 1:601

Burun 1:576

Busa 2:192

Busanse. See: Bisa [West Africa]

Bushi. See: Shi

Bushman 3:413, 3:516 (incl. Auen, $\neq$ Auni, G/wi, $\neq$ Khomani, Kxoe, Naron). See also: Bushman, Khoisan, Kung, Kwe, Sarwa)

Bushongo (Kuba) 2:621 (incl. Nyongo)

Bussa. See: Busa

Bussansi. See: Bisa [West Africa]

Bute. See: Vute
Buye 2:606

Buzi. See: Lomna

Buzu 2:331

Bviri. See: Belanda

Bwa. See under Bobo

Bwaba. See under Bobo

Bwaka. See: Nghaka [Zaïre]

Bwende 2:507

Bwisi. See under Amba

Bworo. See: Shinasha

Cabinda. See under Vili

Caga. See: Chaga

Cakosi. See: Tyokossi

Calabari. See: Kalabari

Cama. See: Ebrie

Camant. See: Kemant

Camus. See: Njemps

Cewa 3:336

Chaga 3:184

Chagga. See: Chaga

Chaha. See under Gurage

Chakosi. See: Tyokossi

Chamba [Togo/Ghana]. See: Bassari

[Togo/Ghana]

Chambi. See: Bassari [Togo/Ghana]

Chamus. See: Njemps

Chaouïa. See: Shawiya

Chewa. See: Cewa

Chibuk. See under Margi

Chiga. See: Kiga

Chikunda. See: Kunda

Chirima. See under Makua

Chishinga. See under Bemba

Chokosi. See: Tyokossi

Chokwe 2:640

Chompus. See: Njemps

Chopi 3:346

Chuabo. See: Chwabo

Chwabo 3:347

Chwana. See: Tswana

Ciga. See: Kiga

Cikunda. See: Kunda

Cokwe. See: Chokwe

Colo. See: Shilluk

Coloured 3:415

Congo. See: Kongo

Coniagui. See: Konyagi

Cotocoli. See: Tem

Cuabo. See: Chwabo 
Cuambo. See: Chwabo

Cunama. See: Kunama

Dabosa. See: Toposa

Dadjo. See: Dago

Dagara. See: Dagari

Dagari 2:102 (incl. Wiili or Wule or Ule)

Dagbamba. See: Dagomba

Dago. 1:565

Dagomba (Dagbamba etc.) 2:102

Dagu. See: Dago

Dahomean. See: Fon

Dai 2:492

Dair. See: Afitti

Daju. See: Dago

Dakakari or Dakkakari 2:192

Dan 2:425

Danakil. See: Afar

Dangbe or Dangme. See: Adangme

Danxome. See: Fon

Darassa. See under Sidama

Dasa. See under Nago

Dassanetch or Dathanik. See: Geleba

Datoga. See. Tatoga

Daze [Chad]. See under Teda

Dciriku. See: Diriku

Dembu. See: Ndembu

Dendi 2:356

Denkawi. See: Dinka

Denkyira 2:103

Derasa. See: Darassa

Diakhanke. See: Dyakanke

Dialonke. See: Dyalonke

Dian. See: Dyan

Diawara. See: Dyawara

Dida 2:348

Didinga 1:576

Dieli. See under Senufo

Digo. See under Nyika

Dilling. See under Nuba

Dime 1:602

Ding. See: Dzing

Dinka 1:576

Diola. See: Dyola

Dioula. See: Dyula

Diriku 2:642

Djaga. See: Chaga

Djao. See: Yao

Dje. See: Holli
Djem. See: Maka-Njem

Djerma. See: Dyerma

Djia 2:611

Djompra or Djumperi. See: Kutep

Doado. See: Doayo

Doayo. See under Namchi

Dodinga. See: Didinga

Dodos or Dodoth. See under Karamojong

Doe. See under Zaramo

Doghosie 2:363

Dogon (Habe) 2:322

Doko 2:585

Dompago. See: Logba

Dondo 2:507

Dongo. See under Kresh

Dongotono. See under Lango [Sudan]

Dorobo (Okiek) 3:93

Douala. See: Duala

Dowatyayo or Dowayayo or Doyayo. See: Doayo

Dschagga. See: Chaga

Duala 2:399

Dubab. See under Nuba

Dukunz 1:602

Duma [Gabon] 2:502

Duma [Rhodesia/Zimbabwe] 3:318

Dumbuseya. See under Shona

Duru 2:400

Duruma. See under Nyika

Dyakanke 2:3 12

Dyalonke (Yalunka) 2:334

Dyan 2:363

Dyawambe 2:325

Dyawara 2:325

Dyerma 2:331

Dyola 2:312

Dyula 2:348

Dzalamo. See: Zaramo

Dzem. See: Maka-Njem

Dzindza. See: Zinza

Dzing 2:611

Eastern Iullemmeden (Eastern

Iwellemmeddän). See under Tuareg

Ebira. See: Igbira

Ebrie 2:349

Edda. See under Igbo

Ediye. See: Bubi 
Edo. See: Bini [Edo]

Efe. See under Pygmy

Effutu. See: Fetu

Efik 2:223

Egba. See under Yoruba

Eghap. See: Bagam

Egun. See: Gun

Ekoi 2:224

Ekonda. (1) See: Konda; (2) See under Nkundo

Ekondja. See: Djia

Ekot Ngba 2:400

Elgeyo. See: Keyo

Elgonyi. See: Kony

Eljemasi. See: Njemps

Elmolo 3:93

Embu 3:93

Engassana. See: Ingassana

Enjemasi. See: Njemps

Ennemor. See under Gurage

Enya 2:597

Erbore. See: Arbore

Ergeibat. See: Reguibat

Erokh. See: Iraqw

Esan. See: Ishan

Esimbi 2:400

Ethiopian Jews. See: Falasha

Ethiopians 1:602

Eton. See under Tikar

Evé. See: Ewe

Evuzok. See under Ewondo

Ewe 2:375

Ewondo 2:400

Falascià. See: Falasha

Falasha 1:603

Fali 2:401

Fang 2:502 (incl. Ntum); 2:652

Fante 2:103 See also Twi

Fari. See: Beri

Felupe (Felup). See under Dyola

Feroge (group) 1:578

Fetu 2:104

Fia. See: Bafia

Fika. See: Bolewa

Fingo 3:494

Fiote. See: Vili

Fipa 3:186

Floup. See: Felupe

Fö. See: Fon
Fokeng 3:522

Foma. See under Mbole

Fon 2:356

Foodo. See: Bazantche

For. See: Fur

Fori or Föri. See: Beri

Foto. See: Poto

Fourou. See: Furu

Fula. See: Fulbe

Fulakunda. See under Fulbe

Fulani. See: Fulbe

Fulbe (Fula, Peul, Fulani, Bororo,

Peul, Tukulor) 2:26, 2:193, 2:325.

2:331 (incl. Wodaabe), 2:334 (incl.

Fulakunda Tukulor), 2:401

Fung 1:578

Fur 1:565

Furu 2:585

Fut. See: Bafut

Ga 2:104 (incl. Teshi)

Gade 2:194

Gafat 1:604

Gagu or Gagou 2:349

Gai-Khan. See under Nama

Galab. See: Geleba

Galila. See under Gurage

Galla (Oromo) 1:604

Gallinas. See: Vai

Galuba. See: Geleba

Gâmk. See: Ingassana

Gamuia. See under Sudan Arab

Gan [Burkina Faso]. See under

Lobi

Gananwa. See: Malaboch

Ganawuri 2:194

Ganda 3:136

Ganguela. See. Ngangela

Ganjoola. See: Biafada

Garamantes 1:520

Gbã or Gban. See: Gagu

Gbande. See: Bandi

Gbanziri or Gbanzili 2:499

Gbari 2:194

Gbasa. See: Bassa [Liberia]

Gbaya. See: Baya

Gbaya. See: Kresh

Gbazantche. See: Bazantche

Gbeya. See: Baya

Gbira. See: Bira 
Gciriku. See: Diriku

Ge. See: Mina

Gedeo. See: Darassa

Gekoyo. See: Kikuyu

Geleba. See under Burji-Konso

Gelli. See: Bayele

Gen. See: Mina

Gengele. See: Ngengele

Gere 2:349. See also: Kran

Gerzé 2:337. See also Kpelle

Geshu. See: Gisu

Gesogo or Gesoho. See: Poke

Gesu. See: Gisu

Geyo. See: Keyo

Ghimirra. See: Gimira-Maji (group)

Gidar 2:402

Gikuyu. See: Kikuyu

Gimira-Maji (group) 1:607

Giryama or Giriama. See under Nyika

Gishu. See: Gisu

Gisiga 2:402

Gisu 3:140

Giziga. See: Gisiga

Glidyi. See under Ewe

Goba. See under Shona

Godye. See under Bete

Goffa. See: Kafa

Gogo 3:187

Goin. See: Guin

Gola [Liberia] 2:425

Golo 1:578

Gombe. See: Ngombe

Gonga. See: Kafa

Gonja [Ghana]. See: Guang

Gora. See: Gola [Liberia]

Goran. See under Teda

Goroa. See under Iraqw

Gorongozi. See under Teve

Goula. See: Gula

Goun. See: Gun

Gouraghe. See: Gurage

Gourma. See: Gurma

Gouro. See: Guro

Gourounsi. See: Grusi

Gova. See: Goba

Grebo. See under Kru

Grikwa or Griqua. See under Hottentot

Grusi or Grunshi 2:363
Guang 2:105

Guen. See: Mina

Guéré. See: Gere

Guerzé. See: Gerzé

Guidar. See: Gidar

Guin. See under Turuka

Guissiga. See: Gisiga

Gula or Gulla [Chad] 2:492

Gulmance. See: Gurma

Gumba (legendary) 3:93

Gumuz or Gumus 1:607

Gun 2:357. Cp. also: Popo

Gunza. See: Gumuz

Gurage 1:607 (incl. Ennemor)

Gurma or Gurmantche 2:363

Guro 2:349

Gurunsi. See: Grusi

Gusii 3:93

Guum. See: Humbu

Gwaka. See: Ngbaka [Zaïre]

Gwali or Gwari. See: Gbari

Gwe [Kenya]. See under Luhya

Gwi. See: Guin

Gyaman. See: Brong

Gyeli. See: Bayele

Gyula. See: Dyula

Gyuman. See: Ebrie

Ha 3:187

Haalpulaar. See: Tukulor

Habab 1:632

Habe [Mali]. See: Dogon

Habe [Nigeria]. See: Shabe

Haddad 2:492

Hadendowa. See under Beja

Hadimu 3:225

Hadiya 1:608

Hadjerai 2:492

Hadzapi or Hadza 3:188

Hahm. See: Jaba

Hain/lum or Haiom. See: Heikum

Hal Pular. See: Tukulor

Halawin. See under Sudan Arab

Halenga. See under Beja

Halphoolaren. See: Tukulor

Ham. See: Jaba

Hamar [Ethiopia]. See: Amar

Hamba 2:622

Hamitic 1:176

Hamran. See under Sudan Arab 
Hananwa. See: Malaboch

Hanga. See under Luhya

Hangiro. See under Haya

Hanya 2:642

Haoussa. See: Hausa

Harari (inhabitants of Harar city) 1:608

Harbora. See: Arbore

Hassanya 2:308

Hausa 2:194

Hawawir. See under Nubian

Haya 3: 188

Hehe (group) 3:189. See also Bena

Heikum. See under Kung

Hema. See: Hima

Hemba 2:630

Hendawa. See: Hadendowa

Henga. See under Tumbuka

Hera (and Njanja) 3:318

Herero 3:506

Higi or Hiji 2:402

'Hill' Nubian. See under Nuba

Hima (Hema, Huma) 2:597. See also:

Nkole. See also under: Tutsi

Hindo. See: Basongo Meno

Hlubi 3:494

Holli or Hollidje. See under Nago

Holo 2:611

Holoholo 2:631

Hom. See: Kom

Homr. See under Sudan Arab

Horohoro. See: Holoholo

Hororo (Mpororo) 3:140

Hottentot 3:495 (incl. Grikwa. See also: Khoisan; Korana; Nama)

Houeda. See: Peda

Hova (=Merina). See: Merina

Hukwe Bushmen. See: Kwe

Huma. See: Hima

Humbe. See under Nyaneka

Humbu 2:611

Humr. See: Homr

Hunde 2:606

Hungu 2:642

Hungwe. See: Ungwe

Hurutse 3:477

Hweda. See: Peda

Hyam. See: Jaba

Hyapa. See: Shabe
Iacca. See: Yaka

Ibibio 2:224. See also Efik

Ibo. See: Igbo

Idoma (incl. Afo) 2:199

Ife. See under Yoruba

Igala or Igara 2:199

Igbira or Igbiri 2:200

Igbo $2: 225$

Ijaw. See: Ijo

Ijebu. See under Yoruba

Ijo 2.230. See also Kalabari

Ik. See under Karamojong

Ika. See under Igbo

Ikizu. See under Kwaya

Il Chamus or Il Jamus or Il Tiamus.

See: Njemps

Il Torobo. See: Dorobo

Ila 3:294

Ilamba. See: Nilyamba

Ilparakuyo. See: Baraguyu

Iltiamus. See: Njemps

Imazighen. See: Berber

Imraguen 2:308

Indian 1:177, 3:22. 3.417

Indri. See under Feroge

Ingassana or Ingessana 1:578

Ingwe. See: Ungwe

Inor or Innemor. See: Ennemor

Ioullemmeden. See: Iullemmeden

Ipunu. See: Punu

Iramba. See: Nilyamba

Irangi. See: Langi

Iraqw 3:191

Irbore. See: Arbore

Irigwe 2:200

Isanzu. See: Nilyamba:

Isekiri. See: Itsekiri

Ishan. See under Benin

Isoko. See under Sobo

Isongo. See: Mbati

Isubu or Isuwu. See: Subu

Iteso. See: Teso

Itsekiri 2:231

Iullemmeden. See under Tuareg

Iwa. See under: Mwanga

Iwellemmeddän or Iwullemmeden.

See: Iullemmeden

Iyembe. See: Yembe

Izon. See: Ijo 
Jaba 2:200

Jabo. See under Kru

Jaca or Jaga. See: Yaka

Jakhanke. See: Dyakanke

Jalonke. See: Dyalonke

Jang. See: Dinka

Janjero 1:608

Jao. See: Yao

Jarawa 2:200

Jaunde. See: Ewondo

Jawunda 3:318

Jekri. See: Itsekiri

Jeli. See: Dieli

Jerawa. See: Jarawa

Jews 1:177. See also: Falasha

Jie. See under Karamojong

Jieng. See: Dinka

Jita. See: Kwaya

JoAbwor. See: Labwor

Jola. See: Dyola

Jompre. See: Kutep

Jomvu. See under Nyika

Joola. See: Dyola

Jukot. See under Alur

Jukun 2:200

Jula. See: Dyula

Jur. See under Lwo

Juula. See: Dyula

Kaa. See under Rolong

Kãa or Kãaba. See: Gan [Burkina Faso]

Kababish. See under Sudan Arab

Kabinda. See: Cabinda

Kabiye. See: Kabre

Kabre or Kabye 2:378

Kabyle 1:503

Kadara 2:200

Kafa 1:608

Kaffa or Kafficho or Kaffitsho. See: Kafa

Kagoro [Nigeria] 2:200

Kaguru 3:191

Kahonde. See: Kaonde

Kaka 2:204

Kakanda. See: Shabe

Kakonga. See: Konga

Kakongo. See under Vili

Kakwa 1:578

Kalabari 2:231
Kalahari. See: Kgalagadi

Kalanga or Kalana 3:318

Kalenjin. See under Nilo-Hamitic

Kaleri 2:201

Kamanga. See under Tumbuka

Kamba [Congo Republic] 2:507

Kamba [Kenya] 3:94 (For Kamba jointly with Kikuyu, see Kikuyu)

Kanembu 2:493

Kangeju. See: Hadzapi

Kango 2:597

Kanuri 2:201

Kanya 3:485

Kanyoka 2:622

Kaonde 3:294

Kapsiki. See: Higi

Kara [Sudan]. See under Bongo-Baka

Kara [Tanzania]. See under Kerewe

Karamojong 3:140

Karanga 3:319. See also Duma [Zimbabwe]; Mari; Rozwi

Kare (Akare) 2:597

Karimojong. See: Karamojong

Kasena 2:364

Kasonke. See: Khasonke

Kassanga. See under Banyun

Katab 2:202

!Kau. See under Kung

Kazama. See under Kung

Kazibati 2:597

Kebu [Togo] (Akebu) 2:379

Kebu [Zaïre] (Ndo) 2:598

Kede. See under: Nupe. Cp. also Gade

Kefisho. See: Kafa

Keiyo. See. Keyo

Kel Iullemmeden. See: Iullemmeden

Kela 2:622

Kele 2:598

Kemant 1:608

Kenyi or Kene 3:141

Kerewe or Kerebe 3:193

Kete 2:622

Ketu. See under Nago

Keyo or Keyu 3:96

Kgalagadi 3:517

Kgatla 3:517

Khaha. See under Sotho (North)

Khalahadi. See: Kgalagadi

Khasonke 2:325 
Khawalda. See under Sudan Arab

Khoi. See: Hottentot

Khoi-Khoi. See: Nama

Khoisan (Bushman and Hottentot)

3:418. See also separately:

Bushman; Hottentot

Khwakhwa 3:522

Kiga 3:141

Kikuyu 3:96

Kimbu. See under Nyamwezi

Kindiga. See: Hadzapi

Kinga. See under Nyakyusa

Kioko. See: Chokwe

Kipsigis 3:99

Kisama. See: Sama

Kisi [Guinée] 2:337

Kisi [Tanzania]. See under Hehe

Kisii. See: Gusii

Kisonko or Kisongo. See under Masai

Kitara. See: Nyoro

!Ko. See under Kung

Kodo. See under Bongo-Baka

Koena. See: Kwena

Kofyar or Koffiyar 2:202

Koki 3:142

Koko. See: Bakoko

Kololo. See: Lozi

Kom. See under Tikar

Koma [Ethiopia] 1:608

Komba. See: Konkomba

Kombe [Guinea Ecuatorial] 2:653

Komo. See: Kuma

Konda (Ekonda) 2:611

Konde. See: Nyakyusa

Kondjo. See: Konjo

Konga 2:642

Kongo 2:449, 2:507, 2:611 (incl. Mbata; Tsamba)

Konjo 3:142

Konkomba 2:106

Kono 2:68, 2:338

Konongo. See under Nyamwezi

Konso. See: Burji-Konso

Kony 3:100

Konyagi (Coniagui) with Basari

2:338. See also: Basari [Sénégal,

Guinée, Guiné-Bissau] 2:312

Konzo. See: Konjo

Kooroko. See under Bambara

!Kora. See: Korana
Korana 3:496

Koranko 2:68

Koranna. See: Korana

Korekore 320

Kororofawa. See: Jukun

Kosi or Kossi 2:402

Kota 2:504

Kotoko 2:402

Kotokoli. See: Tem

Koukouya. See: Kukuya

Koulango. See: Kulango

Kouroumba. See: Kurumba

Kouyou or Kovo. See: Kuyu

Kpe 2:403

Kpelle 2:426. See also Gerze

Kposo 2:379

Krachi. See under Guang

Krahn. See: Kran

Kran. See under Kru

Kresh 1:578

Krio 2:69

Krobo. See under Adangme

Kroumen. See: Kru

Kru 2:426

Kuanyamna. See: Kwanyama

Kuba [Zaïre]. See under Mboshi. See also: Bushongo

Kubbabish. See: Kababish

Kuku 1:579

Kukuya or Kukwa. See under Teke

Kulango 2:350

Kulere. See: Kaleri

Kulia. See: Kuria

Kulu 2:202

Kulya. See: Kuria

Kumam 3:142

Kumbi. See: Humbe

Kumu 2:598

Kunama (and Baria) 1:632

Kunante 2:435

Kunda (Bakunda) 2:631, 3:337

Kundu 2:403

Kung (Bushman) 2:642 (incl.

Kazama; Kwadi; Kwankala;

Sekwele), 3:507 (incl. Heikum;

!Kau; !Ko; !X $\widetilde{u})$

Kunta (nomads) 2:326

Kunya. See: Nkonya

Kuranko. See: Koranko

Kuria 3:193 
Kurtey 2:331

Kurumba 2:364

Kusasi or Kusae 2:106

Kuta. See: Kota

Kutep 2:202

Kutu [Tanzania] 3:193

Kuyu. See under Mboshi

Kwadi. See under Kung

Kwahu 2:106

Kwala. See under Mboshi

Kwankala. See under Kung

Kwanyama 2:643, 3:508 (Kwanyama Ambo)

Kwavi. See: Masai

Kwawu. See: Kwahu

Kwaya 3:193

Kwayaribe. See: Yoabu

Kwe 3:518

Kwele. See: Kpe

Kwena 3:518

Kwere. See under Zaramo

Kxalaxadi. See: Kgalagadi

Kxatla. See: Kgatla

Kyadya. See: Gade

Kyama. See: Ebrie

Kyedye. See: Kede. Cp. also: Gade

Kyenga. See under Busa

Kyerepong. See under Guang

Laadi. See: Lari

Labwor (and Nyakwai) 3:142

Ladi. See: Leri

Lala [Nigeria] 2:202

Lala [Zambia] 3:294

Lali. See under Teke

Lalia 2:585

Lamba [Zambia] 3:295

Langba. See under Bande

Langi [Tanzania] 3:193

Lango [Sudan] 1:579 (incl. Dongotono)

Lango [Uganda] 3:143

Langulu. See under Sanye

Lari 2:508

Larim. See: Longarim

Larteh. See under Guang

Latuka. See: Lotuko

Lebu 2:314

Lefana 2:379

Lega 2:606
Leku. See under Ngala [Zaïre]

L'ela. See: Lyela

Lele [Zaïre] (Bashi) 2:623. See also: Shi

Lelemi. See: Lefana

Lemba [South Africa] 3:477

Lembutu. See: Rembutu

Lendu 2:598

Lenge. See: Chopi

Lengola. See under Bira

Lengora. See: Lengola

Lepa. See: Lemba

Lesa. See: Sakata

Lese. See: Balese

Letsoalo. See under Sotho (North)

Li. See: Bali [Cameroun]

Lilse. See: Lyela

Limba [Sierra Leone] 2:69

Llogoori. See: Logoli

Loama. See: Loma

Loango Ngoyo. See under Vili

Lobaye. See: Ngbaka [République Centrafricaine]

Lobedu 3:478

Lobi 2:365

LoDagaba. See: Dagari

Logba 2:358

Logbara. See: Lugbara

Logo-Avokaya 2:598

Logoli. See under Gusii

Loikop. See: Samburu

Lokele. See: Kele

Loko. See under Mende

Lokoro. See under Anuak

Loma 2:427

Lomotwa 2:631

Lomwe 3:348

Longarim 1:579

Lonso. See under Yaka

Losso. See under Kabre

Lotuko or Lotuho or Lotuxo 1:579

Lovale. See: Luvale

Lovedu. See: Lobedu

Lozi 3:295

Lozwi. See: Rozwi

Luba (Luba-Lulua) 2:623 (incl.

Bakwa Kalonji), 2:631 (incl.

Samba)

Luba-Hemba. See: Hemba

Lubedo. See: Lobedu 
Luchazi 2:643

Lugbara 3:143

Lugooli. See: Logoli

Luguru 3:193

Luhya 3:100

Luimbi 2:643

Lukpa. See: Logba

Lulua or Luluwa 2:624

Lumbu. See under Punu

Lumbwa. See: Kipsigis

Lunda 2:633

Lunda (of Kazembe) 3:298. See also: Luvale

Lunda-Lovele. See: Luvale

Luntu 2:624

Luo 3:101. See also: Lwo

Lur. See: Alur

Luvale 3:299. See also: Lwena

Luyana. See: Lozi

Luyia. See: Luhye

Lwalwa 2:624

Lwana. See under Nyamwezi

Lwena 2:643

Lwimbi. See: Luimbi

Lwo (group) 1:579 (incl. Belanda or Bor or Mberidi; Jur or Shilluk Luo; Manangeer). See also: Luo

Lyela (L'ela) 2:365

Ma. See: Mano

Maasai. See: Masai

Maban. See under Burun

Maconde. See: Makonde

Macua. See: Makua

Mada 2:202

Mafa. See under Matakam

Maganja. See: Manganja

Magi. See: Gimira-Mali (group)

Mahafaly 3:371

Mahamid 2:493

Mahas. See under Nubian

Mahe. See: Mahi

Mahi 2:358

Maiaca. See: Yaka

Majangir. See: Masongo

Maka-Njem 2:403

Makanya. See: Kanya

Makhuwa. See: Makua

Makonde 3:348

Makua. 3:349
Makwa. See under Mboshi

Malaboch. See under Sotho (North)

Malawi. See: Maravi

Malete 3:518

Malinke 2:338. See also: Mande

Malozi. See: Lozi

Mambila 2:404

Mambwe 3:300

Mamluk. See under Sudan Arab

Mamvu-Mangutu 2:599

Manangeer. See under Lwo

Mancanha. See: Brame

Mandara 2:404

Mandari. See: Mondari

Mande or Manding 2:27. See also Malinke

Mandingo or Mandinka. See: Malinke

Mandja or Mandjia. See: Manja

Mandyak 2:435

Manganja. See under Nyanja

Mangati. See under Tatoga

Mangaya. See under Feroge

Mangbetu 2:599

Mangia. See: Manja

Mani. See: Bassa [Liberia]

Maninka. See: Malinke

Manja 2:499. See also Baya

Manjaco. See: Mandyak

Mankanya. See: Brame

Mano 2:427

Manyika 3:320

Manze. See: Manja

Mao 1:609

Maouri. See: Mawri

Mapopoi. See: Popoi

Maragole. See: Logoli

Maraka. See: Marka

Maravi 3:244

Marba. See under Masa

Margi or Marghi 2:202

Mari 3:321

Marka 2366

Marofotsy 3:371

Masa 2:404

Masai 3:194 (incl. Arusha; Baraguyu;

Kisonko), 3:102 (incl. Niemps)

Masarwa. See: Sarwa

Masemola. See under Sotho (North)

Mashukolumbwe. See: Ila

Masongo [Ethiopia] 1:609 
Massa. See: Masa

Massai. See: Masai

Matabele. See: Ndebele

Matakam 2:404

Matengo 3:195

Matshaga. See under Mangbetu

Matumbi 3:196

Mauri. See: Mawri

Mawri 2:331

Maxi. See: Mahi

Mbagani 2:624

Mbaka [Angola] (Ambaquista). See under Mbundu(ki)

Mbaka [Zaïre]. See: Ngbaka [Zaïre]

Mbala 2:613

Mbali [Angola] 2:643

Mbangala. See under Mbundu(ki)

Mbangi. See: Bangi

Mbangubangu. See: Bangubangu

Mbati 2:499

Mbede 2:504

Mbegumba. See: Belanda

Mbem. See under Tikar

Mbembe 2:232

Mberidi. See: Belanda

Mbesa 2:599

Mbire 3:321

Mbole 2:599 (incl. Foma), 2:624

Mboshi or Mbosi (group) 2:508

Mboum. See: Mbum

Mbugu [Tanzania] 3:196

Mbugwe 3:196

Mbukushu 3:518

Mbum 2:405

Mbun or Mbunda 2:613

Mbundu(ki) 2:643 (incl. Mbaka (Ambaquista); Mbangala; Ngola)

Mbundu(u) 2:644 (incl. Sambu; Wambu)

Mbuti (Bambuti) (pygmies) 2:600. See also: Efe; Pygmy

Mbuza. See: Budya

Mbwela [Angola]. See: Ngangela

Mbwela [Zaïre]. See: Nkoya

Meban. See: Maban

Medje. See under Mangbetu

Meidob. See: Midob

Meje. See: Medje

Mende 2:70

Meno. See: Basongo Meno
Mensa 1:633

Merina 3:371

Meroitic 1:565

Meru [Kenya] 3:103

Merule. See: Murle

Mesongo. See: Masongo

Messiria. See under Sudan Arab

Metu. See under Moru-Madi

Mfantra. See: Nafana

Mfengu. See: Fingo

Mfinu 2:614

Mfiote. See: Vili

Mfute. See: Vute

Midob. See under Nubian

Miisiirii. See: Messiria

Milembwe 2:625

Mileri. See: Messiria

Mina. See under Ewe

Minianka or Minyanka. See under

Senufo

Mino. See: Basongo Meno

Misiiriiya. See: Messiria

Mituku 2:602

Mitumba 2:634

Mizab. See: Mzab

Moba [Toga] 2:379

Mofu 2:405

Mole. See: Mossi

Momfou or Momvu. See:

Mamvu-Mangutu

Mondari 1:580

Mongo [Zaïre] ('Lolo')

(Nkundo-Mongo) 2:585. See also Nkundo

Monjombo or Monzombo 2:508

Moose. See: Mossi

Morokodu. See under Bongo-Baka

Moropom. See under Karamojong

Moru-Kodo. See under Bongo-Baka

Moru-Madi (group) .1:580

Mossi or Moshi 2:366

Mossilongi. See: Solongo

Moundang. See: Mundang

Mourle. See: Murle

Mozabites. See: Mzab

Mpama 2:586

Mpangwe. See: Fang

Mpondo 3:496

Mpororo. See: Hororo

Mrima. See under Nyika 
Muktele. See under Matakam

Mum. See: Bamum

Mumvu. See: Mamvu-Mangutu

Mundang 2:493

Mundari. See: Mandari

Munshi. See: Tiv

Murle or Murule 1:581

Musgu 2:405

Mushirongo or Musserongo or Muxilongo. See: Solongo

Mwaba. See: Moba

Mwanga (and Iwa) 3:196

Mwani. See under Haya

Mwera or Mwela 3:196

Mwila. See under Nyaneka

Mwimbi. See under Meru

Myene 2:504

Mzab 1:503

N'buti. See: Mbuti

Naath. See: Nuer

Nafana 2:106

Nago (Yoruba peoples in Dahomey) 2:358

Naguta. See: Anaguta

Nalou. See: Nalu

Nalu 2:340

Nama (Khoi-Khoi, Namakwa, Naman, Nama-Hottentot) 3:508. See also: Khoisan, Hottentot

Nama-Hottentot or Namakwa or Naman or Namaqua. See: Nama

Namchi or Namji or Namshi or Namtchi 2:405

Namwanga. See: Mwanga

Nande 2:607

Nandi 3:104

Nanzwa 3:321

Nath. See: Nuer

Natioro. See under Wala

Naudeba 2:379

Ndaka. See under Nyali

Ndamba 3:196

Ndau 3:349

Ndebele 3:321

Ndembu or Ndembo 3:300

Ndendeuli. See under Ngoni

Nderebo. See: Dorobo

Ndiki. See under Banen

Ndjem. See: Maka-Njem
Ndjevi. See: Nzabi

Ndo. See: Kebu [Zaïre]

Ndogo-Sere (group) 1:581 (incl.

Belanda or Bviri or Mbegumba)

Ndonga 3:509

Ndorobo. See: Dorobo

Ndout or Ndut. See under Serer

Ndzabi. See: Nzabi

Ndzawu. See: Ndau

Ndzundza 3:479

Nei ti naadh. See: Nuer

Nemadi 2:308

Nen. See: Banen

Nfumu. See under Mfinu

Nfunungu. See: Mfinu

Ngala [Angola]. See: Ngola

Ngala [Sudan] 1:581

Ngala [Zaïre] 2:586

Ngangela (Mbwela) 2:645

Ngangulu or Ngangoulou. See under Teke

Ngara. See: Ngala [Sudan]

Ngasa 3:196

Ngata. See under Nkundo

Ngbaka [République Centrafricaine] 2:499

Ngbaka [Zaïre] 2:587

Ngbandi 2:588

Ngelima. See under Angba

Ngengele 2:607

Ngere. See: Gere

Ngindo 3:196

Ngola. See under Mbundu(ki)

Ngoma 2:634. See also under Bemba

Ngombe 2:588. See also under Babinga

Ngonde. See: Nyakyusa

Ngoni (and Ndendeuli) 3:196, 3:300, 3:337

Ngulu [Tanzania] 3:197

Ngumba. See under Maka-Njem

Ngunda. See under Nbandi

Ngungulu or Ngungwel or Ngungwoni. See: Ngangulu

Nguni 3:418

Nguru [Malawi] 3:338. See also: Lomwe; Makua

Nguru [Tanzania] or Nguu. See: Ngulu

Ngwaketse 3:518 
Ngwana (Arabisés) 2:602

Ngwane 3:485

Ngwato 3:519

Ngwe. See: Bangwe

Nhominca. See: Nyominka

Nianga. See: Nyanga

Nika. See: Nyika

Nilamba. See: Nilyamba

Nilo-Hamitic 3:22

Nilotes. See: Nilotic

Nilotic (group) 1:581

Nilotic Kavirondo. See: Luo

Nilyamba 3:197

Ningo. See under Adangme

Nioniosse. See: Nyonyose

Niramba. See: Nilyamba

Njabi. See: Nzabi

Njamusi. See: Njemps

Njanja. See: Hera (and Njanja)

Njao. See: Ndau

Npawi. See: Nzabi

Njem. See: Maka-Njem

Njemps. See under Masai

Njevi. See: Nzabi

Njuwe. See under Tharaka

Nkole (Hima) 3:144

Nkom. See: Kom

Nkonya. See under Guang

Nkore. See: Nkole

Nkoya (Mbwela) 3:301

Nkundo 2:588 (incl. Ekonda).

See also Mongo

Nkundo-Mongo. See: Mongo

Nkutshu 2:625

Nkuvu 2:634

Nkwanta. See under Brong

Noho. See: Banaka

Nohwe. See under Rozwi

Non or Nono or Noon. See under Serer

Nri. See under Igbo

Nsakkara. See: Nzakara

Nsamba 2:589

Nsaw. See: Nso

Nsenga 3:301. See also: Senga

Nso. See under Tikar

Nsongo. Cp. Songo

Nsundi. See: Sundi

Nsungli. See under Tikar

Ntomba 2:614
Ntombe Njale. See under Ntomba

Ntshabeleng. See under Sotho (North)

Ntum. See under Fang

Nrumba. See: Ntomba

Ntumu. See: Ntum

Nuba 1:582

Nubian 1:565

Nuer 1:583

Nungu. See: Mada

Nupe 2:203 (incl. Kede)

Nweh. See: Bangwa

Nyabungu. See under Shi

Nyakwai. See under Labwor

Nyakyusa 3:197

Nyali 2:602

Nyam-Nyam. See: Zande

Nyamang. See under Nuba

Nyamwezi 3:199

Nyaneka 2:645

Nyang 2:405

Nyanga 2:607

Nyangbara. See under Bari

Nyanja 3:338

Nyankole. See: Nkole

Nyantruku. See under Nago

Nyaro. See under Nuba

Nyarwanda. See: Rwanda

Nyasa. See under Nyanja

Nyaturu. See: Rimi

Nyi 2:589

Nyiha-Safwa (group) 3:200

Nyika [Kenya] 3:104

Nyima or Nyimang. See: Nyamang

Nyiramba. See: Nilyamba

Nyixa. See: Nyiha-Safwa (group)

Nyominka. See under Serer

Nyongo. See under Bushongo

Nyonose. See under Moru-Madi

Nyonyose. See under Mossi

Nyoro 3:146

Nyungwe 3:350

Nzabi 2:504

Nzakara 2:500

Nzebi. See: Nzabi

Nzema or Nzima. 2:106

Okiek or Oggiek. See: Dorobo

Oli 2:614

Ometo. See under Sidama 
Omyene. See: Myene

Onga. See under Nkutshu

Oromo. See: Galla

Osu 2:107

Ouabou. See: Yoabu

Ouabouma. See: Boma [Zaïre]

Oubi. See: Ubi

Ouelleminden. See: Iullemmeden

Oulé. See: Wala

Oulliminden. See: Iullemmeden

Ouobe. See: Wobe

Ouolof. See: Wolof

Ouri. See: Oli

Ovaherero. See: Herero

Ovakuanyama. See: Kwanyama

Ovambo. See: Ambo

Owo. See under Yoruba

Oyo. See under Yoruba

Pääri. See under Anuak

Padhola. See: Adhola

Pagabete 2:589

Pahouin. See: Fang

Pakot. See: Pokot

Pama. See: Mpama

Pana 2:368

Pangwa. See under Hehe

Pangwe. See: Fang

Papel or Papéis. See under Mandyak

Parakuyo. See: Baraguyu

Pare. See: Asu

Pari. See: Beri

Peda 2:358

Pedi 3:479

Pende 2:614

Penin or Penyin. See: Banen

Pepel. See: Papel

Peul. See: Fulbe

Phalaborwa. See under Sotho (North)

Pika. See: Bolewa

Pilapila 2:359

Pimbwe 3:201

Podo. See: Bazantche

Pogoro 3:201

Poke 2:602

Poko. See: Bapuku

Pokomo 3:105

Pokot 3:105

Pomo 2:508

Pondo [South Africa]. See: Mpondo
Popo 2:359. Cp. also Gun

Popoi. See under Mangbetu

Poto 2:590

Pounou. See: Punu

Puku. See: Bapuku

Punu 2:504

Pygmy (Twide) 1:177, 2:543 (incl.

Baka, Efe). See also Babinga, Twa,

Mbuti, Bayele

Qafar. See: Afar

Qemant. See: Kemant

Qhalaxari. See: Kgalagadi

Quahoe. See: Kwahu

Quioco. See: Chokwe

Ragoli. See: Logoli

Rangi. See: Langi

Rega. See: Lega

Reguibat or Regibat 2:308

Remba. See: Lemba

Rembutu 3:324

Rendile 3:106

Reshiat. See: Geleba

Rgibat. See: Reguibat

Ribe. See under Nyika

Rimi 3:201

Roka. See under Sotho (North)

Rolong 3:519

Rom. See under Tikar

Ronga 3:350

Rooi Natie. See: Gai-Khan

Rotse. See: Lozi

Rozwi 3:324 [both ancient and existing peoples of that name. See also: Karanga]

Ruanda. See: Rwanda

Rubatab. See under Sudan Arab

Ruguru. See: Luguru

Rukuba 2:203

Rumbi. See under Mangbetu

Rundi 3:70

Ruri. See: Kwaya

Rwanda 3:71

Sa or Saa. See: Sakata

Saba. See: Hadjerai

Sabo. See under Kru

Sadama. See: Sidama

Sageju. See: Segeju 
Saho 1:633

Sahwi. See: Sefwi

Sakalava 3:372

Sakara. See: Nzakara

Sakata 2:615

Sala 3:301

Salampasu 2:625

Sama 2:646

Samba. See under Luba

Sambaa or Sambara. See: Shambala

Sambu. See under Mbundu(u)

Samburu 3:106

Samya. See under Luhya

San [Southern Africa]. See: Bushman

Sandawe 3:202

Sanga 2:634

Sangha-Sangha. See: Bomwali

Sangu [Tanzania]. See under Hehe

Sankani 2:590

Sanye 3:106

Sao. See under Kotoko

Sapo Sapo. See under Songye

Sara 2:493

Saraka. See: Tharaka

Sarakole. See: Soninke

Sarwa 3:519

Scinasci. See: Shinasha

Se Adangme. See: Shai

Sebei 3:147

Seereer. See: Serer

Sefwi 2:107

Segeju 3:202

Sehwi. See: Sefwi

Sekwele. See under Kung

Sena 3:351

Senga 3:302. See also: Nsenga

Sengele. See under Ntomba

Senufo 2:350

Serahuli. See: Soninke

Serer 2:314

Sese or Sesse 3:148

Shabe. See under Nago

Shaho. See: Saho

Shai. See under Adangme

Shaikiya or Shaigi or Shaiqiyah. See under Sudan Arab

Shalla. See under Kaleri

Shambala or Shambaa 3:202

Shangaan. See: Thonga

Shangalla or Shangul. See: Berta
Shankwe 3:325

Shawasha $3: 325$

Shawiya 1:505

Shaygiya. See: Shaikiya

Shekiri. See: Itsekiri

Sherbo. See also: Bulom

Shi 2:607. See also: Lele

Shila 2:634

Shilluk 1:585

Shilluk Luo. See: Jur

Shinasha 1:609

Shira. See under Punu

Shirazi. See: Swahili

Shomvi. See under Zaramo

Shona 3:325

Shukriya. See under Sudan Arab

Shukulumbwe. See: Ila

Sidama 1:609

Sidamo. See under Sidama

Siena. See Senufo

Sihanaka 3:373

Simbiti. See under Kuria

Simpi. See: Esimbi

Sinasha. See: Shinasha

Sirikwa (legendary) 3:106

Siwa 1:522

Sizaki. See under Kwaya

So. See: (1) Sao; (2) Sorat

Sobo 2:232 (incl. Isoko; Urhobo)

Soga 3:148

Soko [Central Africa]. See under Kele

Soli 3:302

Solongo 2:646

Somali 1:637

Somba (group) 2:359 (incl. Besorube, Betammaribe)

Somono. See under Bozo

Soney or Songai. See: Songhai

Songe. See Songye

Songhai 2:326. See also: Dyerma;

Dendi

Songhoi. See: Songhai

Songo 2:590 (Nsongo), 2:615, 2:625

(Basongo Meno) (incl. Atshuru)

Songola 2:608

Songye 2:625

Soninke 2:327. See also: Marka

Sonjo 3:203

Sonrai. See: Songhai

Soomaali. See: Somali 
Sooso. See: Sosso

Sorat (So). See under Karamojong

Sorkawa or Sorko [denoting Bozo]. See: Bozo

Sorongo. See: Solongo

Sorube. See: Biyobe

Soso [Angola]. See: Sosso

Soso [Guinée]. See: Susu

Sosso [Angola] 2:646

Sotho (North) 3:479. See also: Pedi

Sotho (Southern) 3:523

Sotho-Tswana 3:419. See also: Sotho; Tswana

Soundi. See: Sundi

Soussou. See: Susu

South Alada. See: Aizo

Subiya or Subia. See under Lozi

Subu 2:406

Sudan Arab 1:568

Sudie 2:331

Sugur. See: Sukur

Suk. See: Pokot

Suku 2:615

Sukuma 3:203

Sukur. See under Margi

Sumbwa. See under Nyamwezi

Sundi 2:508

Suri 1:586

Surma (group) 1:610

Susu 2:340

Suundi. See: Sundi

Swahili (Shirazi) 3:23 (incl. Unguja: Vumba). See also: Amu

Swazi 3:526

Sya. See: Tusia

Taabwa. See: Tabwa

Tabi. See: Ingassana

Tabwa 2:634

Tagwana or Tagbana or Tagouana. See under Senufo

Taimoro. See: Antemoro

Taita 3:107

Takruri 1:570

Talensi or Tallensi 2:107

Tambahooka or Tambahuaca. See: Antambahoaka

Tamberma. See: Betammaribe

Tambwa. See: Tabwa

Tañala 3:373
Tanda. See: Tenda

Tandroy. See: Antandroy

Taneka. See under Pilapila

Tanga. See: Batanga

Tangale 2:204

Tankamba. See: Yoabu

Tankarana. See: Antankarana

Tankay. See: Bezanozano

Tanosi. See: Antanosy

Taposa. See: Toposa

Tati Bushmen. See: Sarwa

Tatoga 3:205

Tau. See under Sotho (North)

Taveta 3:107

Tawana 3:519

Tawara 3:329

Tchambé. See: Bassari [Togo/ Ghana]

Tebu. See under Teda

Teda 2:494

Tefasy. See: Antefasi

Tegalawi. See under Nuba

Teita. See: Taita

Teke (group) 2:508

Tekruri. See: Takruri

Tem (Temba [Togo], Kotokoli) 2:379

Temba [Togo]. See: Tem

Temba [Zaïre]. See under Lunda

Tembu. See: Thembu

Temne 2:71

Temoro. See: Antemoro

Tenda 2:341

Tesaka. See: Antesaka

Teshi. See under $\mathrm{Ga}$

Teso 3:148

Tetela 2:626

Teuso. See under Karamojong

Teve 3:351

Tharaka 3:107

Thembu 3:496

Thonga 3:351 (Tonga, Tsonga, Shangaan), 3:481

Tiamus. See: Njemps

Tibu or Tibbu or Tibou. See under Teda

Tid. See under Surma. Cp. also Midob

Tiefo. See under Senufo

Tieli. See: Dieli 
Tikar (group) 2:406

Timne. See: Temne

Tindega. See: Hadzapi

Tiokossi. See: Tyokossi

Tiriki. See under Luhya

Tirma. See under Surma

Tiv 2:204

Tjimba. See under Herero

Tlhaping 3:496

Tlokwa 3:419

Tobu. See: Labwor

Tofoke. See: Poke

Togoyo. See under Feroge

Toka. See under: Tonga

Tokolor. See: Tukulor

Tokwa. See: Tlokwa

Toma. See: Loma

Tomba. See: Ntomba

Tonga 3:302 (General and Plateau

Tonga) (incl. Toka); 3:303 (Valley

Tonga or We); 3:329 (Toka,

Tonke): 3:339. See also: Thonga

Tonka. See under Tonga

Tooro. See: Toro

Topnaar. See under Nama

Topoke. See: Poke

Toposa 1:586

Topotha. See: Toposa

Toro [Uganda] 3:149

Torobo. See: Dorobo

Touareg. See: Tuareg

Toubouri. See: Tupuri

Toucouleurs. See: Tukulor

Toupouri. See: Tupuri

Toura. See: Tura

Toussian. See: Tusia

Tschokwe. See: Chokwe

Tsimihety 3:374 (For Tsimihety jointly with Sakalava, see Sakalava)

Tsogo 2:505

Tsokwe. See: Chokwe

Tsonga. See: Thonga

Tsotso [Angola]. See: Sosso

Tswa 3:353. See also: Twa

Tswana 3:519. See also:

Sotho-Tswana 3:419

Tuareg 1:505

Tuareg 2:327 (incl. Bella and Western Iullemmeden

Tuareg 2:331 (incl. Eastern
Iullemmeden). See also: Tuareg 1:505: 2:327

Tubu or Tubbu. See under Teda

Tuburi. See: Tupuri

Tufuki. See: Poke

Tukete. See: Kete

Tukolor or Tukulor. See under

Fulbe

Tulishi. See under Nuba

Tumba. See: Ntomba

Tumbatu 3:225

Tumbuka 3:339

Tupuki. See: Poke

Tupuri 2:407. See also under Mesa

Tura 2:350

Turkana 3:107

Turu. See: Rimi

Turuka 2:369

Tusia 2:369

Tussi. See: Tutsi

Tussian or Tusyan. See: Tusia

Tutsi (Hima) 3:72

Twa (Batwa, Batswa) (pygmies) 2:543; 2:646 (incl. Tyimba);

3:304. See also: Pygmy; Tswa

Twareg. See: Tuareg

Twi 2:108

Twide. See: Pygmy

Tyama. See: Ebrie

Tyap. See: Katab

Tyapi. See: Baga

Tyeli. See: Dieli

Tyimba. See under Twa

Tyo. See under Teke

Tyokossi 2:379

Ubi 2:350

Uduk or Udak. See under Koma

Ugep. See: Yakö

Ujang. See: Masongo

Ukuanyama. See: Kwanyama

Ule. See: Wiili

Uli. See: Oli

Umundri. See under Igbo

Unga 3:304

Unguja. See under Swahili

Ungwe (Hungwe). See under Manyika

Urhobo. See under Sobo

Uri. See: Oli 
Ushi. See: Aushi

Vai (Gallinas) 2:427

Vakpe. See: Kpe

Vakuanyama. See: Kwanyama

Valley Tonga. See under: Tonga

Valuimbe. See: Luimbi

Vandau or Vandzau. See: Ndau

Vasu. See: Asu

Vazimba 3:374

Vei. See: Vai

Venda 3:481

Veso. See: Vezo

Vezo 3:374

Vidunda 3:205

Vili 2:509 (incl. Cabinda)

Vira. See: Bira

Vouté. See: Vute

Vuma 3:149

Vumba [Tanzania]. See under Swahili

Vute 2:407

Waaba. See: Yoabu

Waata or Waboni. See: Boni

Wagenia. See: Enya

Wala 2:369

Walese. See: Balese

Wambu. See under Mbundu(u)

Wandala. See: Mandara

Wandji 2:505

Wanga. See: Hanga

Wangara. See under Mande

Wasu. See: Asu

Watutsi. See: Tutsi

We. See under: Tonga

Wè. See: Gere

Weppa-Wano. See under Benin

Western Iullemmeden (Western

Iwellemmeddän). See under Tuareg

Wiili. See under Dagari

Wiko. See: Luvale

Winjiwinji. See: Baseda

Wiya. See under Tikar

Wobe 2:350

Wodaabe. See under Fulbe

Wogo 2:332

Wolamo. See under Sidama

Wolof 2:315

Woro. See under Kresh

Wouri. See: Oli
Wouté. See: Vute

Woyo. See: Cabinda

Wule. See: Wiili. Cp. also: Wala

Wumbu. See: Humbu

Wuri. See: Oli

Wute. See: Vute

Wuum. See: Humbu

Xananwa. See: Malaboch

Xasonga or Xasonke. See: Khasonke

Xhosa 3:497

Xona. See: Shona

!Xũ. See under Kung

Xweda. See: Peda

Yaka 2:616

Yakö 2:232

Yakoma 2:590

Yalna 2:495

Yalunka. See: Dyalonke

Yambasa. See under Bafia

Yambo. See: Anuak

Yamonzombo. See: Monjombo

Yansi 2:617

Yao 3:339, 3:353

Yarse 2:369

Yaunde. See: Ewondo

Yebu. See: Ijebu

Yeke 2:635

Yela 2:590

Yembe 2:617

Yemsa. See: Janjero

Yidena. See: Buduma

Yoabu 2:360

Yombe 2:617

Yoruba 2:233. See also: Nago $2: 358$

Yowa. See: Pilapila

Yöyöose. See: Nyonyose

Yulu. See under Bongo-Baka

Zaberma. See: Dyerma

Zafimaniry. See under Tañala

Zaghawa 2:495

Zala. See under Sidama

Zamfarawa. See under Hausa

Zanaki 3:205

Zande 1:586. 2:602. See also: Bandia 2:596

Zaramo 3:205 
Zarma. See: Dyerma

Zegua or Zeguha. See: Zigua Zela 2:635

Zelmamu. See under Surma

Zerma. See: Dyerma

Zezuru 3:329

Ziba. See: Haya

Zigua or Zigula 3:206
Zimba [Mozambique] 3:353. See also: Vazimba 3:374

Zimba [Zaïre] 2:608

Zime. See: Maka-Njem

Zinza 3:206

Zompre. See: Kutep

Zulu 3:485

Zumper. See: Kutep 


\section{INDEX OF LANGUAGES}

The names of many languages have been subordinated to the names of larger groupings (indicated by "See under").

There are then 5 types of entry in this index:-

(1) a direct indication of the volume and page-number where entries on a language can be found: thus 2:352 refers to Volume 2, page 352

(2) "See references" from alternative spellings or names

(3) "See also references" when entries for a language can be found in more than one part of the Cumulative Bibliography

(4) "See under references" from the name of a language to the name of another language to which it has been subordinated; thus entries about Afutu will be found incorporated into those about Guang

(5) "Cp. references" when there is doubt whether two names really are alternatives or not.

Ababua. See: Bua

Abo. See: Bankon

Abonwa. See: Acoli

Abukeia. See: Logo-Avokaya

Abure 2:352

Acoli or Acholi or Acooli 3:155

Adangme or Adangbe 2:115

Adea. See: Hadiya

Adele 2:381

Adhola 3:155

Adiye. See: Hadiya

Adjer. See: Azer

Adouma or Aduma. See: Duma

Adyukru 2:352

Afar 1:633

Afrikaans 3:439

Afro-Asiatic. See under

Hamito-Semitic

Afusare. See: Jarawa

Afutu. See under Guang

Afuzare. See: Jarawa

Agau or Agaw (group) 1:619. See also

Bilin

Agni. See: Anyi

Ahanta. See under Nzima
Ajukru. See: Adyukru

Akan [Ghana] 2:116. See also Fante, Twi

Akoose. See: Kosi

Akwapem or Akuapim. See under Twi

Akye 2:352

Alaba. See under Sidamo

Aladyan or Aladian or Alagian 2:352

Alago. See: Arago

Alagwa. See under Iraqw

Alagya. See: Aladyan

Alawa. See: Alagwa

Alladian. See: Aladyan

Alur 2:604, 3:155

Amarinya. See: Amharic

Ambo (group) 3:509. See also

Kwanyama; Ndonga

Amharic 1:619

Amu. See under Swahili

Angas 2:206

Angba 2:604

Animere. See under Tem

Anjouanais. See: Nzwani

Ankober. See: Argobba

Ano. See: Anufo 
Anuak 1:590

Anufo 2.381

Anupe. See: Nupe

Anyi 2:352

Anyuak or Anywa. See: Anuak

Aowin. See: Brisa

Apagibeti or Apakabeti. See: Pagabete

Arabic 1:246. See also Sudan Arabic

Arago. See under Idoma

Aramanik. See under Dorobo

Arbore. See under Konso-Geleba

Argobba 1:622

Ashanti or Asante. See under Twi

Asu (Chasu, Pare) 3:213

Aten. See: Ganawuri

Ateso. See: Teso

Athu. See: Asu

Atshokwe. See: Chokwe

Atye. See: Akye

Auni. See under Bushman

Aushi. See under Bemba

Avatime 2:381

Avikam 2:352

Avokaya. See: Logo-Avokaya

Awiya. See under Agau

Awowin. See: Brisa

Awutu. See under Guang

Azande. See: Zande

Azer 2:328

Bachama 2:206

Badawi. See under Kanuri

Bade (group) 2:206

Badyaranke or Badian. See under

Tenda

Bafia 2:412

BafueNuma. See under Nyali

Bagam 2:412

Bagirmi 2:496

Bai [Chad]. See: Mbai

Bai [Sudan]. See: Bari

Baiso 1:623

Baja. See: Baya

Bakoko 2:412

Bakossi. See: Kosi

Bakweli or Bakwiri. See: Kpe

Balanta 2:436

Balese 2:604

Bali [Cameroun] 2:412

Bamana. See: Bambara
Bambala [Ethiopia]. See under Konso-Geleba

Bambara 2:328. See also: Mande

Bamileke 2:412

Bamoun. See: Bamum

Bamum 2:413

Bamun. See: Bamum

Banda [République Centrafricaine] 2:500

Bandem. See under Bamileke

Bandi 2:429

Banen. See: Tunen

Bangangte. See under Bamileke

Bangi (Bobangi) 2:590. See also: Yansi

Bangoua. See: Bangwa

Bangubangu 2:609

Bangwa. See under Bamileke

Bankon 2:413 [Out of order]

Banmana. See: Bambara

Banoho. See under Batanga

Bantu 1:246

Baoulé. See: Baule

Barea. See: Baria

Bari 1:590

Baria 1:633

Barma. See: Bagirmi

Basa [Cameroun] 2:413 (incl. Mbang)

Basa [Liberia]. See: Bassa [Liberia]

Basaa. See: Basa [Cameroun]

Basari [Côte d'Ivoire]. See under Tenda

Basari [Togo]. See: Bassari [Togo]

Basekwe 2:653

Bashama. See: Bachama

Basic Bantu. See: Fanagalo

Bassa [Cameroun]. See: Basa

Bassa [Liberia] 2:429

Bassari [Côte d'Ivoire]. See: Basari [Côte d'Ivoire]

Bassari [Togo] 2:381

Batanga 2:413 (incl. Banoho)

Batswa or Batwa. See: Twa

Baula. See: Brame

Baule or Bawule 2:352

Baya 2:500

Bayso. See: Baiso

Beafada. See: Biafada

Bebejato. See under Nkom 
Bedawiye or Bedauye 1:570

Bedde or Bede. See: Bade

Bedik. See under Tenda

Bedja. See: Bedawiye

Beir. See: Murle

Beja. See: Bedawiye

Bemba 3:305

Bembe [Congo Republic] 2:510

Bembe [Zaïre] 2:609

Bena. See under Hehe

Bena Lulua. See: Lulua

Benga 2:654

Benue-Congo (group) 2:413

Beo. See under Angba

Berber 1:472. 1:493, 1:496, 1:515, 1:520. See also: Kabyle

Berta or Bertha 1:623

Berti 1:570

Besarin. See: Bisharin

Bete 2:352

Biafada 2:436

Bicharin. See: Bisharin

Bideyat 2:496

Bikom. See: Nkom

Bila. See: Bira

Bilin or Bilen 1:633

Bimoba. See: Moba

Bindji. See: Mbagani

Binga [Sudan]. See under Kresh

Bini (Edo) 2:243

Binji. See: Mbagani

Bira 2:604

Birged. See under Nubian

Biri. See: Birri

Birom 2:206

Birri. See under Ndogo

Bisa [Burkina Faso] 2:369. See also: Busa

Bisa [Zambia]. See under Lala

Bisharin or Bisciarino. See under Bedawiye

Ble 2:370

Bo [Bunkina Faso] 2:370

Bo [Cameroun]. See: Bankon

Boa. See: Bua

Bobangi. See: Bangi

Bobe. See: Bubi

Bobo 2:370

Bodo 2:635

Boka. See: Bo [Burkina Faso]
Boko [Nigeria]. See under Busa

Bolanci or Bole or Bolewa. See under Bade

Bolia. See under Ntomba

Bondei 3:214

Bongo-Baka 1:590

Boni [Somalia] 1:644

Bor. See under Dinka

Boran or Borana. See under Galla (Oromo)

Bornu. See: Kanuri

Bouamou. See: Bwamu

Boulou. See: Bulu

Bowiri 2:381

Bozo 2:328

Brame. See under Mandyak

Brisa or Brissa or Brossa or Brussa. See under Anufo

Bua 2:604

Bubi 2:655

Budja 2:591

Budu. See: Bodo

Buduma 2:496

Budya 2:626

Builande. See under Songye

Bujeba [Guinea Ecuatorial] 2:654

Bulom 2:74

Bulu 2:413

Bulunge. See: Burungi

Bum. See: Mbum

Bundo. See: Mbundu(u)

Bura-Mabang. See: Maba

Buressya. See: Brisa

Burji. See: Bambala

Burungi. See under Iraqw

Busa 2:206

Bushman 3:439 (Sarwa). See also Khoisan

Bushongo or Bushoong 2:626 (incl. Lele)

Buti. See: Vute

Buwe. See: Mbugwe

Bviri. See: Birri

Bwaka. See: Ngbaka

Bwamu. See under Bobo

Bwyo. See under Mbugu

Caffa or Caffino. See: Kafa

Caga. See. Chaga

Cakosi. See: Anufo 
Cama. See: Ebrie

Cambata. See: Kambatta

Cangin 2:317

Caxa. See: Chaha

Cebaara. See: Tyembara

Cewa 3:340. See also Nyanja

Chadic or Chado-Hamitic 2:496

Chaga or Chagga 3:214

Chaha. See under Gurage

Chakosi. See: Anufo

Chaouia. See: Shawiya

Chasu. See: Asu

Chewa. See: Cewa

Chibuk. See: Cihak

Chokosi. See: Anufo

Chokwe 2:647

Chopi 3:354

Chwabo or Chuabo 3:354

Cibak. See under Margi

Ciga. See: Kiga

Cioko or Ciokwe. See: Chokwe

Classical Ethiopic. See: Ge'ez

Cokwe. See: Chokwe

Comorian. See also: Nzwani

Congo. See: Kongo

Congo Swahili [i.e. Ngwana]. See:

Ngwana

Copi. See: Chopi

Creole 1:253. See also Krio

Cuabo. See: Chwabo

Cuanhama. See: Kwanyama

Cunama. See: Kunama

Daba 2:414

Dabida. See under Taita

Dabosa. See: Toposa

Dagari or Dagara 2:116

Dagomba or Dagbamba or Dagbane 2:116

Dahomey Yoruba. See: Nago

Dair. See under Nubian

Dama [Liberia]. See under Vai

Dan 2:429

Danakil or Dancalo. See: Afar

Dangaleat 2:496

Dangme. See: Adangme

Darasa. See under Sidamo

Dasa. See: Daza

Datoga. See: Tatoga

Dawida. See: Dabida
Daza. See under Teda

Dciriku. See: Diriku

Delen. See under Nubian

Denka or Denkawi. See: Dinka

Dera. See: Kanakuru

Derasa. See: Darasa

Dho Aluur. See: Alur

Dho-Luo. See: Luo

Dhopadhola. See: Adhola

Dian. See: Dyan

Didinga 1:590

Digo. See under Nyika

Dilling. See: Delen

Ding. See: Dzing

Dinka 1:590

Diola. See: Dyola

Diriku 2:647

Djao. See: Yao

Djerma. See: Dyerma

Djok. See: Chokwe

Djoukoun. See: Jukun

Dogon 2:329

Doko [Zaïre] 2:591

Dompago. See: Logba

Don. See under Samo

Dongolawi. See under Nubian

Dorobo (Okiek) 3:114

Douala. See: Duala

Douma. See: Duma

Dschagga. See: Chaga

Duala 2:414

Duka. See under Kambari

Duma [Gabon] 2:505

Dwala. See: Duala

Dyan or Dya 2:370

Dyerma 2:332. See also Songhai

Dyola 2:317

Dzalamo. See: Zaramo

Dzing 2:618

East Sidama. See: Sidamo

Ebira. See: Igbira

Ebrie 2:352

Ebudu. See: Bodo

Echwabo. See: Chwabo

Ecisubia. See: Subiya

Edo. See: Bini

Effutu. See under Guang

Egbiri. See: Igbira

Egede. See: Igede 
Eghap. See: Bagam

Ehoué. See: Ewe

Ekihaya. See: Haya

Ekipangwa

Ekoi 2:244

Ekonda. See: Konda

Eloyi. See under Idoma

Endo. See under Pokot

Engassana. See: Ingassana

English 1:144

Enyong. See under Ekoi

Erbore. See: Arbore

Erokh. See: Iraqw

Esako. See under Bini

Eshwabo or Etcwabo. See: Chwabo

Ethiopic, Classical. See: Ge'ez

Etsako. See: Esako

Etulo 2:206

Etung. See: Nkom

Evhe. See: Ewe

Ewe or Evhe 2:381

Ewodi. See: Wori

Ewondo 2:414

Fada. See: Biafada

Fali 2:415

Fanagalo 3:440

Fang 2:505, 2:654

Fante 2: 116. See also Twi

Fazoglo. See under Berta

Fer. See: Kara

Feroge 1:591

Fiome. See under Iraqw

Fiote. See: Vili

Fipa 3:214

Fizere. See: Jarawa

For. See: Fur

French 1:147

Fuk 2:415

Fula (Ful or Fulani or Fulfulde) 2:42, $2: 206 ; 2: 415$

Fumu. See under Teke

Fur 1:570

Fute. See: Vute

Ga 2:117

Ga'anda. See under Tera

Gabin. See: Ga'anda

Gafat 1:623

Gaingbe. See: Mina
Galke. See under Mbum

Galla (Oromo) 1:623

Gamila. See under Berta

Ganawuri 2:207

Ganda [Nigeria]. See: Ga'anda

Ganda [Uganda] 3:156

Gang. See: Acoli

Ganguela. See: Ngangela

Gbagyi. See: Gbari

Gbanda. See: Avikam

Gbandi. See: Bandi

Gbari 2:207

Gbasa. See: Bassa [Liberia]

Gbaya [Central Africa Republic]. See:

Baya

Gbaya [Sudan]. See: Kresh

Gbedegi. See under Nkom

Gbeya. See: Baya

Gboare. See: Bachama

Gciriku. See: Diriku

Ge. See under Ewe

Ge Sinan. See: Harari

Gede'uffa. See: Darasa

Geechee. See: Gullah

$\mathrm{Ge}^{6}$ ez 1:624

Gekoyo. See: Kikuyu

Gen. See: Ge

Gengbe. See: Mina

Gerzé 2:342 See also Kpelle

Geshu. See: Gisu

Ghez. See: Ge'ez

Giangerò. See: Janjero

Gidole. See under Konso-Geleba

Giha. See: Ha

Gikuyu. See: Kikuyu

Gimira-Maji (group) 1:625

Gio. See: Dan

Giryama. See under Nyika

Gishu. See: Gisu

Gisiga 2:415

Gisu 3:157

GiTonga. See under Tonga

Glavda. See under Mandara

Goali. See: Gbari

Gogo (group) 3:214

Gola [Liberia] 2:429

Golo 1:591

Gonja [Ghana]. See: Guang

Goroa. See under Iraqw

Goula. See: Gula 
Gouraghe. See: Gurage

Gourma or Gourmantche. See: Gurma

Gourounsi. See: Grusi

Gowaze. See under Konso-Geleba

Grand Comorien. See: Ngazidja

Grebo. See under Kru

Grikwa or Griekwa or Griqua. See under Hottentot

Grusi 2:370

Guang 2:117 (incl. Nkunya)

Guerzé. See: Gerzé

Guèze. See: Ge'ez

Guingbe. See: Mina

Guissiga. See: Gisiga

Gula [Chad] 2:496

Gullah. See under Creole

Gulmance or Gulmancema. See: Gurma

Gumuz. See under Koma

Gur 2:43

Gurage 1:625

Gurenne 2:118

Gurma or Gurmantche 2:370

Gurunsi. See: Grusi

Gusii 3:114

Gwali. See: Gbari

Gwamba or Gwapa. See under Thonga

Gwari. See: Gbari

Gweabo. See under Kru

Ha 3:215

Hadendowa. See under Bedawiye

Hadiya 1:626

Hamito-Semitic (Afro-Asiatic) 1:254

Haoussa. See: Hausa

Harari 1:626

Haruro. See under Ometo

Hasania. See: Hassanya

Hassanya 2:309

Hausa 2:207

Haya 3:215

Hehe (group) 3:215

Heiban. See under Koabib-Tagoi

Hemba 2:635

Henga. See under Tumbuka

Herero 3:510

Heso. See: So

Hide 2:415
Higi 2:415

Hill Nubian. See: Nubian ('Hill' Nubian)

Hitkalanci. See: Bolanci

Holoholo 2:635

Hottentot 3:498. See also Khoisan; Korana; Nama

Hunde 2:609

Hungu 2:647

Ihembe. See: Bembe [Zä̈re]

Ibibio-Efik 2:244

Ibo. See: Igbo

Icisubra. See: Subiya

Idoma 2:213

Idzing. See: Dzing

Igala or Igara or Igarra 2:213

Igbira 2:213

Igbo $2: 244$

Igede. See under Idoma

Igiha. See: $\mathrm{Ha}$

Ijo or Ijaw 2:246

Ik. See under Karamojong

Ika. See under Igbo

Ikiha. See: $\mathrm{Ha}$

Ikirundi. See: Rundi

Ila 3:306

Ilamba. See: Nilyamba

Indri. See under Feroge

Ingassana or Ingessana 1:591

Iraku. See: Iraqw

Iramba. See: Nilyamba

Irangi. See: Langi

Iraqw (group) 3:215

Irbore. See: Arbore

Irob. See under Saho

Isala 2:118

Isilolo. See: Fanagalo

Isiswati. See: Swazi

Isu or Isubu. See: Subu

Iyanzi. See: Yansi

Izere. See: Jarawa

Izi 2:246

Izon. See: Ijo

Jang. See: Dinka

Janjero 1:626

Jao. See: Yao

Jarawa 2:213

Jegu 2:496 
Jibu. See under Jukun

Jieng. See: Dinka

Jita. See: Kwaya

Jola. See: Dyola

Jopadhola. See: Adhola

Jukun or Jukon 2:213

Juman 2:496

Jur. See: Lwo

Kabin. See: Ga'anda

Kabre or Kabiye or Kabye 2:383

Kabyle 1:509

Kafa or Kaffa or Kafficho 1:627

Kafir. See: Xhosa

Kaguru. See under Gogo

Kahonde. See: Kaonde

Kakongo. See under Vili

Kakwa. See under Bari

Kalenjin. See under Nilo-Hamitic

Kamanga. See under Tumbuka

Kamba [Kenya] 3:114

Kambari 2:213

Kambatta. See under Sidamo

Kami 3:216

Kanakuru. See under Tangale

Kanda. See: Ga'anda

Kanembu 2:496

Kanuri 2:213

Kanyoka 2:626

Kaonde 3:306

Kapsiki. See: Higi

Kara [Sudan] 1:571

Karamojong 3:157

Karanga 3:329

Karekare. See under Bade

Karimojong. See: Karamojong

Kasele 2:383

Kasem or Kasena. See under Grusi

Kasena. See: Kasem

Kebu 2:383

Kegem or Kegueme. See under Serer

Kele [Gabon] 2:505

Kele [Zaïre] (Lokele) 2:604

Kemant. See under Agau

Kenuz or Kunuz. See under Nubian

Kerewe or Kerebe 3:216

Kete 2:627

Khamir. See under Agau

Khoekhoe or Khoekhoegowab

[formerly called Nama]. See: Nama
Khoi. See: Hottentot

Khoi-Khoi. See: Nama

Khoisan (Bushman and Hottentot) 3:440. See also separately:

Bushman; Hottentot

Khomani. See under Bushman

Khung. See: Kung

Kiama. See: Ebrie

Kiga. See under Nkore

Kiha. See: $\mathrm{Ha}$

Kikuyu 3:114

Kimant. See: Kemant

Kimbu. See under Nyamwezi

Kinga. See under Nyakyusa

Kinyarwanda. See: Rwanda

Kioko. See: Chokwe

Kisi 2:342

Kisii. See: Gusii

Kitchen Kafir. See: Fanagalo

Kituba. See: Tuba

Koalib-Tagoi (group) 1:591

Kofyar 2:214

Koko. See: Bakoko

Kolango. See: Kulango

Kololo. See: Lozi

Kom. See: Nkom

Koma [Ethiopia] 1:627

Kombe [Guinea Ecuatorial] 2:654

Komo. See: Kumu

Konda 2:618

Konde. See: Nyakyusa

Kongo 2:460, 2:619. See also Tuba

Konjara. See: Fur

Konjo 3:157

Konkomba 2:118

Konso-Geleba (group) 1:627

Kony. See under Nandi

Konzo. See: Konjo

Koongo. See: Kongo

Korana 3:498

Koranko 2:74

Kosi 2:415

Kotoko 2:415

Koulango. See: Kulango

Kouranko. See: Koranko

Kouroumba. See: Kurumba

Kpe 2:416

Kpelle 2:429

Kposo 2:383

Krebo. See: Grebo 
Kresh 1:591

Krim. See under Bulom

Krio 2:74

Krobo. See under Adangme

Krou. See: Kru

Kru 2:429

Kuangali. See: Kwangali

Kuba. See: Bushongo

Kukuruku. See under Bini

Kulã-bo. See: Kulango

Kulango 2:352

Kulia. See: Kuria

Kumbi. See under Nyaneka

Kumu 2:605

Kunama 1:633

Kundu 2:416

Kung or !Kung 3:510

Kunuz. See: Kenuz

Kunya. See. Nkunya

Kuranko. See: Koranko

Kuria 3:216

Kurumba 2:370

Kusasi or Kusal 2:118

Kwa 2:44

Kwafi. See under Masai

Kwambi. See under Ndonga

Kwangali 3:510

Kwanyama 3:510

Kwara or Quara. See under Agau

Kwaya 3:216

Kyama. See: Ebrie

Kyenga. See under Busa

Laadi. See: Lari

Laamang 2:214

Ladi. See: Lari

Laka [Chad]. See under Sara

Lala [Zambia] 3:306 (including Bisa;

Swaka), 3:489

Lali. See under Teke

Lamang. See: Laamang

Lamba [Togo]. See under Tem

Lamba [Zambia] 3:306. See also Lala for Lala-Lamba

Lambya. See under Nyiha-Safwa

Lamnso. See: Nsaw

Lamu. See: Amu

Landogo. See: Loko

Langi [Tanzania] 3:216

Lango 3:157
Lari 2:510

Latuka. See: Lotuko

Lefana $2: 383$

Lega 2:609

Legba. See: Logba

Lekpokpam. See: Konkomba

L'ele [Burkina Faso]. See: Lyele

Lele [Chad]. See under Nancere

Lele [Zaïre]. See under Bushongo

Lelemi. See: Lefana

Lendu 2:605

Lenge. See: Chopi

Lengi. See: Lenje

Lengola. See under Bira

Lengora. See: Lengola

Lenje 3:306

Lese. See: Balese

Lete [Ghana]. See under Guang

Lilima. See under Shona

Limba [Sierra Leone] 2:74

Limi. See: Rimi

Llugule. See: Logoli

Lobangi. See: Bangi

Lobedu 3:482

Lobi 2:370

Logba 2:383

Logbara. See: Lugbara

Logo-Avokaya 2:605

Logoli. See under Gusii

Logone. See under Kotoko

Loikop. See under Masai

Lokele. See: Kele

Loko. See under Mende

Lokonda. See: Konda

Lolo. See: Lomwe

Loma 2:430

Lomwe 3:354

Londo. See: Lundu

Lonkundo. See: Nkundo

Lotuko or Lotuxo 1:591

Lovale. See: Luvale

Lovedu. See: Lobedu

Lozi 3:306

Luba 2:627: 2:635 (incl. Samba)

Luba-Hemba. See: Hemba

Luba Lulua. See: Luba

Lubedu. See: Lobedu

Lugbara 3:157

Lugoli. See: Logoli

Luguru 3:216 
Luhya 3:115

Lukpa. See: Logba

Lulua 2:628

Lunda 2:636, 3:307

Lundu 2:416

Luo 3:115

Lur. See: Alur

Luvale 3:307

Luyana. See: Lozi

Luyia. See: Luhya

Lwana. See under Nyamwezi

Lwena. See: Luvalè

Lwo 1:591

Lyele 2:370

Ma'a. See: Mbugu

Maasai. See: Masai

Maba [Chad] 2:497

Mabale. See under Ngala [Zaïre]

Mabar 2:497

Mabendi. See under Mamvu

Macame. See: Machame

Machame. See under Chaga

Macua. See: Makua

Madi. See: Logbara

Maghavul. See: Sura

Maha 2:214

Mahas. See under Nubian

Majang. See: Masongo

Makere. See under Mangbetu

Makhuwa or Makoa. See: Makua

Makonde 3:354

Makua 3:354

Malinke (Mandingo) 2:342. See also Mande

Mambai 2:416

Mambila 2:416

Mambwe 3:307

Mamvu 2:605

Mandara 2:416

Mande (Mandingo) 2:44. See also

Bambara; Malinke

Manding. See: Mande

Mandingo. See: Malinke; Mande

Mandinka. See: Malinke

Mandjaque. See: Mandyak

Mandyak 2:436

Manga. See under Kanuri

Mang'anja. See under Nyanja

Mangaya. See under Feroge
Mangbetu 2:605

Maninka. See: Malinke

Manjaku. See: Mandyak

Mankanya. See under Mandyak

Mano 2:430

Manya 2:430

Manyika 3:330

Mapan or Mapun. See: Sura

Maragoli. See: Logoli

Margi 2:214

Masa 2:416

Masaba. See: Gisu

Masai (Kwafi, Loikop or Oigob) 3:216

Masana. See: Masa

Mashami. See: Machame

Mashi. See: Shi

Masingo. See: Masongo

Masongo [Ethiopia] 1:627

Massa. See: Masa

Matengo 3:216

Matumbi 3:217

Maviha 3:355

Mavul. See: Sura

Mawer. See: Mabar

Mayduub. See: Midob

Mayogo 2:605

Mba 2:605

Mbacca. See: Ngbaka

Mbagani 2:627

Mbai. See under Sara

Mbaka. See: Ngbaka

Mbali [Angola] 2:647

Mbang. See under Basa [Cameroun]

Mbangala. See under Mbundu(ki)

Mbanza [Zaïre]. See under Ngala [Zaïre]

$M$ be [Cameroun]. See under Nyang

Mbede 2:505

Mbembe [Nigeria] 2:246

Mbesa 2:605

Mboshi or Mbochi or Mbosi 2:510

Mboum. See: Mbum

Mbugu [Tanzania] 3:217

Mbugwe 3:217

Mbui [Angola]. See under

Mbundu(ki)

Mbulunge. See: Burungi

Mbum 2:416

Mbunda 2:647

Mbundu(ki) 2:647 
Mbundu(u) 2:648

Mbuza. See: Budja

Me'en. See: Mekan

Medje. See under Mangbetu

Meidob. See: Midob

Meje. See: Medje

Mekan or Mek'en 1:627

Mende 2:75

Meqan. See: Mekan

Meroitic 1:571

Merule. See: Murle

Mesengo. See: Masongo

Mfang. See: Fang

Mfinu 2:620

Midob. See under Nubian

Milembwe 2:629

Mimi 2:497

Mina. See under Ewe

Mine Kafir. See: Fanagalo

Minianka or Minyanka. See under

Senufo

Mituku 2:605

Moab. See: Moba

Moba [Ghana/Togo] 2:383

Moca. See: Mocha

Mocha. See under Kafa

Mochi or Moci. See: Moshi

[Tanzania]

Mogogodo 3:115

Molé. See: Mossi

Momfu or Momvu. See: Mamvu

Mondari. See: Mundari

Mondunga. See under Ngala [Zaïre]

Mongo ('Lolo') 2:591. See also

Nkundo

Moore or Moré. See: Mossi

Moru-Madi (group) 1:591

Moshi or Mosi [Tanzania]. See under Chaga

Mosiro. See under Dorobo

Mossi 2:370

Moubi. See: Mubi

Mpangwe. See: Fang

Mpondo 3:499

Mubi 2:497 [out of order]

Mukogodo. See: Mogogodo

Mum. See: Bamum

Mundari. See under Bari

Mungaka. Cp. Bali

Munjuk. See: Musgu
Mupun. See: Sura

Murle 1:592

Musei. See under Masa

Musgu 2:417

Musseronga. See: Solongo

Mussey. See: Musei

Musuk. See: Musgu

Muzey. See: Musei

Mwaghavul. See: Sura

Mwela. See: Mwera

Mwera 3:217

Mwohovul. See: Sura

Myene 2:506

Nafana or Nafara 2:118

Nago (Dahomey Yoruba) 2:361

Naho. See: Nao

Nama 3:511. See also Khoisan; Hottentot

Nama-Hottentot. See: Nama

Namakere-ti. See: Makere

Namakwa. See: Nama

Namejeti. See: Medje

Namwezi. See: Nyamwezi

Nancere 2:497

Nande 2:609

Nandi 3:115

Nankani. See: Gurenne

Nantchoa. See under Nancere

Nao or Nayi or Nayo. See under Gimira-Maji

Ndali. See under Nyiha-Safwa

Ndau 3:355

Ndebele 3:330, 3:482

Ndjevi. See: Nzabi

Ndogo 1:592

Ndonga 3:512

Ndorobo. See: Dorobo

Ndumu. See under Mbede

Ndunga or Ndunga-le. See: Mondunga

Ndzawu. See: Ndau

Ndzundra. See under Ndebele

Nembe. See under Ijo

Neyo 2:352

Ngala [Sudan] 1:592

Ngala [Zaïre] 2:592

Ngangela 2:648

Ngara. See: Ngala [Sudan]

Ngas. See: Angas 
Ngazidja 3:381

Ngbaka 2:593

Ngbandi 2:593

Ngelima. See under Angba

Ngemba. See under Nkom

Ngizim. See under Bade

Ngombe 2:593

Ngonde. See: Nyakyusa

Ngoni [Malawi] 3:340

Ngoni [Tanzania] (Sutu) 3:217

Ngumba 2:417

Nguni 3:440

Ngwa. See: Bangwa

Ngwana 2:566

Ngwe. See: Bangwa

Niam-Niam. See: Zande

Nibula. See: Nuna

Nika. See: Nyika

Nilo-Hamitic (group) 3:40

Nilotic (group) 1:592

Nilyamba 3:2 17

Nimbi. See: Nembe

Niramba. See: Nilyamba

Niwoli. See: Neyo

Njao. See: Ndau

Njebi. See: Nzabi

Nkengo. See under Mongo

Nkole. See: Nkore

Nkom 2:417

Nkonya. See: Nkunya

Nkore [Uganda] 3:157

Nkosi. See: Kosi

Nkundo 2:593. See also Mongo

Nkundo-Mongo. See: Mongo

Nkunya. See under Guang

Nkutshu 2:629

Noho or Noko. See: Banoho

Nsaw. See under Nkom

Nsenga 3:307

Nsibidi (secret language and script) $2: 246$

Nso'. See: Nsaw

Ntomba 2:620

Ntumba. See: Ntomba

Nubian ('Hill' Nubian) 1:592 (including Dair; Delen or Dilling); 1:571 (including Dongolawi;

Birged; Kenuz or Kunuz; Mahas;

Midob)

Nuer 1:592
Nugzum. See: Ngizim

Nuna [Burkina Faso]. See under Grusi

Nupe 2:214

Nuruma. See: Nuna

Nyakyusa 3:217

Nyali 2:605

Nyam-Nyam. See: Zande

Nyamwezi 3:217

Nyaneka 2:648

Nyang 2:417

Nyanga 2:609

Nyangbara. See under Bari

Nyangbo. See under Avatime

Nyanja 3:340. See also Cewa

Nyankole or Nyankore. See: Nkore

Nyarafolo. See under Senufo

Nyarwanda. See: Rwanda

Nyasa. See under Nyanja

Nyaturu. See: Rimi

Nyiha-Safwa 3:218

Nyika 3:116

Nyole. See under Luhya

Nyoro 3:158

Nyungwe 3:355

Nzabi 2:506

Nzebi. See: Nzabi

Nzima or Nzema 2:118

Nzwani 3:381

Obamba [Gabon]. See: Mbede

Obere Okaimie ('spirit' language and script) $2: 246$

Ociherero. See: Herero

Odzukru. See: Adyukru

Ogoni 2:246

Ohuhu. See under Igbo

Oigob. See: Loikop

Okiek. See: Dorobo

Okonyong. See under Ekoi

Oli. See: Wori

Olombo. See under Kele [Zaïre]

Olunyoro. See: Nyoro

Omati. See: Ometo

Ombamba. See: Mbede

Ombo 2:609

Ometo (group) 1:627

Oromo. See: Galla

Oron. See under Ibibio-Efik

Orunyoro. See: Nyoro 
Orusyan. See: Syan

Otetela. See: Tetela

Otjiherero or Otyiherero. See: Herero

Ouolof. See: Wolof

Ouri. See: Wori

Ovambo. See: Ambo

Oxoriok. See under Lotuko

Padhola. See: Adhola

Paduko. See under Mandara

Pagabete 2:594

Pagibeti. See: Pagabete

Pahouin. See: Fang

Pajade. See: Badyaranke

Pakabete. See: Pagabete

Pamue. See: Fang

Pana 2:371

Pangwa. See under Hehe

Pangwe. See: Fang

Papel or Papéis. See under Mandyak

Pare. See: Asu

Pedi. See: Sotho (North)

Pende 2:620

Pepel. See: Papel

Peul. See: Fula

Pfokomo. See: Pokomo

Phuthi 3:524

Pidgin. See under Creole

Pindi. See: Pende

Pla. See under Kru

Pogoro 3:218

Pokomo 3:116

Pokot 3:116

Pondo [South Africa]. See: Mpondo

Popo. See under Ewe

Pounou. See: Punu

Punu 2:506

Pygmy 1: 255. See also in vol. 2: Twa p. 567

Qafar. See: Afar

Qemant. See: Kemant

Quara. See: Kwara

Quioco. See: Chokwe

Ragoli. See: Logobi

Rambia. See: Lambya

Randile. See: Rendile

Rega. See: Lega

Rek. See under Dinka
Rekpa. See: Bafia

Rendile 3:116

Reshe 2:215

Rikpak. See: Bafia

Rimi 3:218

Ron 2:215

Ronga 3:355

Ruanda. See: Rwanda

Ruguru. See: Luguru

Rundi 3:75

Rwanda 3:75

Saa. See. Sakata

Sagatu. See: Berti

Saho 1:634

Sakata 2:620

Samatengo. See: Matengo

Samba [Zaïre]. See under Luba

Sambara. See: Shambala

Samo or San [Burkina Faso] 2:371

San [Southern Africa]. See: Bushman

Sandawe 3:218

Sanga 2:636

Sango 2:501

Sanhaja. See: Zenaga

Santrokofi 2:383

Sanye 3:116

Sara 2:497

Saraka. See: Tharaka

Sarakole. See: Soninke

Saramo. See: Zaramo

Sarwa. See under Bushman

Sayanci 2:215

Sebei 3:158

Sechuana. See: Tswana

Sedere. See: Adele

Seereer. See: Serer

Sele. See: Santrokofi

Semito Hamitic. See: Hamito-Semitic

Sena 3:356

Senadi. See under Senufo

Senga. See: Nsenga

Senufo 2:352

Sepedi. See: Sotho (North)

Seraculeh. See: Soninke

Serer 2:317

Sesuto. See: Sotho (Southern)

Sha. See under Ron

Shaho. See: Saho

Shambala 3:218 
Shanga. See under Busa

Shashi. See under Kwaya

Shawiya 1:509

Sherbro. See: Bulom

Shi 2:609

Shilluk 1:593

Shindzuani. See: Nzwani

Shingazidja. See: Ngazidja

Shinzwani. See: Nzwani

Shona 3:330. See also Zezuru and other dialects

Shukulumbwe. See: Ila

Sidamo (group) 1:627

Siha. See under Chaga

Sisala. See: Isala

Sisuthu. See: Sotho (Southern)

Siswati. See: Swazi

Siwi 1:523

Siya. See: Avatime

So [Cameroun]. See under Kotoko

So [Zaïre]. See under Kele [Zaïre]

Sobo (Urhobo) 2:246

Soga 3:158

Soko. See: So

Soli 3:307

Solongo 2:648

Somali 1:639

Soney. See: Songhai

Songe. See: Songye

Songhai or Songoi 2:329. See also: Dyerma

Songye 2:629

Soninke 2:329

Sonrhay. See: Songhai

Soomali. See: Somali

Sooninke. See: Soninke

Sorongo. See: Solongo

Soso. See: Susu

Sotho (North) 3:482

Sotho (Southern) 3:525

Sotho-Tswana 3:441

Souto. See: Sotho (Southern)

Subiya. See under Lozi

Subu 2:417

Sudan Arabic 1:572

Suk. See: Pokot

Sukulumbwe. See: Ila

Sukuma 3:219

Sumbwa. See under Nyamwezi

Sungor. See under Tama
Sura. See under Angas

Susu 2:343

Sutu. See: Ngoni

Șuura. See: Sura

Suwu. See: Subu

Swahili 3:40. See also Ngazidja; Ngwana; Nzwani

Swaka. See under Lala

Swati. See: Swazi

Swazi 3:526

Swina. See: Zezuru

Syan 3:158

Tabi. See: Ingassana

Tabwa 2:636

Tafi. See under Avatime

Tagwana or Tagbana. See under Senufo

Taita 3:116

Tama 2:497

Tamari or Tamberna 2:383

Tambuka. See: Tumbuka

Tambwa. See: Tabwa

Tanga. See: Batanga

Tangale 2:215

Tarok. See: Yergum

Tatoga 3:219

Taturu [Kenya]. See under Dorobo

Taturu [Tanzania]. See: Tatoga

Taveta 3:116

Teda 2:497

Tege. See: Teke

Tegreñña. See: Tigrinya

Teita. See: Taita

Teke (group) 2:510

Tem 2:383

Temne 2:76

Tenda 2:353 [Out of order]

Tera or Terawa 2:215

Teso 3:158

Tete. See: Nyungwe

Tetela 2:629

Teuso. See: $\mathrm{Ik}$

Thagicu 3:116

Tharuka 3:117

Thonga (Tsonga) 3:356 (including

Gwamba); 3:483

Tidn-áal. See: Midob

Tigrai. See: Tigrinya 
Tigre or Tigray 1:634

Tigrino. See: Tigrinya

Tigrinya 1:628

Tikar. See under Nkom

Tikuu (language of Bajuni, dialect of Swahili) 1:644

Tim. See: Tem

Timne. See: Temne

Timu. See: Tem

Tiokossi. See: Anufo

Tirma. See under Mekan

Tiv 2:215

Tabote. See under Bassari [Togo]

Togoyo. See under Feroge

Toma. See: Loma

Tonga (GiTonga) 3:308. 3:357. See also under Tumbuka

Toposa or Topotha 1:572

Torobo. See: Dorobo

Touareg. See: Tuareg

Toura. See: Tura

Tsamba. See: Kasele

Tshi. See: Twi

Tshokwe. See: Chokwe

Tsogap. See: Bagam

Tsogo 2:506

Tsokwe. See: Chokwe

Tsonga. See: Thonga (Tsonga)

Tswa 3:357

Tswana 3:521. See also Sotho-Tswana

Tu Bedawiye. See: Bedawiye

Tuareg 1:510, 2:329

Tuba 2:620

Tubu. See under Teda

Tula 2:216

Tumale. See under Koalib-Tagoi

Tumbuka 3:342 (including Tonga)

Tunen (language of Banen) 2:417

Tura 2:353 [Out of order]

Turkana 3:117

Turumbu. See: Olombo

Twa 2:567

Twareg. See: Tuareg

Twi 2:118. See also Akan; Fante

Tyaman. See: Ebrie

Tyembara. See under Senufo

Tyokosi. See: Anufo

Uallamo. See: Wolamo
Uli. See: Wori

Uluragoli. See: Logoli

Unguja. See under Swahili

Uollamo. See: Wolamo

Urhobo. Indexed as Sobo

Uri. See: Wori

Ushi. See: Aushi

Utchokwe. See: Chokwe

Vai or Vei 2:430

Venda 3:483

Vili 2:510

Vira. See: Bira

Voltaic. See: Gun

Vumba [Tanzania]. See under Swahili

Vute 2:417

Walamo. See: Wolamo

Wandala. See: Mandara

Welaita or Welamo. See: Wolamo

Wisa. See: Bisa [Zambia]

Wolamo. See under Ometo

Wolof 2:3 17

Wori or Wouri or Wuri 2:417

Wute. See: Vute

Xamir. See: Khamir

Xhosa 3:499

Xona. See: Shona

Yaghwatadaxa. See under Mandara

Yaka 2:620

Yalulema. See under Kele [Zaïre]

Yansi 2:620

Yao 3:342, 3:357

Yaunde. See: Ewondo

Yawotatakha. See: Yaghwatadaxa

Yebu. See under Yoruba

Yei. See under Yansi

Yela 2:594

Yemsa. See: Janjero

Yergum 2:216

Yimba. See: Limba

Yombe 2:620

Yoruba 2:246. See also Nago

Yulu or Youlou. See under

Bongo-Baka

Zabarma or Zabrama. See: Dyerma 
Index of Languages cvii

Zande 1:593, 2:605

Zaramo 3:219

Zarek. See: Jarawa

Zarma. See: Dyerma

Zenaga 2:309

Zerma. See: Dyerma
Zezuru 3:331

Ziba. See: Haya

Zigula or Zigoua or Zigua 3:219

Zinjero. See: Janjero

Zinza 3:220

Zulu 3:489 